\title{
Kyrgyz Republic: Request for an 18-Month Arrangement Under the Exogenous Shocks Facility-Staff Report; Staff Supplement; Press Release on the Executive Board Discussion; and Statement by the Executive Director for Kyrgyz Republic.
}

In the context of the request for an 18-month arrangement under the Exogenous Shocks Facility with the Kyrgyz Republic, the following documents have been released and are included in this package:

- $\quad$ The staff report for an 18-Month Request Under the Exogenous Shocks Facility, prepared by a staff team of the IMF, following discussions that ended on October 30, 2008 with officials of the Kyrgyz Republic on economic policies. Based on information available at the time of these discussions, the staff report was completed on November 26, 2008. The views expressed in the staff report are those of the staff team and do not necessarily reflect the views of the Executive Board of the IMF.

- $\quad$ A staff supplement on the joint World Bank/IMF Debt Sustainability Analysis

- $\quad$ A Press Release summarizing the views of the Executive Board as expressed during its December 10, 2008 discussion of the staff report.

- $\quad$ A statement by the Executive Director for the Kyrgyz Republic

The documents listed below have been or will be separately released.

Letter of Intent sent to the IMF by the authorities of the Kyrgyz Republic*

Economic Program by the authorities of the Kyrgyz Republic*

Technical Memorandum of Understanding*

*Also included in Staff Report.

The policy of publication of staff reports and other documents allows for the deletion of market-sensitive information.

Copies of this report are available to the public from

International Monetary Fund • Publication Services $70019^{\text {th }}$ Street, N.W. • Washington, D.C. 20431

Telephone: (202) 623-7430 • Telefax: (202) 623-7201

E-mail: publications@imf.org Internet: http://www.imf.org

Price: $\$ 18.00$ a copy

International Monetary Fund

Washington, D.C. 

INTERNATIONAL MONETARY FUND

KYRGYZ REPUBLIC

\section{Request for an 18-Month Arrangement Under the Exogenous Shocks Facility}

Prepared by the Middle East and Central Asia Department

(In cooperation with other Departments)

Approved by David Owen and Adnan Mazarei

November 26, 2008

Context: Several exogenous shocks have hit the Kyrgyz economy that undermine macroeconomic stability, erode the gains made in poverty reduction, and create balance of payments difficulties.

Request for support under the high-access component of the Exogenous Shocks Facility: The authorities request an 18-month arrangement for SDR66.6 million (75 percent of quota), equivalent to about $\$ 100$ million. SDR16.65 million (about \$25 million) would become available upon Board approval.

The program: The authorities have designed a set of macroeconomic policies aiming to reduce inflation, sustain growth, and protect the poor. Monetary policy will primarily aim at further reducing inflation, while taking into account the slowdown in economic growth. Fiscal policy will similarly continue to strike a balance between supporting monetary policy and protecting growth and the poor.

Discussions: A team comprising Ron van Rooden (head); Veronica Bacalu, Dima Jardaneh, Anna Bordon (all MCD) and Yi Wu (SPR), and assisted by James McHugh (Resident Representative) visited Bishkek during July 22-August 3 and October 2230, 2008. The team met with Prime Minister Chudinov, Minister of Finance Kalimbetova, Minister of Trade and Economic Development Japarov, Minister of Labor and Social Protection Abdullaeva, National Bank Chairman Alapaev, other senior officials, and representatives of the donor community and civil society.

The exchange rate regime is a managed float. The Kyrgyz Republic has accepted the obligations under Article VIII, Sections 2, 3, and 4, and maintains an exchange system free of restrictions on payments and transfers for current international transactions.

Statistics are adequate for surveillance and program monitoring, although they could be improved in some areas (Annex VI). The Kyrgyz Republic subscribes to the SDDS. 


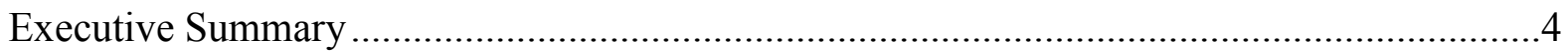

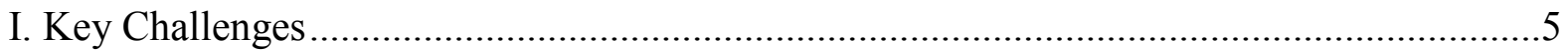

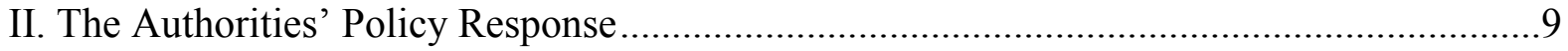

A. Macroeconomic Framework .................................................................. 9

B. Reducing Inflation, Sustaining Growth, and Protecting the Poor ..........................10

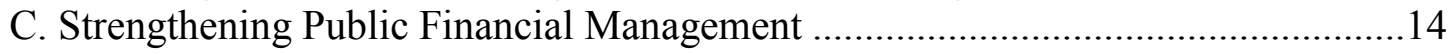

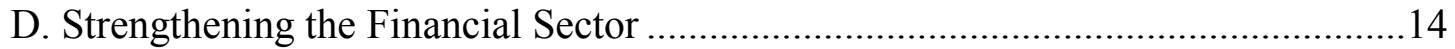

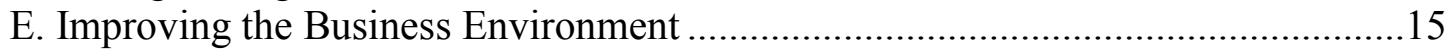

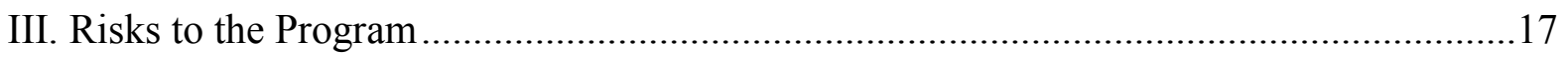

IV. Program Access, Monitoring, and Capacity to Repay the Fund....................................17

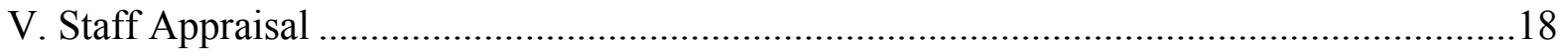

Tables

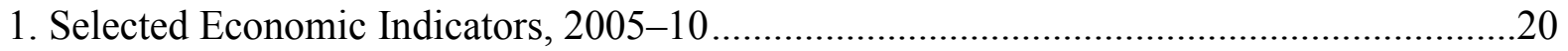

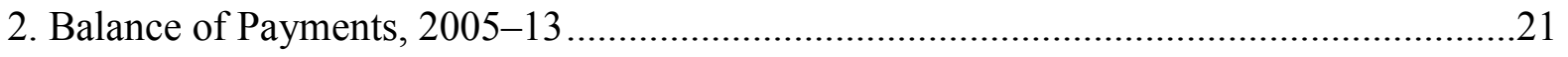

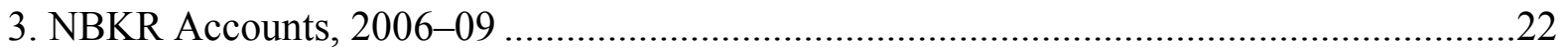

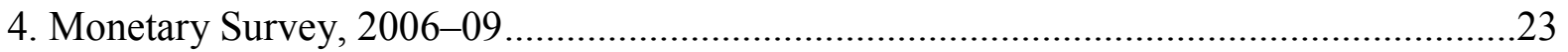

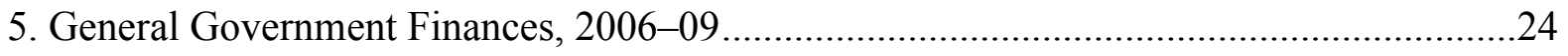

6. General Government Finances, 2006-09.................................................................25

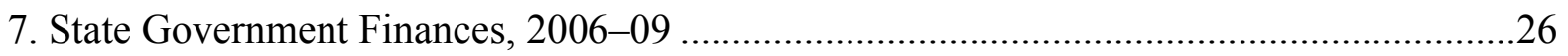

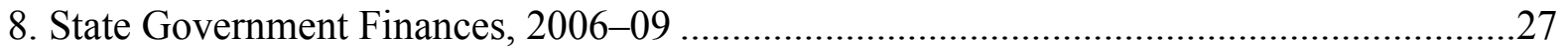

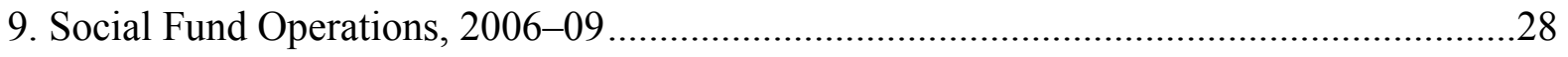

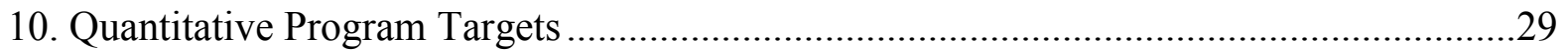

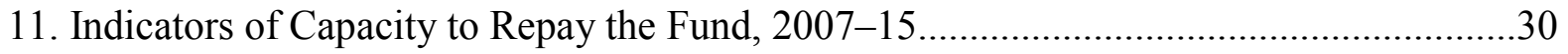

12. Reviews and Disbursements Under the 18-month ESF Arrangement.............................31

Figures

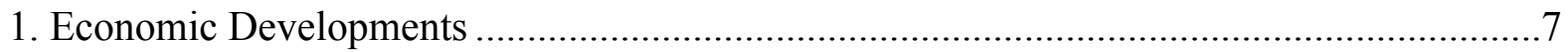

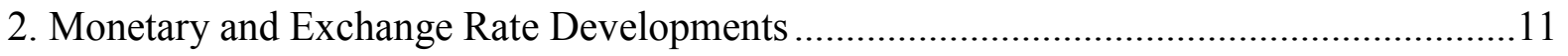

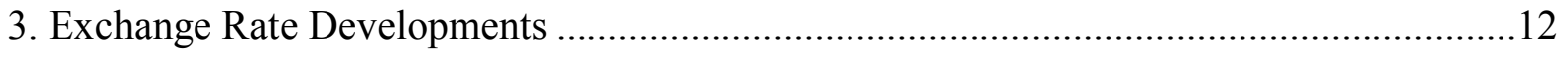

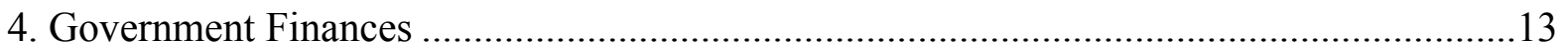

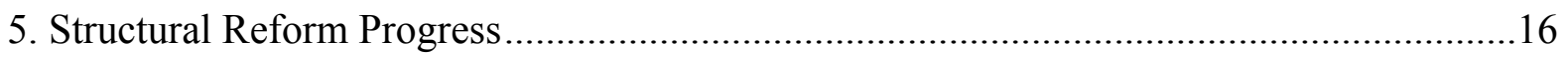


Attachments

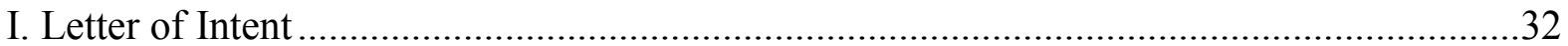

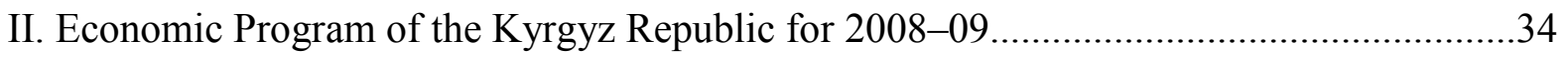

III. Technical Memorandum of Understanding .................................................................48 


\section{EXECUTIVE SUMMARY}

The Kyrgyz economy has been hit by a number of exogenous and largely temporary shocks. International commodity prices continued to rise through much of 2008 , resulting in a surge in inflation to over 30 percent by mid-year and a widening of the current account deficit. A major shortfall in domestic hydropower capacity due to low water levels is causing power shortages and necessitates the import of larger volumes of fuel and electricity, increasing the current account deficit further. In 2008, the current account deficit is projected to widen to $6 \frac{1}{2}$ percent of GDP, compared to a near-balance in 2007. Global and regional economic growth continues to fall in the wake of the international financial crisis. Spillovers from banking sector difficulties in Kazakhstan have resulted in a sharp slowdown in credit expansion in the Kyrgyz Republic. As a result of the combined shocks, growth (excluding gold production) in the Kyrgyz Republic is slowing down as well, from almost 9 percent in 2007 to 5 percent in 2008 and 3 percent in 2009. And with exports and workers' remittances projected to weaken, the current account balance will widen even further in 2009, despite the recent decline in commodity prices, to $8 \frac{1}{2}$ percent of GDP. Part of the gains in poverty reduction achieved in the last 5-10 years may have already been reversed.

The authorities' economic program for 2008-09 aims to address the consequences of the exogenous shocks and to manage the effects on the Kyrgyz economy of the slowdown in regional growth and spillovers from the global financial crisis. The program focuses on reducing inflation, sustaining growth, and protecting the poor. The authorities request an 18-month arrangement under the high-access component of the Exogenous Shocks Facility in the amount of SDR 66.6 million (75 percent of quota) to help achieve these objectives and maintain an adequate level of reserves.

Macroeconomic policies will have to walk a fine line between reducing inflation and supporting growth. Monetary policy has been tightened substantially, reflected in a sharp increase in interest rates this summer, and aims to bring down inflation to close to 10 percent by end-2009. But as inflation falls, monetary policy could be eased gradually to aid the slowing economy. Fiscal policy will similarly aim to balance the need to help reduce inflation with the need to support growth and provide targeted protection to the poor. The budget deficit will widen from 0.3 percent of GDP in 2007 to 1.3 percent of GDP this year, and further to 1.7 percent of GDP in 2009.

Staff supports the authorities' request for an 18-month arrangement under the ESF. There are risks to the program, but in light of the strength of the authorities' policies and their commitment to take additional measures if needed to achieve the program's objectives, the program has a good chance of success. 


\section{Key Challenges}

1. In the last few years, sound economic policies have contributed to strong economic performance. Economic growth accelerated to over 8 percent in 2007. Excluding gold production, it reached almost 9 percent. Inflation remained low until late 2007. Fiscal performance was also impressive, with revenues rising sharply. With firm fiscal policies, and aided by the economic recovery, a gradual appreciation of the som, and Paris Club support, the country was able to achieve a major improvement in debt indicators. Living standards improved as well and poverty rates fell from 54 percent in 2002 to 35 percent in 2007, while extreme poverty dropped from 23 percent to 7 percent.

\section{Since late 2007, a number of exogenous shocks have hit the Kyrgyz economy.}

Some of these shocks were already evident at the time of the final review under the Kyrgyz Republic's last PRGF arrangement in May this year-leading the authorities to request an augmentation of resources by 10 percent of quota (SDR 8.88 million) - but they have since intensified. The shocks include:

- The rise in international food and fuel prices. From mid-2007 to mid-2008, international commodity prices rose by over 50 percent. While commodity prices have fallen in recent months, prices for food and key energy imports (notably natural gas) are expected to remain higher than anticipated in early $2008 .^{1}$

- The slowdown in global and regional economic growth in the wake of the financial turbulence that began in 2007 and intensified this summer. Growth in the region has fallen, especially in Russia and Kazakhstan, and is projected to decline further in 2009. Russia and Kazakhstan accounted for about 40 percent of the Kyrgyz Republic's exports in 2007 and are the main sources of workers' remittances.

- Banking sector difficulties in Kazakhstan. Nearly half of the Kyrgyz banking system is Kazakh-owned. Funding of these banks by their parent institutions has dried up. As a result, credit growth slowed sharply to 27 percent in the 12 months through September 2008, down from 110 percent a year before. No major outflows have occurred yet, but some $\$ 150$ million in liabilities of (mainly the Kazakh-owned) banks to foreign (parent) banks mature in the next 12 months.

- A major shortfall in hydropower capacity. The water level in the Toktogul reservoir, which provides about 90 percent of the country's electricity production, did not recover this summer from a higher-than-normal usage during last year's particularly harsh winter. As a result, the water level is insufficient to ensure adequate power generation during

\footnotetext{
${ }^{1}$ The import price of natural gas was raised by 45 percent effective January 1, 2008 and is expected to be raised by an additional 100 percent effective January 1, 2009.
} 
the coming winter. To avoid an energy crisis, hydropower production will need to be supplemented by generating electricity and heating at two power plants using imported fossil fuels and by importing electricity. An energy conservation plan has been introduced to save water, including voltage reductions, rolling power outages, and temporary closures of schools and government buildings.

- An earthquake hit a remote area along the border with China and Tajikistan in October. Although the impact on the overall economy is small, the earthquake resulted in substantial loss of life and damage to housing and infrastructure in the Nura region.

\section{These exogenous shocks undermine macroeconomic stability, erode living standards, and create balance of payments difficulties:}

- The higher world prices for food and fuel led to a surge in inflation. As the authorities continued to allow full pass-through of international price movements, 12-month inflation reached $32 \frac{1}{2}$ percent in July 2008, up from 51/2 percent in June 2007. Large government wage increases in 2007 and at the start of 2008 are likely to have further contributed to price pressures. The higher food and fuel prices have been feeding through into the prices of other goods and services, including via higher transportation and production costs, with inflation excluding food and fuel products rising to $16^{1 / 4}$ percent in October. Headline inflation eased to $16 \frac{1}{2}$ percent in October, following the recent decline in international commodity prices, and is expected to end 2008 at close to 20 percent.

- The global and regional slowdown, combined with weaker domestic demand due to the high rate of inflation and the sharp drop in credit expansion, is already affecting economic activity. In the first nine months of 2008, non-gold growth (relative to the same period last year) fell to 5.7 percent, down from a growth rate of 9.2 percent in the same period of 2007. As external demand continues to weaken and reflecting the impact of the energy shortages, non-gold growth is expected to slow to 5 percent in 2008 . The overall rate of growth is expected to be about $7 \frac{1}{2}$ percent this year, on account of a strong recovery in gold production.

- The current account balance is worsening substantially in 2008. Due to higher prices alone, the (net) annual cost of importing food and energy is projected to be almost $\$ 300$ million (6 percent of GDP) higher in 2008 than in 2007. In addition, higher import volumes of fuel and electricity will be needed to minimize disruptions in the electricity and heating supply this winter. The authorities estimate the additional costs of fuel imports, along with essential repairs at the two relatively old thermal plants, at $\$ 60$ million. Higher gold export earnings will provide only some compensation, as part of these earnings will leave the country via repatriation of profits and repayments of intracompany loans. Overall, the current account deficit is projected to widen by more than 6 percent of GDP this year. There is a risk that without additional external support official foreign exchange reserves could fall below 3 months of imports. 


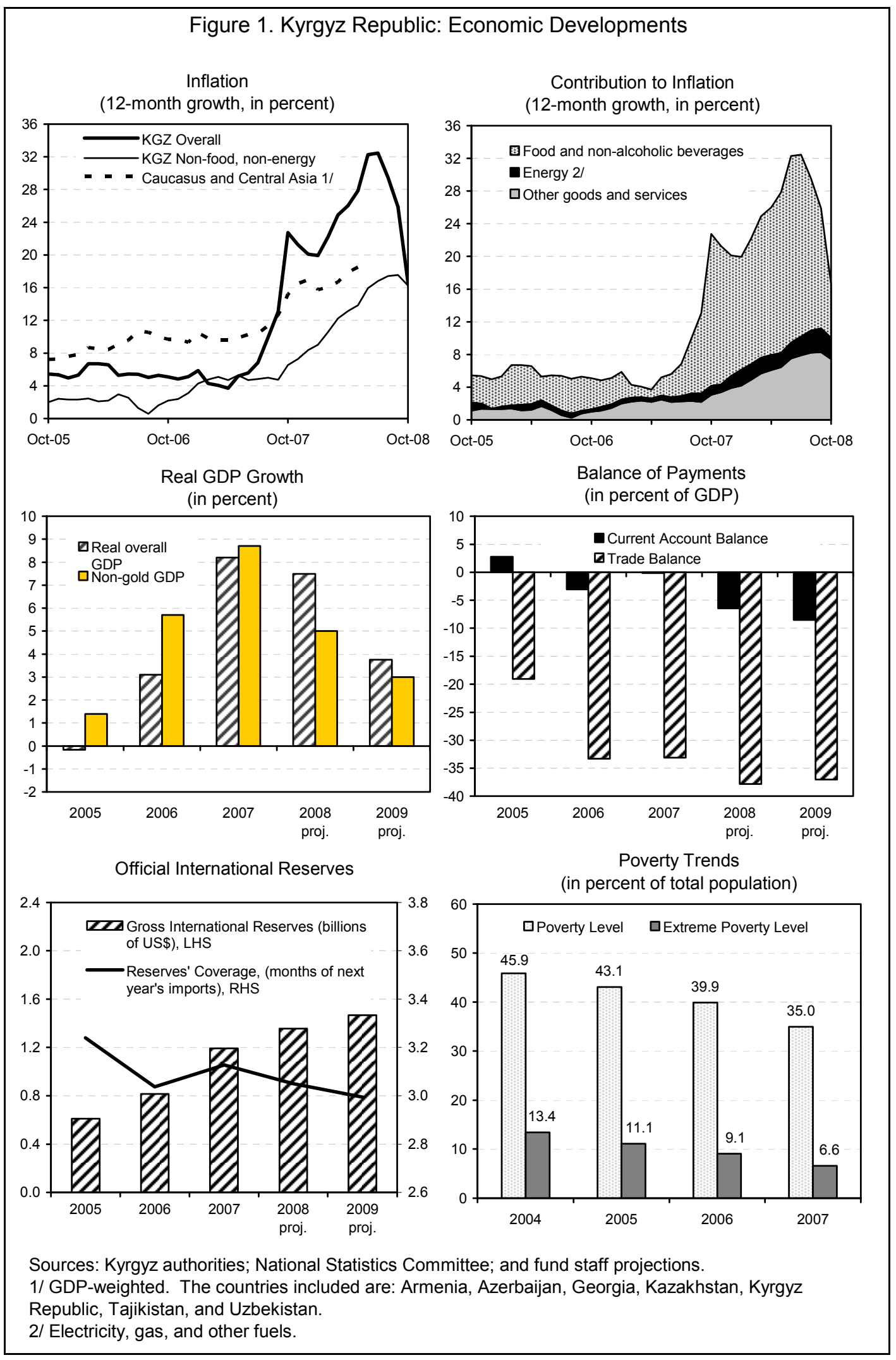


- The food and energy price shocks have particularly hit low-income households. Part of the gains in poverty reduction achieved in the last 5-10 years may have already been reversed. The outlook for the coming winter is worrisome, as energy shortages will affect living conditions. Basic food items and energy are no longer affordable for many low-income households, requiring higher levels of social assistance.

- The commodity price shock, the shortfall in hydropower, and the earthquake create additional pressures on the budget equivalent to over 2 percent of GDP. Already, higher outlays were needed to replenish wheat and fuel reserves and to support state-owned enterprises, mainly in the energy sector, to help cover higher input costs. Additional spending needs for the remainder of 2008 and 2009 include the purchase of fuel and electricity, increased levels of pensions and social support, and relief and reconstruction efforts.

4. These shocks appear to a large extent temporary in nature, calling for increased donor assistance, although adjustment policies and improved regional cooperation on the management of water and energy resources are needed as well. The United Nations system is closely monitoring the food and energy security situation in the region and is coordinating with other donors. Additional donor support is forthcoming, notably from the World Bank, the European Union, and the Asian Development Bank (Box 1). Improved regional cooperation on water and energy issues is a major political challenge.

\section{Box 1. Food and Energy Security: the Donor Response}

The surge in food and fuel prices and growing energy supply difficulties prompted a rapid and coordinated response from the donor community. So far, and in addition to existing projects and programs:

- $\quad$ The World Bank is providing a $\$ 10$ million grant to raise social benefits and increase productivity in the agricultural sector. It will also provide \$11 million as part grant, part concessional loan this winter to help finance additional fuel imports.

- $\quad$ The EU will provide a $\$ 7$ million grant in 2009 to supplement social benefits and may provide further support, including under a planned agricultural support facility.

- $\quad$ The ADB will be providing an additional US\$5 million grant to the budget in 2008 .

- $\quad$ A number of other donors, including the EBRD, USAID, the FAO, and various UN organizations, are providing numerous smaller grants or in-kind assistance to support the agricultural and energy sectors. 


\section{The Authorities’' Policy Response}

5. The authorities have prepared an economic program for 2008-09 to address the consequences of the exogenous shocks and manage the effects on the Kyrgyz economy of the slowdown in regional growth and spillovers from the global financial crisis. The program aims to:

- $\quad$ Reduce inflation to close to 10 percent by end-2009;

- $\quad$ sustain and accelerate economic growth; and

- $\quad$ protect the poor.

The program relies mainly on macroeconomic policies to achieve its goals, but also includes a number of structural measures to support these policies, focusing on strengthening economic management.

6. The authorities' economic program will support implementation of their Country Development Strategy (CDS). The CDS for 2007-10 was discussed by the Fund and Bank Boards in May 2007 and aims at boosting economic efficiency, improving governance and public service delivery, and better targeting of social assistance. An update of the CDS, taking into account the changed international environment, is under preparation.

7. The authorities request the Fund's support under the Exogenous Shocks Facility to help achieve their economic program's objectives and cover the country's balance of payments needs. They request an 18-month arrangement under the high-access component of the ESF, in the amount of SDR 66.6 million (75 percent of quota).

\section{A. Macroeconomic Framework}

8. The program is based on a macroeconomic framework consistent with a further significant reduction in inflation, while attempting to limit the decline in economic growth. Monetary policy will aim to reduce inflation to about 20 percent by end-2008 and to close to 10 percent by end-2009, with a view to returning to single-digit inflation in 2010 . Fiscal policy will support monetary policy, but will balance this with the need to support growth. Non-gold growth is nonetheless projected to slow to 3 percent in 2009 , broadly in line with regional developments. As gold production is expected to rise further, overall growth would reach almost 4 percent. The current account deficit is projected to widen further in 2009, to $81 / 2$ percent of GDP, despite the decline in oil prices and some further rise in gold exports, as exports and workers' remittances are expected to slow due to the regional slowdown and the price of imported gas is raised. Natural gas imports will be about $\$ 130$ million (21/2 percent of GDP) higher in 2009 than in 2008 due to higher prices. 


\section{B. Reducing Inflation, Sustaining Growth, and Protecting the Poor}

\section{Monetary and Exchange Rate Policy}

9. Central to the authorities' policies to reduce inflation has been a tightening of monetary policy - including a sharp increase in interest rates - to fight second-round effects of higher food and fuel prices and to anchor inflation expectations. Since endMarch 2008, interest rates on 3-month Treasury bills have risen by almost 11 percentage points, to $18 \frac{1}{2}$ percent by end-October, exceeding both headline and core inflation. At the same time, the NBKR has pursued an asymmetric intervention policy in the foreign exchange market. Given the high import content of the consumer basket, the NBKR supported the som through unsterilized foreign exchange sales when it came under pressure to depreciate in early 2008 and again in recent months, to limit additional inflationary pressures. When foreign exchange inflows resumed during the summer months, the NBKR allowed the som to appreciate. The NBKR also took advantage of the resumption of inflows to replenish its foreign exchange reserves, but actively sterilized the effects on the money supply by issuing central bank paper. As a result, reserve money growth grew by only $11 \frac{1}{2}$ percent in the first ten months of 2008 .

10. In the period ahead, the NBKR will allow the exchange rate to adjust to the changing economic environment, while continuing to smooth sharp and sudden exchange rate movements. The som has depreciated by almost 13 percent vis-à-vis the U.S. dollar during the last two months. The NBKR will aim to slow the rate of depreciation to limit renewed inflationary pressures and to avoid a disorderly process that could undermine confidence in the som and reverse the decline in dollarization (the program's international reserves targets provide a margin to pursue this policy). The NBKR, however, will avoid large reserve losses if downward pressures on the som persist or intensify, but would further raise interest rates. If foreign exchange inflows resume, the NBKR will allow the som to strengthen, to help bring down inflation faster, while using the opportunity to build up its reserve position. In that case, and provided that inflation continues on a downward path, monetary policy could be eased gradually to support the slowing economy.

11. The government acknowledges the need to support the central bank. The NBKR and ministry of finance are reinforcing coordination, particularly with respect to domestic debt issuance and liquidity management. Also, to avoid monetary policy being constrained by income considerations, the NBKR's capital and income position will be strengthened. 


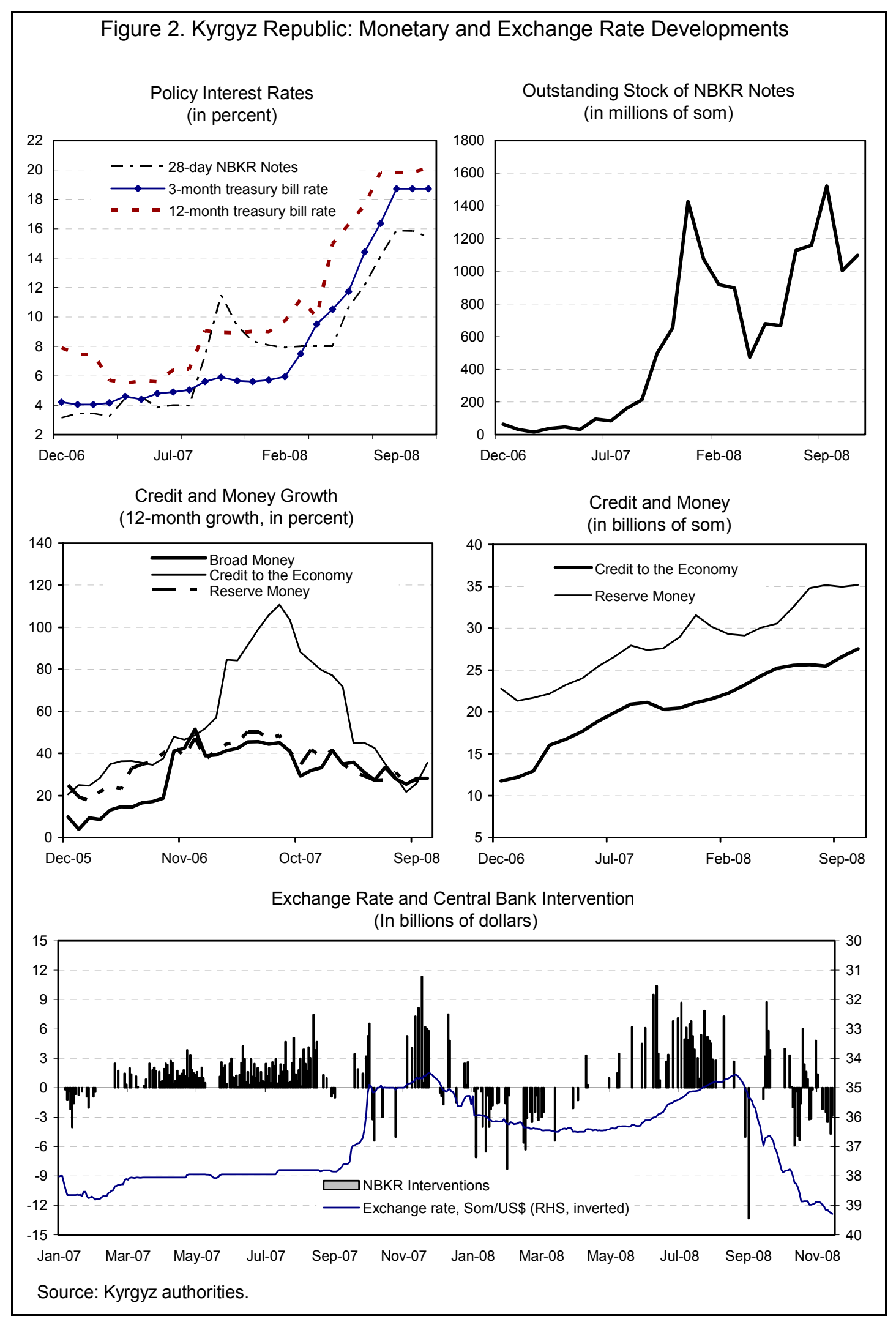




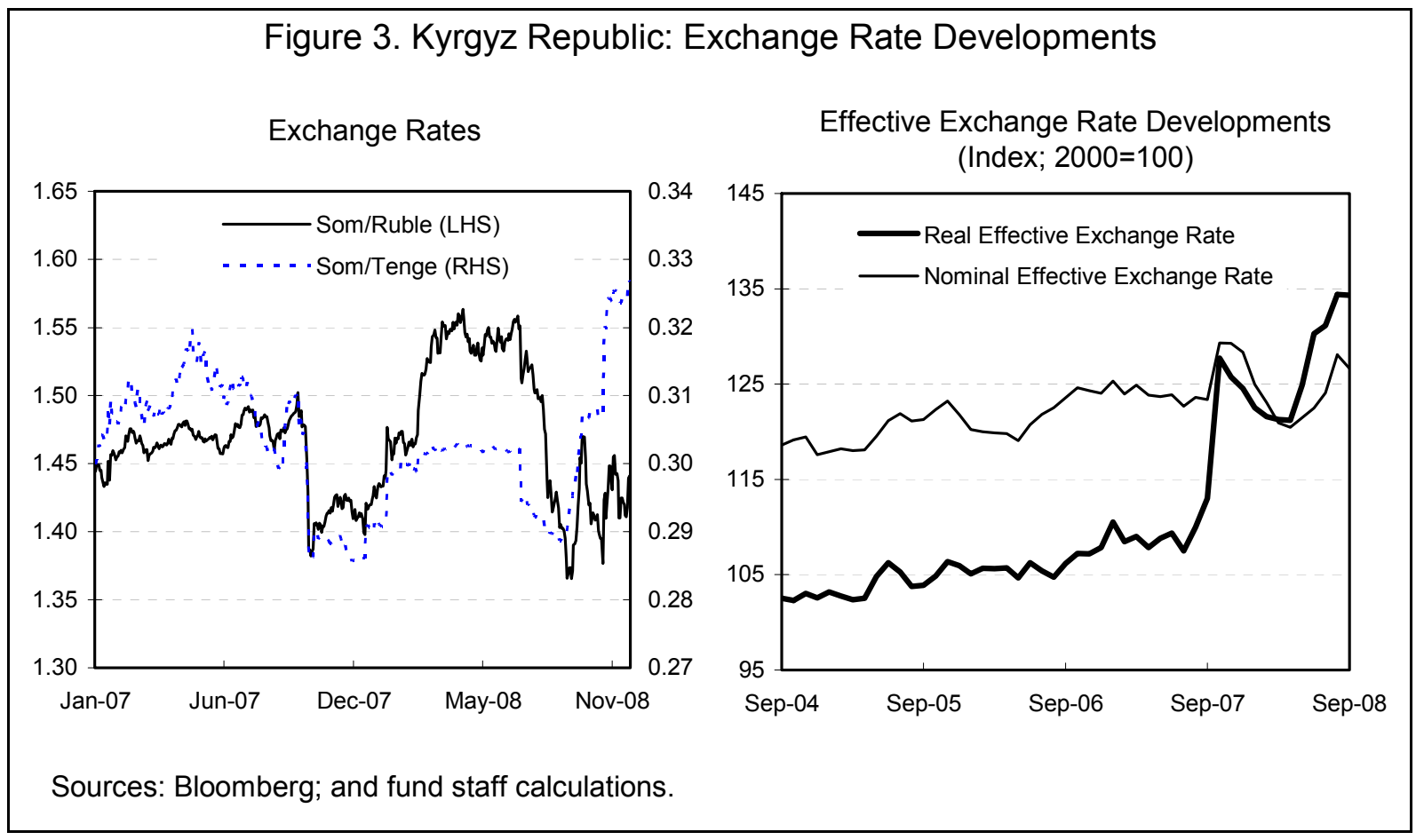

\section{Fiscal Policy}

\section{Fiscal policy will continue to strike a balance between supporting monetary} policy in reducing inflation and protecting growth and the poor, while continuing to reduce the debt burden. Despite the increased spending pressures, fiscal performance in the first nine months of 2008 has been relatively strong, largely owing to a better-than-expected revenue collection, but also to cuts in non-priority spending. With substantial spending needs in the last quarter, the authorities aim to limit the 2008 general government budget deficit to 1.3 percent of GDP, up from a deficit of 0.3 percent of GDP in 2007. The 2009 budget deficit will increase somewhat further, to 1.7 percent of GDP, with sizable additional spending cuts (partly possible because large one-off outlays in 2008 to the energy and agricultural sectors, amounting to about $1 \frac{1 / 2}{2}$ percent of GDP, will not be repeated) offsetting net revenue losses resulting from the introduction of a new tax code. The latter includes a reduction in the VAT rate from 20 percent to 12 percent, to align the rate with those in neighboring countries. This is partially compensated by the introduction of a property tax and a wider turnover tax (which replaces the existing sales, road, and emergency taxes). To help control the wage bill, a civil service reform program will be developed with the help of the World Bank, focusing on payroll policies and establishing a centralized payroll database. Wage increases in 2009 will remain below the projected rate of inflation. To improve the financial sustainability of the pension system, the parliament reversed an earlier decision to reduce the pension retirement age. The authorities will also take the first steps in 2009 towards establishing a 
funded pension pillar. With firm fiscal policies, public debt indicators will continue to improve in 2008-09 and over the longer term.

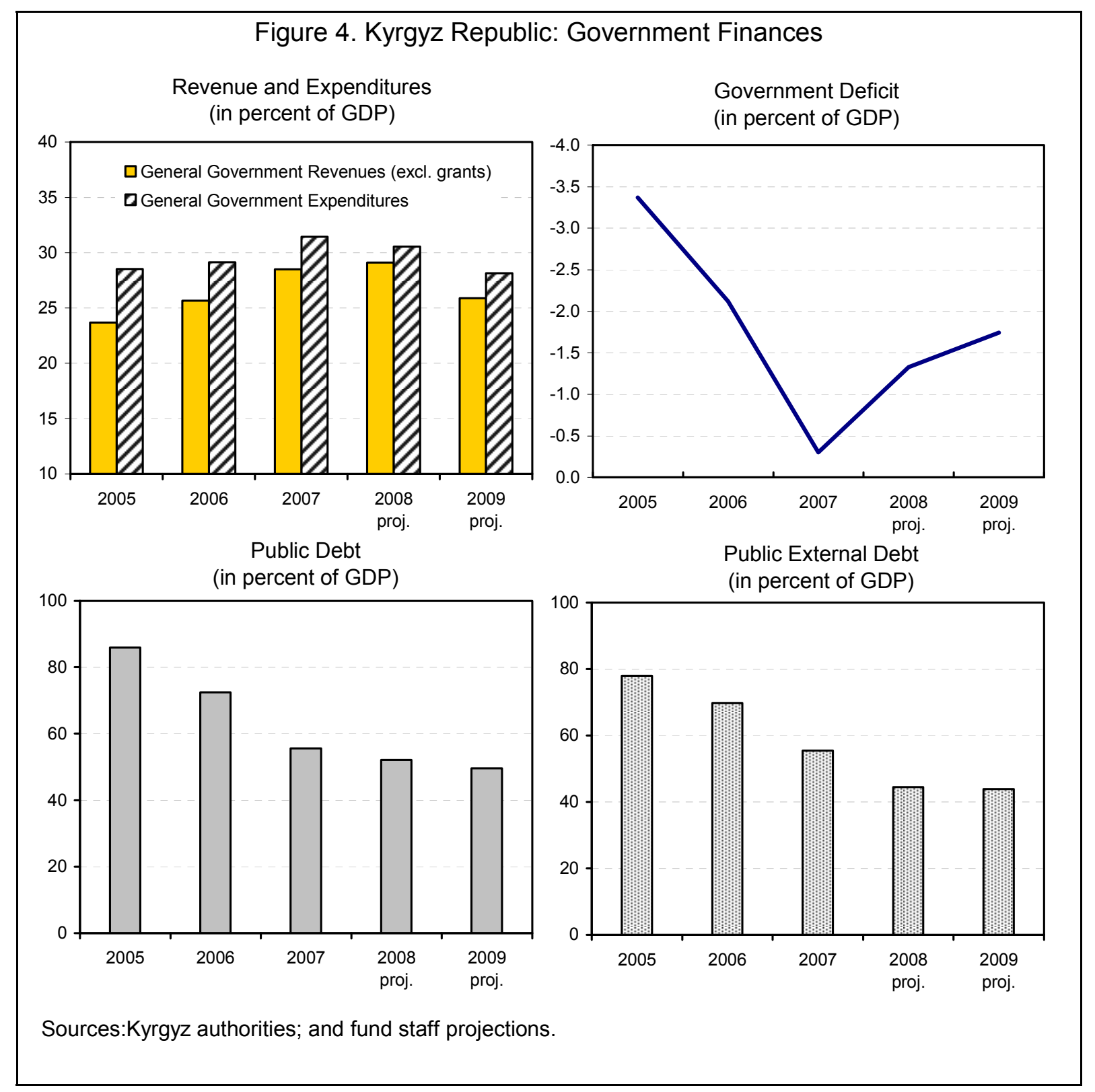

13. Assistance to the poor and vulnerable is being increased. In October 2008, the main targeted social benefit, the Unified Monthly Benefit (UMB), was raised from an average of approximately US\$3 to US\$4 per month per beneficiary. Initially, this increase will be financed from donor grants, but once these run out, the authorities will maintain the higher benefit levels. The authorities will develop criteria to further improve targeting of the UMB, mainly aimed at reducing the exclusion risks, with pilots starting in 2009 and full implementation in 2010. Pensions were also raised in October, broadly offsetting the increase in prices so far in 2008. In addition, VAT rates on grain and flour have been temporarily 
reduced and outlays for specific support programs, such as those providing food to vulnerable children, have been increased.

\section{Strengthening Public Financial Management}

14. The authorities are pressing ahead with public financial management reformswith strong donor support - to ensure greater accountability and transparency. A Treasury modernization program is underway, including increased computerization and the adoption of a new chart of accounts, which will help improve fiscal accounting and reporting. The authorities will also ensure transparency of the Development Fund and the KazakhKyrgyz Fund that are being established to aid the country's longer-term development. The use of public resources for these initiatives will be reflected transparently in the State budget. Moreover, the Development Fund will refrain incurring liabilities that could fall upon the State budget. The 2009 State budget will also include a consolidated presentation of general government finances (including the Social Fund budget) to enable parliament to make a comprehensive assessment of fiscal policy. The authorities will furthermore improve the functioning of the market for government securities, aimed at increasing its liquidity, mainly by reducing the number of treasury bill issues, as domestic capital markets will need to play a greater role in financing the budget deficit.

\section{Strengthening the Financial Sector}

\section{The authorities recognize there are risks and vulnerabilities that could pose} challenges to the Kyrgyz financial system against the backdrop of the global financial crisis. The Kyrgyz banking system has so far weathered the spillover effects from the financial sector difficulties in Kazakhstan relatively well, due to its high capitalization and liquidity. However, the system is not immune to further banking sector difficulties in Russia and Kazakhstan. There is a heightened risk of capital outflows as Kazakh-owned banks will need to roll over maturing obligations to their parent institutions. Also, banks' asset quality may fall with the slowdown in economic activity, especially following last year's rapid credit expansion. Continued vigilance will remain needed to detect any potential liquidity or solvency problems early on. The authorities are prepared to deal with potential banking sector problems, with the NBKR to provide liquidity support and the government to provide capital to systemic banks, should this become necessary. The NBKR will further strengthen its banking supervision, including by conducting more risk-based analyses, engaging in closer oversight over foreign banks, and improving its early-warning system. 
Kyrgyz Republic: Selected Banking System Prudential Indicators, 2005-08

\begin{tabular}{|c|c|c|c|c|c|c|c|c|c|}
\hline & \multirow[t]{2}{*}{2005} & \multirow[t]{2}{*}{2006} & \multicolumn{4}{|c|}{2007} & \multicolumn{3}{|c|}{2008} \\
\hline & & & Q1 & Q2 & Q3 & Q4 & Q1 & Q2 & Q3 \\
\hline & \multicolumn{9}{|c|}{ (in percent) } \\
\hline \multicolumn{10}{|l|}{ Capital adequacy } \\
\hline Regulatory capital/risk-weighted assets & 26.5 & 28.5 & 28.9 & 26.5 & 26.7 & 31.0 & 30.3 & 28.8 & 30.8 \\
\hline Tier 1 capital/risk-weighted assets & 20.6 & 21.7 & 25.2 & 21.7 & 20.7 & 23.6 & 27.6 & 25.2 & 25.7 \\
\hline \multicolumn{10}{|l|}{ Liquidity } \\
\hline Liquidity ratio & 93.3 & 77.8 & 73.6 & 67.8 & 68.9 & 71.3 & 78.0 & 77.1 & 81.0 \\
\hline \multicolumn{10}{|l|}{ Asset quality } \\
\hline Non-performing loans/total loans & 7.7 & 6.2 & 5.1 & 4.2 & 3.5 & 3.6 & 3.6 & 3.9 & 4.1 \\
\hline Loan-loss provisioning/non-performing loans & 49.1 & 51.3 & 56.0 & 55.6 & 57.7 & 59.0 & 61.3 & 56.9 & 56.7 \\
\hline \multicolumn{10}{|l|}{ Profitablitity } \\
\hline Return on equity & 19.6 & 23.2 & 18.5 & 24.3 & 27.4 & 27.0 & 17.2 & 18.0 & 20.4 \\
\hline Return on assets & 2.6 & 3.4 & 3.1 & 4.0 & 4.3 & 4.4 & 3.3 & 3.4 & 3.8 \\
\hline
\end{tabular}

Source: National Bank of the Kyrgyz Republic.

16. A higher level of financial intermediation would help economic development and enhance the effectiveness of monetary policy. The level of financial intermediation is still very low: credit to the economy amounts to only 17 percent of GDP and only some 6 percent of the population has a bank account. To bolster confidence in the banking system, the authorities are considering to advance the introduction of the deposit insurance scheme and to increase its coverage. The scheme, which was approved by parliament in August, was originally slated to become operational in 2010. The authorities are also preparing to sell Ayul Bank (the former agricultural bank) once financial market conditions improve, as authorized by parliament this summer. Ayul Bank has a wide branch network and would obtain a full banking license following its sale. This could greatly enhance access to financial services in rural areas. On the lending side, collateral arrangements have been improved recently, allowing for out-of-court procedures to seize collateral. Banks have also been allowed to share credit information with credit bureaus. Property registration procedures will be improved as well.

\section{E. Improving the Business Environment}

17. Although the current emphasis of the authorities' policies is on addressing the impact of the shocks, they continue to advance their broader structural reform agenda to ensure strong growth over the longer term. Considerable progress has been made in 2008 in improving the business environment, earning the Kyrgyz Republic a ranking as one of the top three reformers in this year's World Bank Doing Business Survey. Progress was made in easing business entry by creating a one-stop shop that streamlined and simplified business registration processes, and in strengthening investor protection. A one-stop shop was also established for obtaining construction permits. Further efforts will focus on streamlining administrative procedures for existing businesses (including tax, customs, and trade procedures) and reducing the number of inspections by various agencies. All draft regulations which have a bearing on private businesses will be subjected to a mandatory review process to assess their regulatory impact. 
Figure 5. Kyrgyz Republic: Structural Reform Progress

World Bank Ease of Doing Business, 2008/09 1/

Progress in World Bank Doing Business Ranking 1/
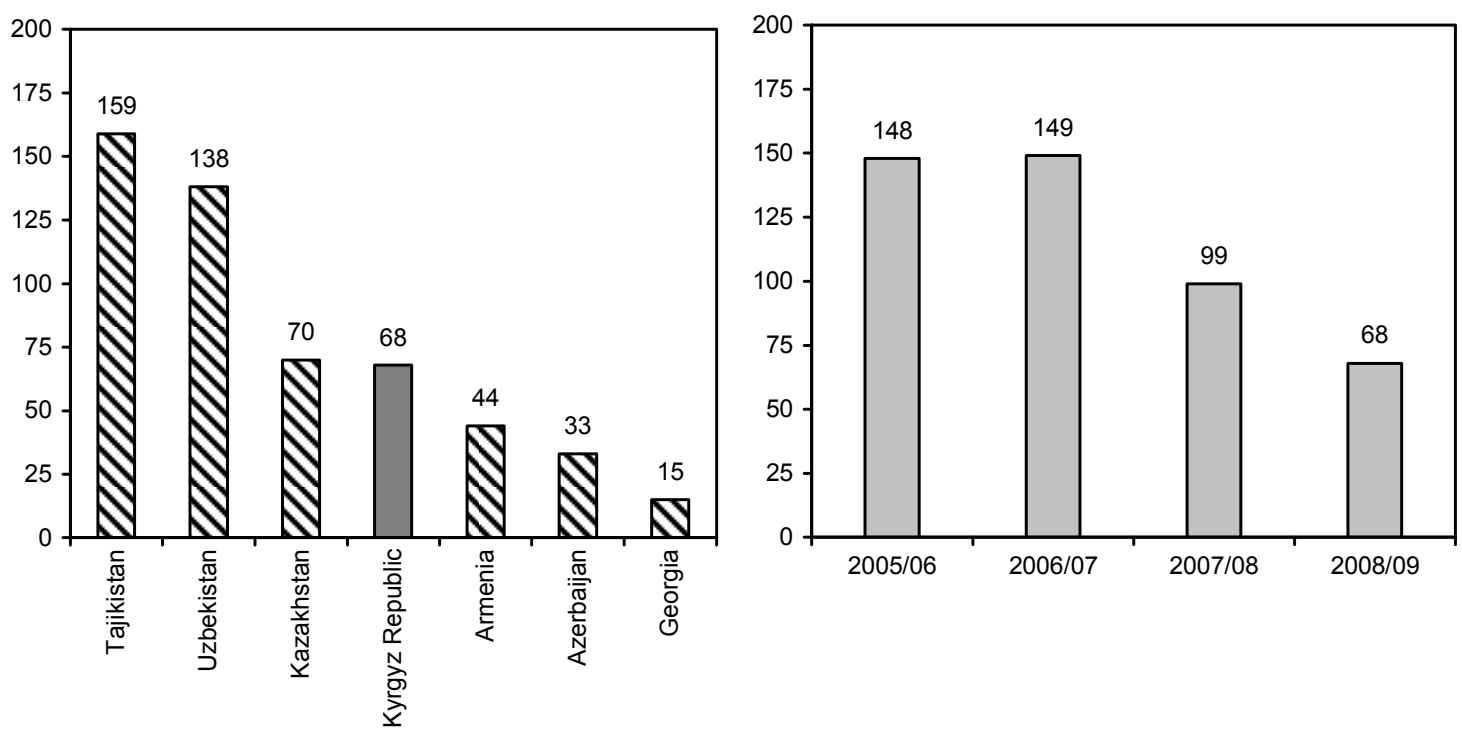

EBRD Transition Indicators, 2008 2/

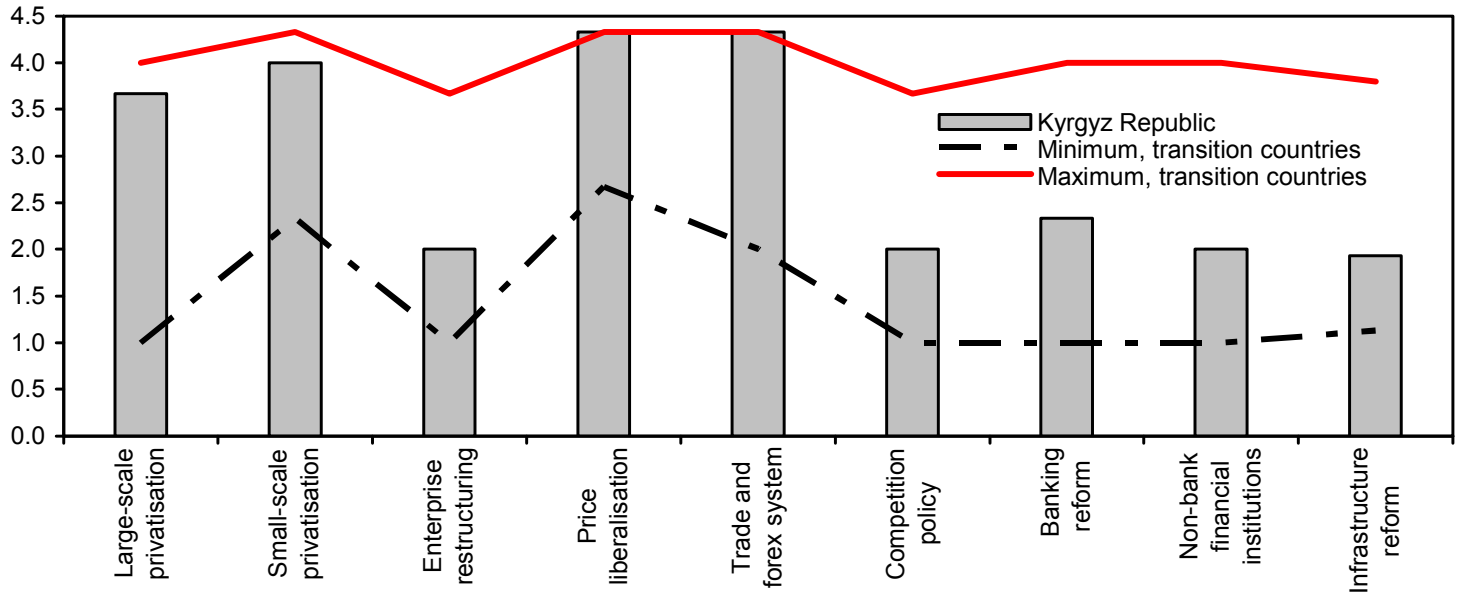

Sources: World Bank; EBRD.

1/ A lower score means greater ease of doing business. Rankings over time are not fully comparable due to coverage and methodology changes.

2/ A higher score means greater progress in transition.

18. The current uncertainty regarding energy security highlights the need to continue with energy sector reforms. The authorities have adopted a National Energy Program. The program aims to improve the energy sector's financial soundness and reduce demand by gradually raising utility tariffs, to make the sector attractive for private investors. A first round of tariff increases was implemented in July this year and a second round is scheduled for mid-2009. Parts of the energy sector (mainly distribution and transmission) 
will be made available for privatization, while private entry will also be allowed in power generation. A feasibility study for the large Kambar-Ata I hydropower project is expected to be completed in the spring of 2009. The authorities are seeking private participation as well as support from international donors for the development of this regionally critical project.

\section{Risks to the Program}

19. The authorities are committed to restoring macroeconomic stability and moving ahead with their reform program, but there are significant downside risks.

- A major economic risk is a further deterioration in the international economic environment, including a more severe regional economic slowdown, worsening financial sector problems in the region, and possibly new international commodity price increases. Risks also arise from the large structural changes to the tax system and the challenges these pose to tax administration. Energy shortages could occur again during the 2009/10 winter season, as addressing the underlying structural problems will take time and require regional cooperation. Additional adjustment efforts will be required if these risks materialize, and the authorities have indicated they are ready to implement additional measures as needed. A further deterioration of the external outlook or renewed energy shortages next year would also warrant stronger donor support.

- $\quad$ Political and social tensions could limit the authorities' ability to implement difficult measures. However, the president's party has a large majority in the parliament and the government should be able to obtain parliament's support to advance its policy agenda.

- Administrative capacity constraints may limit the authorities' ability to implement reforms. Several donors are active to help build capacity, including through sizable technical assistance programs. Supplementing a World Bank project to strengthen capacity in economic management, the Fund will station a resident economic advisor in the ministry of economy and offer two large-scale training courses, to build capacity in macroeconomic analysis and forecasting and enhance coordination among agencies involved in economic policy making. Donor activities are relatively well-coordinated, with key donors organized in the Joint Country Support Strategy, a donor coordination mechanism established to assist the authorities with achieving the objectives of their CDS.

\section{Program Access, Monitoring, And Capacity to Repay the Fund}

20. In light of the sizable exogenous shocks and the balance of payments needs these create, staff supports the authorities' request for an access of 75 percent of quota. The proposed access takes into account the size and nature of the shocks, the strength of the authorities' policies, the country's outstanding use of Fund resources, and the expected additional assistance from other international financial institutions and bilateral donors. The shocks are clearly sizable and to a large extent temporary in nature, warranting a significant role for financing in support of policy adjustment efforts. The proposed Fund support would 
cover a little over half of the projected balance of payments needs of nearly $\$ 185$ million, while other donors are expected to cover the rest. The proposed access would smooth the adjustment and help maintain reserve coverage at just over 3 months of imports. It would also mean that the Fund would provide net support to the country at a time when it is faced with considerable challenges.

21. Conditionality centers on macroeconomic policies. Performance criteria (PCs) and structural benchmarks are set out in Tables 1 and 2 attached to the authorities' letter of intent. Three program reviews are planned, with the first review mission scheduled for March 2009. In light of the uncertainty surrounding developments in the winter months, PCs for end-June and end-December 2009 will be set at the time of the first review. Structural conditionality is derived from the authorities' reform agenda and focuses on fiscal and financial issues that are critical to strengthening their macroeconomic management capacity, including closer coordination, enhanced financial independence of the central bank, improved delineation of responsibilities for supporting systemic banks, and safeguarding access to domestic financing for the budget.

\section{The Kyrgyz Republic has established a record of timely servicing its obligations} to the Fund, and its capacity to repay the Fund is expected to remain strong. The debt sustainability analysis places the Kyrgyz Republic at a moderate risk of debt distress, a major improvement from only a few years ago. With the continuation of sound economic policies, the net present value of public external debt-to-GDP ratio is projected to continue to decline, to below 30 percent in 2008-09. An update of the safeguards assessment of the NBKR has been initiated and will be completed by the time of the first review.

\section{Staff ApPraisal}

23. The Kyrgyz Republic has been hit hard by several exogenous shocks. The impact on the economy is already clearly evident. How much of an effect these shocks have on the poor is as yet unclear, but a large part of the gains achieved in reducing poverty over the last several years may have already been reversed.

\section{The authorities have adopted strong macroeconomic policies to address the} shocks and manage the economy in a much more challenging international environment. These policies are well-balanced, walking a fine line between quickly reducing inflation and protecting growth and the poor. A further tightening of monetary policy may still be needed to firmly reduce inflation expectations, especially if downward pressures on the exchange rate intensify. But if inflation continues on a downward path, a gradual easing of monetary policy would be warranted, particularly if growth slows more than already anticipated. In that case, a looser fiscal stance might also be warranted. A smaller reduction in the VAT rate would have been preferable, as it would have avoided the need to introduce a less efficient turnover tax. 


\section{The emphasis on macroeconomic policies to restore macrostability is}

appropriate. At the same time, it is welcome that the authorities acknowledge that advancing broad-based structural reforms to ensure strong economic growth over the medium and long term will continue to be needed. In this regard, they are to be commended for the substantial progress they have made in implementing reforms this past year. It will be particularly important to move ahead with energy sector reforms to reduce the risk of a repeat of the current difficulties.

26. The banking system has so far been resilient to the shocks, but the NBKR needs to remain vigilant. Strong supervisory efforts continue to be needed to detect and address any banking sector problems that may arise from a potential decline in asset quality or further spillover effects from the international financial crisis. Advancing the introduction of the deposit insurance could help bolster confidence in the banking system.

27. Staff supports the authorities' request for an arrangement under the ESF. The arrangement would help the authorities achieve the objectives of their economic program. It would also provide comfort to other donors, who are increasing their support to the Kyrgyz Republic, that sound economic policies remain in place. There are risks to the program, but in light of the authorities' track record and their commitment to take additional measures if needed to achieve the program's objectives, the new program has a good chance of success. 
Table 1. Kyrgyz Republic: Selected Economic Indicators, 2005-10

\begin{tabular}{|c|c|c|c|c|c|c|}
\hline & 2005 & 2006 & 2007 & 2008 & 2009 & 2010 \\
\hline & & & Prel. & \multicolumn{3}{|c|}{ Projections } \\
\hline Nominal GDP (in billions of soms) & 100.9 & 113.8 & 139.7 & 175.8 & 202.4 & 234.1 \\
\hline Nominal GDP (in millions of U.S. dollars) & 2,460 & 2,837 & 3,748 & 4,805 & 5,124 & $5,838.4$ \\
\hline Real GDP (growth in percent) & -0.2 & 3.1 & 8.2 & 7.5 & 3.7 & 6.2 \\
\hline Non-gold real GDP (growth in percent) & 1.4 & 5.7 & 8.7 & 5.0 & 3.0 & 4.5 \\
\hline GDP per capita (in U.S. dollars) & 478 & 546 & 713 & 906 & 956 & 1,075 \\
\hline Consumer prices (12-month percent change, eop) & 4.9 & 5.1 & 20.1 & 19.8 & 11.0 & 7.0 \\
\hline Consumer prices (12-month percent change, average) & 4.3 & 5.6 & 10.2 & 24.5 & 11.9 & 8.9 \\
\hline Producer prices (12-month percent change, eop) & 6.3 & 10.4 & 20.6 & $\ldots$ & $\ldots$ & $\ldots$ \\
\hline Producer prices (12-month percent change, average) & 2.8 & 15.3 & 11.9 & $\ldots$ & $\ldots$ & $\ldots$ \\
\hline \multicolumn{7}{|l|}{ Investment and savings (in percent of GDP) } \\
\hline Investment & 21.8 & 22.8 & 21.6 & 22.1 & 23.2 & 24.6 \\
\hline Public & 4.8 & 4.3 & 4.8 & 6.5 & 6.9 & 6.5 \\
\hline Private & 17.0 & 18.5 & 16.8 & 15.6 & 16.3 & 18.1 \\
\hline Savings & 24.6 & 19.8 & 21.5 & 15.6 & 14.7 & 18.3 \\
\hline Public & 1.0 & 1.6 & 4.2 & 5.5 & 3.2 & 4.3 \\
\hline Private & 23.6 & 18.2 & 17.3 & 10.1 & 11.5 & 14.0 \\
\hline Savings-investment balance & 2.8 & -3.1 & -0.2 & -6.4 & -8.5 & -6.3 \\
\hline \multicolumn{7}{|l|}{ General government finances (in percent of GDP) $1 /$} \\
\hline Total revenue and grants & 24.7 & 26.4 & 30.8 & 31.7 & 28.5 & 28.7 \\
\hline of which: Tax revenue & 20.0 & 21.4 & 22.9 & 23.9 & 22.2 & 23.0 \\
\hline Total expenditure (including net lending) & 28.1 & 28.9 & 31.5 & 33.1 & 30.2 & 30.2 \\
\hline of which: Current expenditure & 23.7 & 24.8 & 26.6 & 26.2 & 23.5 & 23.9 \\
\hline Capital expenditure & 4.8 & 4.3 & 4.8 & 6.5 & 6.9 & 6.5 \\
\hline Overall fiscal balance & -3.6 & -2.1 & -0.3 & -1.3 & -1.7 & -1.5 \\
\hline Primary balance & -2.0 & -1.2 & 0.3 & -0.5 & -0.9 & -0.7 \\
\hline Total public debt & 85.9 & 72.5 & 57.7 & 52.2 & 47.2 & 45.0 \\
\hline \multicolumn{7}{|l|}{ Banking sector 2/ } \\
\hline Reserve money (percent change, eop) & 24.9 & 47.4 & 38.5 & 17.6 & 15.0 & 19.1 \\
\hline Broad money (percent change, eop) & 9.9 & 51.6 & 33.3 & 19.0 & 17.0 & 21.0 \\
\hline Credit to private sector (percent change, eop) & 20.5 & 48.6 & 79.7 & 25.6 & 21.1 & 24.2 \\
\hline Credit to private sector (in percent of GDP) & 7.8 & 10.4 & 15.1 & 15.8 & 16.6 & 17.8 \\
\hline Velocity of broad money $3 /$ & 4.7 & 3.5 & 3.2 & 3.4 & 3.3 & 3.2 \\
\hline Interest rate $4 /$ & 4.3 & 4.2 & 5.6 & $\ldots$ & $\ldots$ & $\ldots$ \\
\hline \multicolumn{7}{|l|}{ External sector } \\
\hline Current account balance (in percent of GDP) & 2.8 & -3.1 & -0.2 & -6.4 & -8.5 & -6.3 \\
\hline Export of goods and services (million USD) & 1,053 & 1,485 & 2,244 & 3,138 & 3,037 & 3,476 \\
\hline Export growth (percent change) & 2.9 & 40.9 & 51.1 & 39.9 & -3.2 & 14.5 \\
\hline Import of goods and services (million USD) & 1,397 & 2,253 & 3,218 & 4,580 & 4,572 & 5,048 \\
\hline Import growth (percent change) & 23.9 & 61.3 & 42.8 & 42.3 & -0.2 & 10.4 \\
\hline Gross official reserves (million USD) 5/ & 608 & 814 & 1,194 & 1,162 & 1,260 & 1,449 \\
\hline Gross reserves (months of next year imports, eop) & 3.2 & 3.0 & 3.1 & 3.0 & 3.0 & 3.1 \\
\hline Gross reserves to short-term debt ratio (in percent) 6/ & 229.1 & 332.4 & 329.2 & 270.2 & $\ldots$ & $\ldots$ \\
\hline External public debt outstanding (in percent of GDP) & 78.0 & 69.8 & 55.5 & 44.4 & 43.8 & 41.2 \\
\hline External public debt service-to-export ratio (in percent) & 6.5 & 4.4 & 2.9 & 2.3 & 2.8 & 2.7 \\
\hline \multicolumn{7}{|l|}{ Memorandum items } \\
\hline Exchange rate (soms per U.S. dollar, average) & 41.0 & 40.1 & 37.3 & $\ldots$ & $\ldots$ & $\ldots$ \\
\hline Real effective exchange rate $(2000=100)$ (average) & 104.3 & 106.0 & 113.6 & $\ldots$ & $\ldots$ & $\ldots$ \\
\hline
\end{tabular}

Sources: Kyrgyz authorities; and Fund staff estimates and projections.

1/ General government comprises state government and Social Fund finances. State government comprises central and local governments.

2/ At actual exchange rates for 2005-07, projections at program exchange rates (including Som 38.21 per 1 US $\$$ ) for 2008-09.

3/ 12-month GDP over end-period broad money.

4/ Interest rate on 3-month treasury bills.

$5 /$ Gross reserves exclude international reserves of the NBKR that are pledged or blocked.

6/ Short-term debt includes public and private debt amortization with remaining maturity of less than one year in addition to

commercial banks' gross foreign liabilities. 
Table 2. Kyrgyz Republic: Balance of Payments, 2005-13 (In millions of U.S. dollars)

\begin{tabular}{|c|c|c|c|c|c|c|c|c|c|}
\hline & 2005 & 2006 & 2007 & 2008 & 2009 & 2010 & 2011 & 2012 & 2013 \\
\hline & & & Prel. & \multicolumn{6}{|c|}{ Projections } \\
\hline Current account balance & 69 & -87 & -6 & -310 & -438 & -368 & -325 & -268 & -260 \\
\hline excluding transfers & -432 & -817 & $-1,026$ & $-1,583$ & $-1,672$ & $-1,745$ & $-1,835$ & $-1,927$ & $-2,021$ \\
\hline Trade balance & -311 & -686 & $-1,076$ & $-1,654$ & $-1,770$ & $-1,856$ & $-2,047$ & $-2,071$ & $-2,213$ \\
\hline Exports, fob & 794 & 1,106 & 1,559 & 2,162 & 2,014 & 2,280 & 2,475 & 2,825 & 3,087 \\
\hline CIS countries & 413 & 676 & 976 & 1,285 & 1,164 & 1,287 & 1,464 & 1,617 & 1,794 \\
\hline o/w energy products & 33 & 43 & 106 & 283 & 175 & 197 & 217 & 237 & 253 \\
\hline Of which: Re-exports of consumer goods $1 /$ & 107 & 200 & 223 & 256 & 253 & 279 & 319 & 353 & 394 \\
\hline Non-CIS countries & 382 & 430 & 584 & 877 & 851 & 993 & 1,012 & 1,208 & 1,294 \\
\hline of which: Gold & 231 & 206 & 225 & 478 & 493 & 592 & 563 & 720 & 761 \\
\hline of which: Re-exports of oil products & 46 & 106 & 173 & 134 & 91 & 101 & 107 & 112 & 114 \\
\hline Imports, fob & 1,106 & 1,792 & 2,635 & 3,816 & 3,784 & 4,136 & 4,522 & 4,896 & 5,300 \\
\hline CIS countries & 641 & 926 & 1,417 & 2,139 & 2,018 & 2,173 & 2,339 & 2,541 & 2,713 \\
\hline of which: Energy (including for re-exports) & 289 & 460 & 681 & 1,223 & 1,096 & 1,181 & 1,246 & 1,331 & 1,396 \\
\hline Non-CIS countries & 465 & 867 & 1,219 & 1,677 & 1,766 & 1,963 & 2,183 & 2,355 & 2,587 \\
\hline of which: Goods for re-exports & 97 & 182 & 202 & 233 & 230 & 254 & 290 & 321 & 358 \\
\hline Services & -32 & -82 & 102 & 213 & 234 & 284 & 358 & 383 & 449 \\
\hline Receipts & 259 & 379 & 684 & 977 & 1,023 & 1,196 & 1,381 & 1,537 & 1,718 \\
\hline Payments & -291 & -461 & -582 & -764 & -788 & -912 & $-1,023$ & $-1,155$ & $-1,269$ \\
\hline Income & -88 & -48 & -52 & -141 & -137 & -174 & -146 & -238 & -257 \\
\hline Interest payments 2/ & -36 & -20 & -26 & -23 & -33 & -38 & -42 & -47 & -50 \\
\hline Other net income & -52 & -28 & -27 & -118 & -103 & -136 & -104 & -191 & -207 \\
\hline Current Transfers (net) & 500 & 730 & 1,020 & 1,273 & 1,234 & 1,377 & 1,510 & 1,658 & 1,761 \\
\hline of which: Private & 477 & 717 & 990 & 1,227 & 1,198 & 1,342 & 1,476 & 1,623 & 1,725 \\
\hline Capital Account & -13 & -36 & -61 & 49 & 91 & 82 & 89 & 95 & 100 \\
\hline Official & 50 & 37 & 58 & 96 & 116 & 105 & 109 & 111 & 114 \\
\hline Private & -63 & -73 & -120 & -47 & -25 & -23 & -20 & -17 & -14 \\
\hline Financial account & -7 & 313 & 344 & 351 & 387 & 437 & 431 & 376 & 386 \\
\hline Commercial banks & -27 & -44 & 104 & -25 & -25 & 0 & 0 & 0 & 0 \\
\hline Medium-and long-term loans, net & 11 & 83 & 54 & 63 & 67 & 101 & 113 & 29 & 38 \\
\hline Disbursement & 89 & 120 & 105 & 107 & 127 & 204 & 242 & 165 & 184 \\
\hline of which: Loan financed PIP & 79 & 87 & 59 & 55 & 89 & 158 & 187 & 100 & 109 \\
\hline Amortization 2/ & -59 & -37 & -51 & -44 & -60 & -103 & -129 & -136 & -147 \\
\hline FDI and Portfolio investment (net) & 45 & 179 & 190 & 245 & 277 & 277 & 278 & 307 & 308 \\
\hline Net short-term flows, and errors and omissions & -36 & 94 & 41 & 68 & 68 & 60 & 40 & 40 & 40 \\
\hline Overall balance & 49 & 189 & 323 & 90 & 40 & 152 & 195 & 202 & 226 \\
\hline Financing & -49 & -189 & -323 & -128 & -122 & -215 & -195 & -202 & -226 \\
\hline Net international reserves & -93 & -193 & -327 & -128 & -122 & -215 & -195 & -202 & -226 \\
\hline Gross official reserves (- increase) & -81 & -170 & -307 & -121 & -99 & -190 & -171 & -182 & -208 \\
\hline IMF (net) & -12 & -24 & -21 & -7 & -23 & -25 & -24 & -21 & -18 \\
\hline Exceptional Financing (including arrears) & 44 & 4 & 5 & 0 & 0 & 0 & 0 & 0 & 0 \\
\hline Financing gap & $\ldots$ & $\ldots$ & $\ldots$ & 38 & 82 & 63 & 0 & 0 & 0 \\
\hline of which: IMF purchases & $\ldots$ & $\ldots$ & $\ldots$ & 25 & 51 & 26 & 0 & 0 & 0 \\
\hline Other donors & $\ldots$ & $\ldots$ & $\ldots$ & 13 & 31 & 37 & 0 & 0 & 0 \\
\hline \multicolumn{10}{|l|}{ Memorandum items: } \\
\hline GDP (in millions of U.S. dollars) & 2,460 & 2,837 & 3,748 & 4,805 & 5,124 & 5,838 & 6,370 & 6,992 & 7,550 \\
\hline Current account balance (in percent of GDP) & 2.8 & -3.1 & -0.2 & -6.4 & -8.5 & -6.3 & -5.1 & -3.8 & -3.4 \\
\hline Growth of exports of goods and services (volume, percent) & -4.8 & 19.5 & 40.1 & 20.5 & 7.2 & 10.2 & 6.9 & 9.9 & 8.2 \\
\hline Growth of imports of goods and services (volume, percent) & 11.3 & 42.6 & 34.8 & 19.2 & 7.4 & 7.5 & 7.5 & 7.5 & 7.5 \\
\hline Terms of trade (goods, percentage change) & -3.6 & 5.7 & 1.7 & -3.9 & -4.9 & 1.0 & 1.7 & 1.7 & 1.0 \\
\hline External Public Debt (US\$ million) 3/ & 1,918 & 1,982 & 2,079 & 2,135 & 2,246 & 2,404 & 2,527 & 2,566 & 2,616 \\
\hline as percent of GDP & 78.0 & 69.8 & 55.5 & 44.4 & 43.8 & 41.2 & 39.7 & 36.7 & 34.6 \\
\hline External Public Debt (NPV US \$ million) 2/ & 1,145 & 1,257 & 1,372 & 1,410 & 1,495 & 1,601 & 1,693 & 1,741 & 1,799 \\
\hline as percent of GDP & 47 & 44 & 37 & 29 & 29 & 27 & 27 & 25 & 24 \\
\hline as percent of exports & 109 & 85 & 61 & 45 & 49 & 46 & 44 & 40 & 37 \\
\hline Public debt service-to-exports ratio 3 / 4/ & 6.5 & 4.4 & 2.9 & 2.3 & 2.8 & 2.7 & 2.5 & 2.2 & 2.0 \\
\hline Gross reserves $5 /$ & 608 & 814 & 1,194 & 1,162 & 1,260 & 1,449 & 1,621 & 1,802 & 2,011 \\
\hline in months of subsequent year's imports & 3.2 & 3.0 & 3.1 & 3.0 & 3.0 & 3.1 & 3.2 & 3.3 & 3.4 \\
\hline Financing gap as percent of GDP & 0.0 & 0.0 & 0.0 & 0.8 & 1.6 & 1.1 & 0.0 & 0.0 & 0.0 \\
\hline
\end{tabular}

Sources: Kyrgyz authorities and Fund staff estimates and projections.

$1 /$ Reflects adjustments to the official statistics to account for the staff's estimate for re-exports through informal border trade.

2/ Starting 2006, interest and amortization reflect bilateral agreements signed following March 2005 Paris Club agreement to grant debt relief on London terms.

$3 /$ Public and publicly guaranteed debt.

4/ Net of rescheduling.

5/ Valued at end-period exchange rates. The discrepancy between the difference in year-end stocks and the change in reserves under Financing is caused by movements in prices and exchange rates. For years 2008-10, include prospective disbursements from IMF and other donors. 
Table 3. Kyrgyz Republic: NBKR Accounts, 2006-09 1/

\begin{tabular}{|c|c|c|c|c|c|c|c|c|c|c|}
\hline & \multirow{2}{*}{$\begin{array}{r}2006 \\
\text { Dec. } \\
\text { Act. }\end{array}$} & \multirow{2}{*}{$\begin{array}{l}2007 \\
\text { Dec. } \\
\text { Act. }\end{array}$} & \multicolumn{4}{|c|}{2008} & \multicolumn{4}{|c|}{2009} \\
\hline & & & $\begin{array}{l}\text { Mar. } \\
\text { Act. }\end{array}$ & $\begin{array}{l}\text { Jun. } \\
\text { Act. }\end{array}$ & $\begin{array}{r}\text { Sep. } \\
\text { Act. }\end{array}$ & $\begin{array}{l}\text { Dec. } \\
\text { Proj. }\end{array}$ & $\begin{array}{l}\text { Mar. } \\
\text { Proj. }\end{array}$ & $\begin{array}{l}\text { Jun. } \\
\text { Proj. }\end{array}$ & $\begin{array}{l}\text { Sep. } \\
\text { Proj. }\end{array}$ & $\begin{array}{l}\text { Dec. } \\
\text { Proj. }\end{array}$ \\
\hline & \multicolumn{10}{|c|}{ (In millions of soms) } \\
\hline Net foreign assets & 21,072 & 31,544 & 28,826 & 31,400 & 36,405 & 36,023 & 34,014 & 34,738 & 36,742 & 38,710 \\
\hline Net international reserves & 23,197 & 34,430 & 31,287 & 33,783 & 38,538 & 38,323 & 36,315 & 37,039 & 39,043 & 41,011 \\
\hline Long-term foreign liabilities & $-2,184$ & $-2,181$ & $-2,047$ & $-2,044$ & $-2,058$ & $-2,050$ & $-2,050$ & $-2,050$ & $-2,050$ & $-2,050$ \\
\hline Other foreign assets & 111 & -654 & -368 & -291 & -24 & -200 & -200 & -200 & -200 & -200 \\
\hline Balances with CIS countries & -51 & -51 & -46 & -48 & -51 & -51 & -51 & -51 & -51 & -51 \\
\hline Net domestic assets & 1,727 & 39 & 292 & 1,140 & $-1,459$ & 1,121 & 1,272 & 2,000 & 2,840 & 4,005 \\
\hline Net claims on general government & 1,802 & 767 & 531 & 1,001 & -136 & 2,010 & 1,913 & 2,313 & 2,113 & 2,759 \\
\hline Of which: total government deposits & $-5,242$ & $-4,994$ & $-4,616$ & $-4,111$ & $-5,271$ & $-3,144$ & $-3,244$ & $-2,844$ & $-3,044$ & $-2,395$ \\
\hline Repos & -504 & -193 & -182 & -231 & -10 & -10 & -10 & -10 & 289 & 812 \\
\hline Claims on commercial banks & 313 & 305 & 302 & 301 & 300 & 297 & 294 & 292 & 290 & 286 \\
\hline Other items (net) & 117 & -840 & -360 & 69 & $-1,612$ & $-1,177$ & -925 & -596 & 148 & 148 \\
\hline Reserve money & 22,799 & 31,583 & 29,117 & 32,540 & 34,947 & 37,144 & 35,286 & 36,738 & 39,582 & 42,715 \\
\hline Currency in circulation & 19,910 & 27,562 & 25,605 & 28,418 & 30,613 & 32,761 & 31,123 & 32,440 & 34,951 & 38,358 \\
\hline Commercial banks' reserves and other balances & 2,889 & 4,021 & 3,512 & 4,122 & 4,333 & 4,383 & 4,164 & 4,298 & 4,631 & 4,357 \\
\hline \multirow[t]{2}{*}{ Of which: required reserves } & 1,522 & 2,195 & 2,226 & 2,515 & 2,563 & 2,752 & 2,701 & 3,054 & 3,180 & 3,438 \\
\hline & \multicolumn{10}{|c|}{ (Annual percent change) } \\
\hline Net foreign assets & 33.7 & 49.7 & 35.7 & 23.8 & 23.5 & 14.2 & 18.0 & 10.6 & 0.9 & 7.5 \\
\hline $\begin{array}{l}\text { Net domestic assets } \\
\text { Of which: }\end{array}$ & -687.6 & -107.9 & -68.9 & 781.9 & -29.8 & $2,789.1$ & 336.1 & 75.4 & -294.7 & 257.3 \\
\hline Net claims on general government & 151.9 & -57.4 & -37.3 & 809.9 & -91.6 & 162.1 & 260.1 & 131.2 & -1652.2 & 37.3 \\
\hline Claims on commercial banks & -172.2 & -158.3 & 122.0 & 133.4 & -161.1 & 157.5 & 136.6 & 300.1 & 100.4 & 282.1 \\
\hline Reserve money & 47.5 & 38.5 & 31.3 & 27.6 & 27.5 & 17.6 & 21.2 & 12.9 & 13.3 & 15.0 \\
\hline Currency in circulation & 48.4 & 38.4 & 31.8 & 26.4 & 25.8 & 18.9 & 21.5 & 14.2 & 14.2 & 17.1 \\
\hline \multirow[t]{2}{*}{ Commercial banks' reserves and other balances } & 41.1 & 41.6 & 24.4 & 37.0 & 40.9 & 9.0 & 18.6 & 4.3 & 6.9 & -0.6 \\
\hline & \multicolumn{10}{|c|}{ (Contribution to reserve money growth, in percent) 2/ } \\
\hline Net foreign assets & 34.4 & 45.9 & -8.6 & -0.5 & 15.4 & 14.2 & -5.4 & -3.5 & 1.9 & 7.2 \\
\hline Net domestic assets & 13.1 & 2.2 & 0.8 & 3.5 & -4.7 & 3.4 & 0.4 & 2.4 & 4.6 & 7.8 \\
\hline Of which: net claims on general government & 7.0 & -4.5 & -0.7 & 0.7 & -2.9 & 3.9 & -0.3 & 0.8 & 0.3 & 2.0 \\
\hline Reserve money & 47.5 & 38.5 & -7.8 & 3.0 & 10.7 & 17.6 & -5.0 & -1.1 & 6.6 & 15.0 \\
\hline Of which: currency in circulation & 42.0 & 33.6 & -6.2 & 2.7 & 9.7 & 16.5 & -4.4 & -0.9 & 5.9 & 15.1 \\
\hline \multicolumn{11}{|l|}{ Memorandum items: } \\
\hline Net international reserves (in millions of dollars) & 607 & 901 & 819 & 884 & 1,009 & 1,003 & 950 & 969 & 1,022 & 1,073 \\
\hline Net domestic assets (in millions of soms) $3 /$ & $-2,541$ & $-2,036$ & $-1,781$ & -948 & $-3,561$ & -994 & -839 & -110 & 732 & 1,902 \\
\hline Reserve assets (in millions of dollars) & 773 & 1,043 & 952 & 1,031 & 1,145 & 1,162 & 1,100 & 1,141 & 1,185 & 1,260 \\
\hline
\end{tabular}

Source: Kyrgyz authorities; and Fund staff estimates and projections.

1/ Based on new program exchange rates (including 38.21 Som/US dollar) to be specified in the TMU.

$2 /$ Contribution is defined as change of asset stock relative to previous end-year reserve money stock (in percent).

3/ Excludes medium- and long-term central bank liabilities (i.e.the loan by the Eximbank of Turkey and the EBRD/IDA enterprise loan channeled through the NBKR). 
Table 4. Kyrgyz Republic: Monetary Survey, 2006-09 1/ 2/

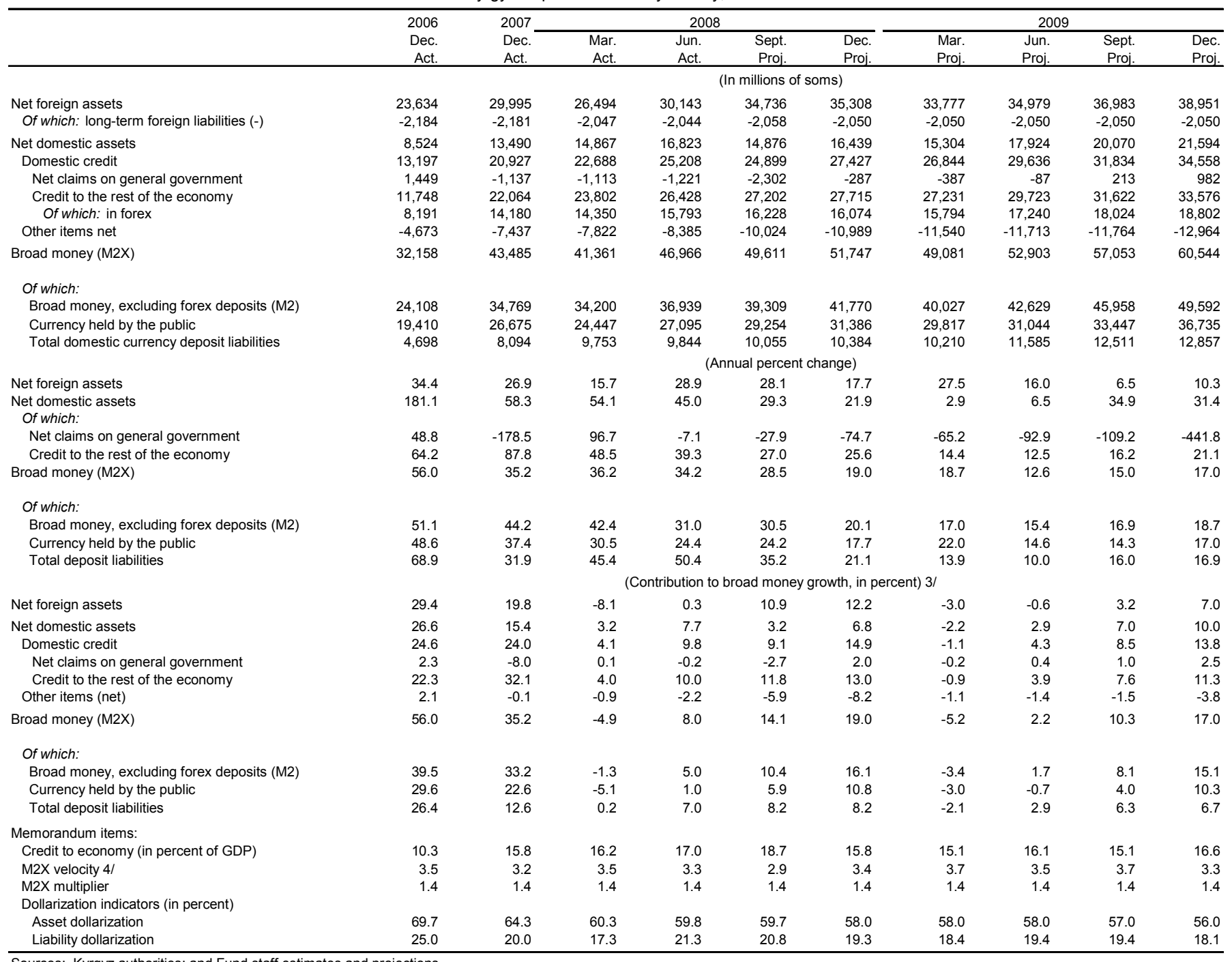

Sources: Kyrgyz authorities; and Fund staff estimates and projections.

1/ The monetary survey has been augmented since March 2007 to include the balances of Ayil Bank (formerly KAFC, a NBFI), which introduces a break into the series. 2/ Based on new program exchange rates (including 38.21 Som/US dollar) to be specified in the TMU.

$3 /$ Contribution is defined as change of asset stock relative to previous end-year broad money stock (in percent).

4/ 12-month GDP over end-period broad money. 
Table 5. Kyrgyz Republic: General Government Finances, 2006-09

\begin{tabular}{|c|c|c|c|c|c|c|c|c|}
\hline & \multirow[t]{2}{*}{2006} & \multirow[t]{2}{*}{2007} & \multicolumn{5}{|c|}{2008} & $20091 /$ \\
\hline & & & Q1 & Q2 & $\begin{array}{l}\text { Q3 } \\
\text { Est. }\end{array}$ & $\begin{array}{r}\text { Q4 } \\
\text { Proj. }\end{array}$ & $\begin{array}{l}\text { Year } \\
\text { Proj. }\end{array}$ & Proj. \\
\hline & \multicolumn{8}{|c|}{ (In millions of soms, unless indicated otherwise) } \\
\hline Total revenue and grants & 30,062 & 43,039 & 11,001 & 14,109 & 14,507 & 16,181 & 55,798 & 57,697 \\
\hline Total revenue & 29,174 & 39,821 & 10,840 & 13,019 & 13,959 & 13,319 & 51,137 & 52,374 \\
\hline Current revenue & 28,955 & 39,200 & 10,619 & 12,580 & 13,630 & 13,149 & 49,978 & 51,924 \\
\hline Tax revenue & 24,317 & 32,005 & 9,098 & 10,096 & 11,697 & 11,100 & 41,990 & 44,887 \\
\hline Income tax & 3,506 & 4,338 & 1,471 & 1,417 & 2,239 & 1,585 & 6,712 & 7,375 \\
\hline VAT 1/ & 9,151 & 12,702 & 3,455 & 4,121 & 4,350 & 4,588 & 16,514 & 13,916 \\
\hline Excises & 1,205 & 1,448 & 362 & 340 & 438 & 474 & 1,614 & 1,636 \\
\hline Customs & 2,803 & 3,789 & 936 & 1,140 & 1,131 & 1,525 & 4,732 & 6,285 \\
\hline Land tax & 412 & 587 & 147 & 146 & 170 & 188 & 651 & 765 \\
\hline Road tax and Emergency Fund 1/ & 1,534 & 1,913 & 613 & 623 & 850 & 771 & 2,857 & 0 \\
\hline Retail sales tax $1 /$ & 772 & 1,121 & 314 & 369 & 413 & 440 & 1,536 & 0 \\
\hline New turnover tax $1 /$ & $\ldots$ & $\ldots$ & $\ldots$ & $\ldots$ & $\ldots$ & $\ldots$ & $\ldots$ & 5,120 \\
\hline Social Fund (excl. gov. contribution) & 4,336 & 5,460 & 1,586 & 1,683 & 1,744 & 1,143 & 6,156 & 6,793 \\
\hline Other & 599 & 646 & 216 & 256 & 362 & 385 & 1,219 & 2,998 \\
\hline Nontax revenue & 4,637 & 7,195 & 1,521 & 2,484 & 1,934 & 2,049 & 7,988 & 7,037 \\
\hline Capital revenue & 220 & 466 & 222 & 440 & 328 & 170 & 1,160 & 450 \\
\hline Grants & 888 & 3,218 & 161 & 1,090 & 548 & 2,862 & 4,661 & 5,323 \\
\hline Program grants & 266 & 1,790 & 0 & 544 & 63 & 1,137 & 1,744 & 1,609 \\
\hline PIP grants & 622 & 1,429 & 161 & 546 & 485 & 1,725 & 2,917 & 3,714 \\
\hline Total expenditure (including net lending) & 32,922 & 44,020 & 9,335 & 14,511 & 13,905 & 20,379 & 58,130 & 61,221 \\
\hline Total expenditure (excluding net lending) & 33,149 & 43,915 & 8,854 & 14,731 & 13,652 & 20,256 & 57,494 & 61,523 \\
\hline Current expenditure & 28,232 & 37,170 & 7,935 & 12,679 & 11,039 & 14,456 & 46,109 & 47,519 \\
\hline Wages & 7,451 & 9,928 & 1,916 & 3,405 & 3,051 & 4,364 & 12,736 & 13,490 \\
\hline Transfers and subsidies & 4,201 & 5,047 & 1,331 & 1,893 & 1,419 & 1,242 & 5,886 & 5,535 \\
\hline Social Fund expenditures & 6,421 & 7,495 & 1,970 & 2,186 & 2,440 & 2,958 & 9,554 & 12,036 \\
\hline Interest & 1,002 & 916 & 145 & 719 & 164 & 371 & 1,399 & 1,709 \\
\hline Purchases of other goods and services & 9,157 & 13,783 & 2,572 & 4,476 & 3,965 & 5,521 & 16,534 & 14,749 \\
\hline Capital expenditure & 4,918 & 6,745 & 919 & 2,052 & 2,613 & 5,801 & 11,385 & 14,004 \\
\hline Domestically financed capital expenditure & 1,287 & 3,112 & 462 & 1,269 & 1,715 & 2,937 & 6,383 & 6,877 \\
\hline Foreign financed PIP loans & 3,035 & 2,205 & 296 & 238 & 412 & 1,139 & 2,085 & 3,414 \\
\hline Foreign financed PIP grants & 596 & 1,429 & 161 & 546 & 485 & 1,725 & 2,917 & 3,714 \\
\hline Net lending & -227 & 105 & 481 & -220 & 253 & 122 & 636 & -302 \\
\hline Discrepancy & 447 & 538 & $-1,829$ & 141 & -16 & 1,702 & 0 & 0 \\
\hline Primary balance excluding grant & $-2,298$ & $-2,746$ & -178 & -632 & 201 & $-4,987$ & $-5,594$ & $-7,139$ \\
\hline Primary balance & $-1,411$ & 472 & -17 & 458 & 750 & $-2,125$ & -933 & $-1,816$ \\
\hline Overall balance & $-2,413$ & -444 & -162 & -261 & 586 & $-2,496$ & $-2,332$ & $-3,525$ \\
\hline Total financing & 2,413 & 444 & 162 & 261 & -586 & 2,496 & 2,332 & 3,525 \\
\hline External financing & 2,837 & 1,911 & 120 & 309 & 333 & 960 & 1,722 & 2,044 \\
\hline Domestic financing & -445 & $-1,671$ & 29 & -65 & -936 & 1,824 & 850 & 1,269 \\
\hline NBKR & 317 & $-1,234$ & -215 & 493 & $-1,036$ & 2,001 & 1,243 & 749 \\
\hline Commercial banks & -700 & -437 & 243 & -568 & 52 & -121 & -393 & 520 \\
\hline Nonbank & -63 & 1 & 1 & 9 & 48 & -58 & 0 & 0 \\
\hline Privatization & 21 & 203 & 14 & 18 & 18 & -289 & -240 & 212 \\
\hline
\end{tabular}

Sources: Kyrgyz authorities; and Fund staff estimates and projections.

$1 /$ In 2009, the road, emergency and retail taxes will be abolished, the VAT tax rate will decline from 20 percent to 12 percent, and a new turnover tax will be introduced. 
Table 6. Kyrgyz Republic: General Government Finances, 2006-09

\begin{tabular}{|c|c|c|c|c|c|c|c|c|}
\hline & \multirow[t]{2}{*}{2006} & \multirow[t]{2}{*}{2007} & \multicolumn{5}{|c|}{2008} & \multirow{2}{*}{$\begin{array}{l}2009 \\
\text { Year } \\
\text { Proj. }\end{array}$} \\
\hline & & & Q1 & Q2 & $\begin{array}{c}\text { Q3 } \\
\text { Est. }\end{array}$ & $\begin{array}{r}\text { Q4 } \\
\text { Proj }\end{array}$ & $\begin{array}{l}\text { Year } \\
\text { Proj. }\end{array}$ & \\
\hline & \multicolumn{8}{|c|}{ (percent of GDP) } \\
\hline Total revenue and grants & 26.4 & 30.8 & 39.1 & 37.9 & 37.0 & 22.7 & 31.7 & 28.5 \\
\hline Total revenue & 25.6 & 28.5 & 38.5 & 35.0 & 35.6 & 18.7 & 29.1 & 25.9 \\
\hline Current revenue & 25.4 & 28.1 & 37.7 & 33.8 & 34.8 & 18.5 & 28.4 & 25.7 \\
\hline Tax revenue & 21.4 & 22.9 & 32.3 & 27.1 & 29.9 & 15.6 & 23.9 & 22.2 \\
\hline Income tax & 3.1 & 3.1 & 5.2 & 3.8 & 5.7 & 2.2 & 3.8 & 3.6 \\
\hline VAT 1/ & 8.0 & 9.1 & 12.3 & 11.1 & 11.1 & 6.4 & 9.4 & 6.9 \\
\hline Excises & 1.1 & 1.0 & 1.3 & 0.9 & 1.1 & 0.7 & 0.9 & 0.8 \\
\hline Customs & 2.5 & 2.7 & 3.3 & 3.1 & 2.9 & 2.1 & 2.7 & 3.1 \\
\hline Land tax & 0.4 & 0.4 & 0.5 & 0.4 & 0.4 & 0.3 & 0.4 & 0.4 \\
\hline Road tax and Emergency Fund 1/ & 1.3 & 1.4 & 2.2 & 1.7 & 2.2 & 1.1 & 1.6 & 0.0 \\
\hline Retail sales tax $1 /$ & 0.7 & 0.8 & 1.1 & 1.0 & 1.1 & 0.6 & 0.9 & 0.0 \\
\hline New turnover tax $1 /$ & $\ldots$ & $\ldots$ & 0.0 & 0.0 & 0.0 & 0.0 & $\ldots$ & 2.5 \\
\hline Social Fund (excl. gov. contribution) & 3.8 & 3.9 & 5.6 & 4.5 & 4.5 & 1.6 & 3.5 & 3.4 \\
\hline Other & 0.5 & 0.5 & 0.8 & 0.7 & 0.9 & 0.5 & 0.7 & 1.5 \\
\hline Nontax revenue & 4.1 & 5.1 & 5.4 & 6.7 & 4.9 & 2.9 & 4.5 & 3.5 \\
\hline Capital revenue & 0.2 & 0.3 & 0.8 & 1.2 & 0.8 & 0.2 & 0.7 & 0.2 \\
\hline Grants & 0.8 & 2.3 & 0.6 & 2.9 & 1.4 & 4.0 & 2.7 & 2.6 \\
\hline Program grants & 0.2 & 1.3 & 0.0 & 1.5 & 0.2 & 1.6 & 1.0 & 0.8 \\
\hline PIP grants & 0.5 & 1.0 & 0.6 & 1.5 & 1.2 & 2.4 & 1.7 & 1.8 \\
\hline Total expenditure (including net lending) & 28.9 & 31.5 & 33.2 & 39.0 & 35.5 & 28.6 & 33.1 & 30.2 \\
\hline Total expenditure (excluding net lending) & 29.1 & 31.4 & 31.5 & 39.6 & 34.8 & 28.4 & 32.7 & 30.4 \\
\hline Current expenditure & 24.8 & 26.6 & 28.2 & 34.1 & 28.2 & 20.3 & 26.2 & 23.5 \\
\hline Wages & 6.5 & 7.1 & 6.8 & 9.2 & 7.8 & 6.1 & 7.2 & 6.7 \\
\hline Transfers and subsidies & 3.7 & 3.6 & 4.7 & 5.1 & 3.6 & 1.7 & 3.3 & 2.7 \\
\hline Social Fund expenditures & 5.6 & 5.4 & 7.0 & 5.9 & 6.2 & 4.2 & 5.4 & 5.9 \\
\hline Interest & 0.9 & 0.7 & 0.5 & 1.9 & 0.4 & 0.5 & 0.8 & 0.8 \\
\hline Purchases of other goods and services & 8.0 & 9.9 & 9.1 & 12.0 & 10.1 & 7.7 & 9.4 & 7.3 \\
\hline Capital expenditure & 4.3 & 4.8 & 3.3 & 5.5 & 6.7 & 8.1 & 6.5 & 6.9 \\
\hline Domestically financed capital expenditure & 1.1 & 2.2 & 1.6 & 3.4 & 4.4 & 4.1 & 3.6 & 3.4 \\
\hline Foreign financed PIP loans & 2.7 & 1.6 & 1.1 & 0.6 & 1.1 & 1.6 & 1.2 & 1.7 \\
\hline Foreign financed PIP grants & 0.5 & 1.0 & 0.6 & 1.5 & 1.2 & 2.4 & 1.7 & 1.8 \\
\hline Net lending & -0.2 & 0.1 & 1.7 & -0.6 & 0.6 & 0.2 & 0.4 & -0.1 \\
\hline Discrepancy & 0.4 & 0.4 & -6.5 & 0.4 & 0.0 & 2.4 & 0.0 & 0.0 \\
\hline Primary balance excluding grant & -2.0 & -2.0 & -0.6 & -1.7 & 0.5 & -7.0 & -3.2 & -3.5 \\
\hline Primary balance & -1.2 & 0.3 & -0.1 & 1.2 & 1.9 & -3.0 & -0.5 & -0.9 \\
\hline Overall balance & -2.1 & -0.3 & -0.6 & -0.7 & 1.5 & -3.5 & -1.3 & -1.7 \\
\hline Total financing & 2.1 & 0.3 & 0.6 & 0.7 & -1.5 & 3.5 & 1.3 & 1.7 \\
\hline External financing & 2.5 & 1.4 & 0.4 & 0.8 & 0.8 & 1.3 & 1.0 & 1.0 \\
\hline Domestic financing & -0.4 & -1.2 & 0.1 & -0.2 & -2.4 & 2.6 & 0.5 & 0.6 \\
\hline NBKR & 0.3 & -0.9 & -0.8 & 1.3 & -2.6 & 2.8 & 0.7 & 0.4 \\
\hline Commercial banks & -0.6 & -0.3 & 0.9 & -1.5 & 0.1 & -0.2 & -0.2 & 0.3 \\
\hline Nonbank & -0.1 & 0.0 & 0.0 & 0.0 & 0.1 & -0.1 & 0.0 & 0.0 \\
\hline Privatization & 0.0 & 0.1 & 0.0 & 0.0 & 0.0 & -0.4 & -0.1 & 0.1 \\
\hline
\end{tabular}

Sources: Kyrgyz authorities; and Fund staff estimates and projections.

$1 /$ In 2009, the road, emergency and retail taxes will be abolished, the VAT tax rate will decline from 20 percent to 12 percent, and a new turnover tax will be introduced. 
Table 7. Kyrgyz Republic: State Government Finances, 2006-09

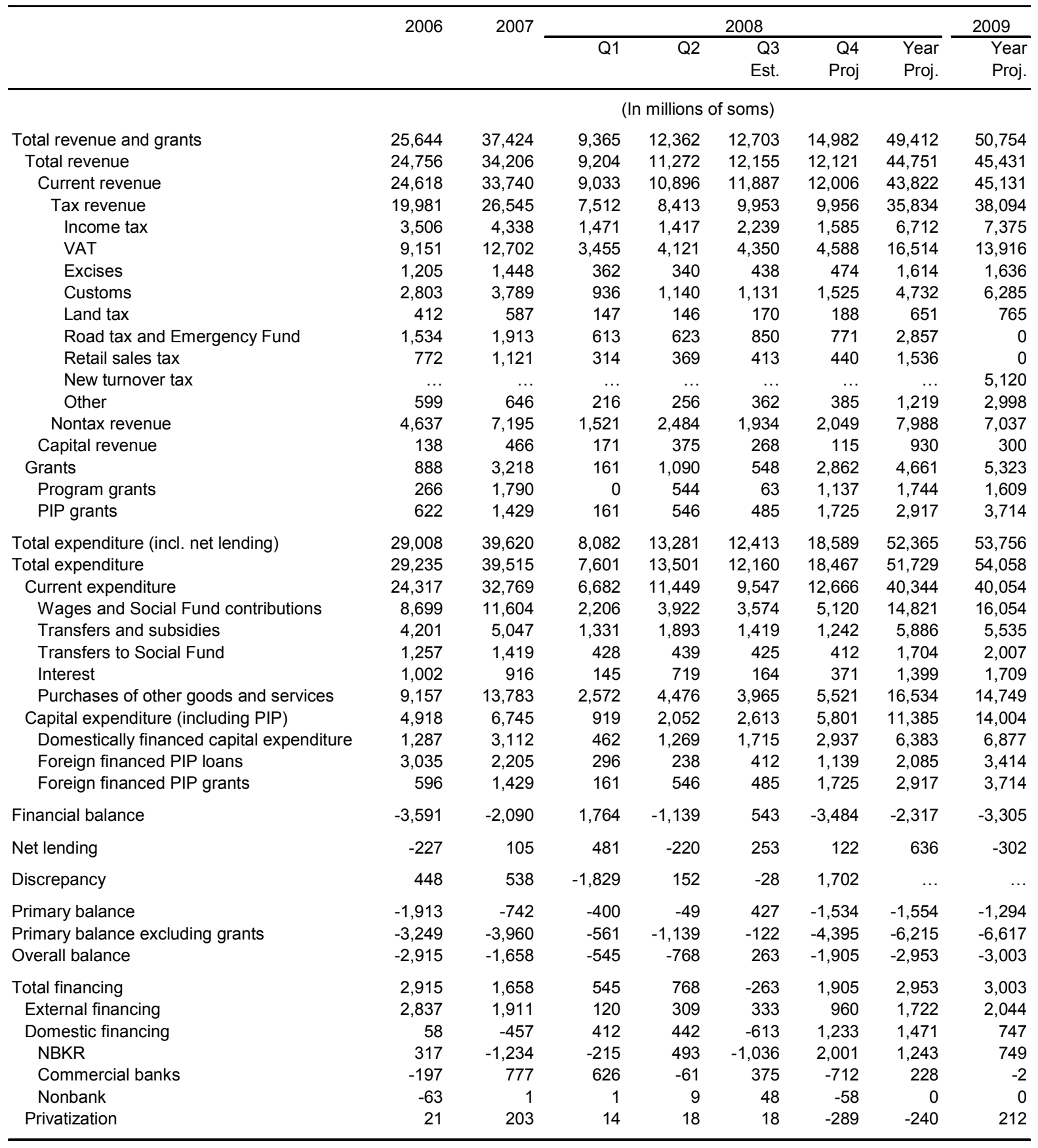

Sources: Kyrgyz authorities; and Fund staff estimates and projections. 
Table 8. Kyrgyz Republic: State Government Finances, 2006-09

\begin{tabular}{|c|c|c|c|c|c|c|c|c|}
\hline & \multirow[t]{2}{*}{2006} & \multirow[t]{2}{*}{2007} & \multicolumn{5}{|c|}{2008} & \multirow{2}{*}{$\begin{array}{l}\frac{2009}{\text { Year }} \\
\text { Proj. }\end{array}$} \\
\hline & & & Q1 & Q2 & $\begin{array}{r}\text { Q3 } \\
\text { Est. }\end{array}$ & $\begin{array}{r}\text { Q4 } \\
\text { Proj }\end{array}$ & $\begin{array}{l}\text { Year } \\
\text { Proj. }\end{array}$ & \\
\hline & \multicolumn{8}{|c|}{ (In percent of GDP) } \\
\hline Total revenue and grants & 22.5 & 26.8 & 33.3 & 33.2 & 32.4 & 21.0 & 28.1 & 25.1 \\
\hline Total revenue & 21.8 & 24.5 & 32.7 & 30.3 & 31.0 & 17.0 & 25.5 & 22.4 \\
\hline Current revenue & 21.6 & 24.1 & 32.1 & 29.3 & 30.3 & 16.9 & 24.9 & 22.3 \\
\hline Tax revenue & 17.6 & 19.0 & 26.7 & 22.6 & 25.4 & 14.0 & 20.4 & 18.8 \\
\hline Income tax & 3.1 & 3.1 & 5.2 & 3.8 & 5.7 & 2.2 & 3.8 & 3.6 \\
\hline VAT & 8.0 & 9.1 & 12.3 & 11.1 & 11.1 & 6.4 & 9.4 & 6.9 \\
\hline Excises & 1.1 & 1.0 & 1.3 & 0.9 & 1.1 & 0.7 & 0.9 & 0.8 \\
\hline Customs & 2.5 & 2.7 & 3.3 & 3.1 & 2.9 & 2.1 & 2.7 & 3.1 \\
\hline Land tax & 0.4 & 0.4 & 0.5 & 0.4 & 0.4 & 0.3 & 0.4 & 0.4 \\
\hline Road tax and Emergency Fund & 1.3 & 1.4 & 2.2 & 1.7 & 2.2 & 1.1 & 1.6 & 0.0 \\
\hline Retail sales tax & 0.7 & 0.8 & 1.1 & 1.0 & 1.1 & 0.6 & 0.9 & 0.0 \\
\hline New turnover tax & & $\ldots$ & $\ldots$ & & $\ldots$ & $\ldots$ & $\ldots$ & 2.5 \\
\hline Other & 0.5 & 0.5 & 0.8 & 0.7 & 0.9 & 0.5 & 0.7 & 1.5 \\
\hline Nontax revenue & 4.1 & 5.1 & 5.4 & 6.7 & 4.9 & 2.9 & 4.5 & 3.5 \\
\hline Capital revenue & 0.1 & 0.3 & 0.6 & 1.0 & 0.7 & 0.2 & 0.5 & 0.1 \\
\hline Grants & 0.8 & 2.3 & 0.6 & 2.9 & 1.4 & 4.0 & 2.7 & 2.6 \\
\hline Program grants & 0.2 & 1.3 & 0.0 & 1.5 & 0.2 & 1.6 & 1.0 & 0.8 \\
\hline PIP grants & 0.5 & 1.0 & 0.6 & 1.5 & 1.2 & 2.4 & 1.7 & 1.8 \\
\hline Total expenditure (incl. net lending) & 25.5 & 28.4 & 28.7 & 35.7 & 31.7 & 26.1 & 29.8 & 26.6 \\
\hline Total expenditure & 25.7 & 28.3 & 27.0 & 36.3 & 31.0 & 25.9 & 29.4 & 26.7 \\
\hline Current expenditure & 21.4 & 23.4 & 23.7 & 30.8 & 24.4 & 17.8 & 23.0 & 19.8 \\
\hline Wages and Social Fund contributions & 7.6 & 8.3 & 7.8 & 10.5 & 9.1 & 7.2 & 8.4 & 7.9 \\
\hline Transfers and subsidies & 3.7 & 3.6 & 4.7 & 5.1 & 3.6 & 1.7 & 3.3 & 2.7 \\
\hline Transfers to Social Fund & 1.1 & 1.0 & 1.5 & 1.2 & 1.1 & 0.6 & 1.0 & 1.0 \\
\hline Interest & 0.9 & 0.7 & 0.5 & 1.9 & 0.4 & 0.5 & 0.8 & 0.8 \\
\hline Purchases of other goods and services & 8.0 & 9.9 & 9.1 & 12.0 & 10.1 & 7.7 & 9.4 & 7.3 \\
\hline Capital expenditure (including PIP) & 4.3 & 4.8 & 3.3 & 5.5 & 6.7 & 8.1 & 6.5 & 6.9 \\
\hline Domestically financed capital expenditure & 1.1 & 2.2 & 1.6 & 3.4 & 4.4 & 4.1 & 3.6 & 3.4 \\
\hline Foreign financed PIP loans & 2.7 & 1.6 & 1.1 & 0.6 & 1.1 & 1.6 & 1.2 & 1.7 \\
\hline Foreign financed PIP grants & 0.5 & 1.0 & 0.6 & 1.5 & 1.2 & 2.4 & 1.7 & 1.8 \\
\hline Financial balance & -3.2 & -1.5 & 6.3 & -3.1 & 1.4 & -4.9 & -1.3 & -1.6 \\
\hline Net lending & -0.2 & 0.1 & 1.7 & -0.6 & 0.6 & 0.2 & 0.4 & -0.1 \\
\hline Discrepancy & 0.4 & 0.4 & -6.5 & 0.4 & -0.1 & 2.4 & $\ldots$ & $\ldots$ \\
\hline Primary balance & -1.7 & -0.5 & -1.4 & -0.1 & 1.1 & -2.2 & -0.9 & -0.6 \\
\hline Primary balance excluding grants & -2.9 & -2.8 & -2.0 & -3.1 & -0.3 & -6.2 & -3.5 & -3.3 \\
\hline Overall balance & -2.6 & -1.2 & -1.9 & -2.1 & 0.7 & -2.7 & -1.7 & -1.5 \\
\hline Total financing & 2.6 & 1.2 & 1.9 & 2.1 & -0.7 & 2.7 & 1.7 & 1.5 \\
\hline External financing & 2.5 & 1.4 & 0.4 & 0.8 & 0.8 & 1.3 & 1.0 & 1.0 \\
\hline Domestic financing & 0.1 & -0.3 & 1.5 & 1.2 & -1.6 & 1.7 & 0.8 & 0.4 \\
\hline NBKR & 0.3 & -0.9 & -0.8 & 1.3 & -2.6 & 2.8 & 0.7 & 0.4 \\
\hline Commercial banks & -0.2 & 0.6 & 2.2 & -0.2 & 1.0 & -1.0 & 0.1 & 0.0 \\
\hline Nonbank & -0.1 & 0.0 & 0.0 & 0.0 & 0.1 & -0.1 & 0.0 & 0.0 \\
\hline Privatization & 0.0 & 0.1 & 0.0 & 0.0 & 0.0 & -0.4 & -0.1 & 0.1 \\
\hline
\end{tabular}

Sources: Kyrgyz authorities; and Fund staff estimates and projections. 
Table 9. Kyrgyz Republic: Social Fund Operations, 2006-09

\begin{tabular}{|c|c|c|c|c|c|c|c|c|}
\hline & \multirow[t]{2}{*}{2006} & \multirow[t]{2}{*}{2007} & \multicolumn{5}{|c|}{2008} & \multirow{2}{*}{$\begin{array}{l}2009 \\
\text { Year } \\
\text { Proj. }\end{array}$} \\
\hline & & & Q1 & Q2 & $\begin{array}{l}\text { Q3 } \\
\text { Prelim. }\end{array}$ & $\begin{array}{c}\text { Q4 } \\
\text { Proj. }\end{array}$ & $\begin{array}{l}\text { Year } \\
\text { Proj. }\end{array}$ & \\
\hline & \multicolumn{8}{|c|}{ (millions of soms) } \\
\hline Total revenue & 5,667 & 7,290 & 1,926 & 2,264 & 2,326 & 1,955 & 8,471 & 9,507 \\
\hline Total contribution & 5,585 & 7,136 & 1,875 & 2,200 & 2,266 & 1,900 & 8,241 & 9,357 \\
\hline Other revenue & 82 & 155 & 51 & 64 & 60 & 55 & 230 & 150 \\
\hline Total expenditure & 6,421 & 7,495 & 1,970 & 2,186 & 2,440 & 2,958 & 9,554 & 12,036 \\
\hline Pension Fund & 6,100 & 7,088 & 1,883 & 2,081 & 2,284 & 2,796 & 9,045 & 11,412 \\
\hline Medical Insurance Fund (incl. old Funds) & 321 & 407 & 87 & 105 & 156 & 162 & 509 & 597 \\
\hline Insurance payments from funded pillar & $\ldots$ & $\ldots$ & $\ldots$ & $\ldots$ & $\ldots$ & $\ldots$ & $\ldots$ & 27 \\
\hline Financial balance & -754 & -205 & -45 & 79 & -113 & $-1,004$ & $-1,083$ & $-2,529$ \\
\hline Budgetary transfer & 1,257 & 1,419 & 428 & 428 & 436 & 412 & 1,704 & 2,007 \\
\hline Overall balance & 503 & 1,214 & 383 & 507 & 323 & -591 & 621 & -522 \\
\hline Financing & -503 & $-1,214$ & -383 & -507 & -323 & 591 & -621 & 522 \\
\hline Commercial bank deposits & -503 & $-1,214$ & -383 & -507 & -323 & 591 & -621 & 823 \\
\hline \multirow[t]{2}{*}{ Funded Pillar } & 0 & 0 & 0 & 0 & 0 & 0 & 0 & -301 \\
\hline & \multicolumn{8}{|c|}{ (percent of GDP) } \\
\hline Total revenue & 5.0 & 5.2 & 6.8 & 6.1 & 5.9 & 2.7 & 4.8 & 4.7 \\
\hline Total contribution & 4.9 & 5.1 & 6.7 & 5.9 & 5.8 & 2.7 & 4.7 & 4.6 \\
\hline Other revenue & 0.1 & 0.1 & 0.2 & 0.2 & 0.2 & 0.1 & 0.1 & 0.1 \\
\hline Total expenditure & 5.6 & 5.4 & 7.0 & 5.9 & 6.2 & 4.2 & 5.4 & 5.9 \\
\hline Pension Fund & 5.4 & 5.1 & 6.7 & 5.6 & 5.8 & 3.9 & 5.1 & 5.6 \\
\hline Medical Insurance Fund (incl. old Funds) & 0.3 & 0.3 & 0.3 & 0.3 & 0.4 & 0.2 & 0.3 & 0.3 \\
\hline Insurance payments from funded pillar & $\ldots$ & $\ldots$ & $\ldots$ & $\ldots$ & $\ldots$ & $\ldots$ & $\ldots$ & 0.0 \\
\hline Financial balance & -0.7 & -0.1 & -0.2 & 0.2 & -0.3 & -1.4 & -0.6 & -1.2 \\
\hline Budgetary transfer & 1.1 & 1.0 & 1.5 & 1.2 & 1.1 & 0.6 & 1.0 & 1.0 \\
\hline Overall balance & 0.4 & 0.9 & 1.4 & 1.4 & 0.8 & -0.8 & 0.4 & -0.3 \\
\hline Financing & -0.4 & -0.9 & -1.4 & -1.4 & -0.8 & 0.8 & -0.4 & 0.3 \\
\hline Commercial bank deposits & -0.4 & -0.9 & -1.4 & -1.4 & -0.8 & 0.8 & -0.4 & 0.4 \\
\hline Funded Pillar & 0.0 & 0.0 & 0.0 & 0.0 & 0.0 & 0.0 & 0.0 & -0.1 \\
\hline
\end{tabular}

Sources: Kyrgyz authorities; and Fund staff estimates and projections. 
Table 10. Kyrgyz Republic: Quantitative Program Targets

(In millions of soms, unless otherwise indicated; eop)

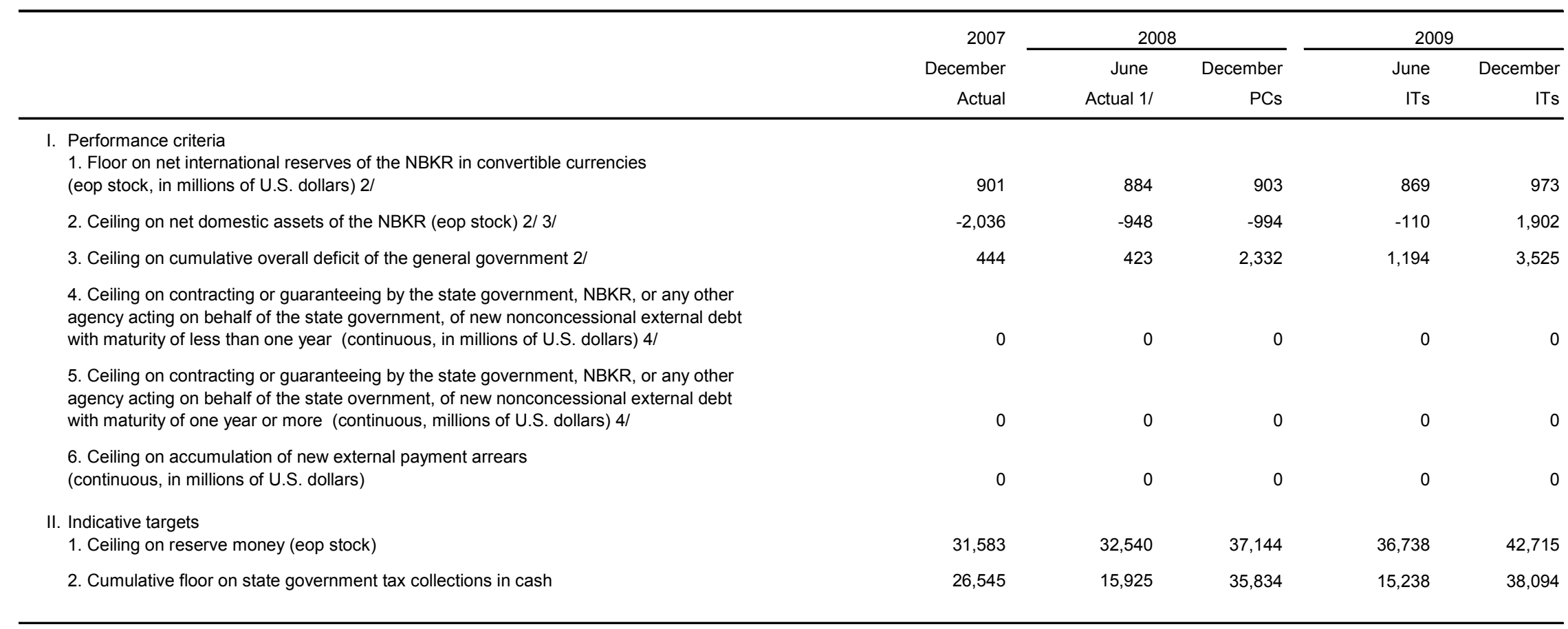

Sources: Kyrgyz authorities; and Fund staff estimates and projections.

1/ Preliminary data for fiscal outcome.

2/ These targets are subject to adjustment in case of changes in flows of external budget financing by bilateral and multilateral donors as defined in the TMU.

3/ The target excludes medium- and long-term central bank liabilities (i.e., the loan by the Eximbank of Turkey and the EBRD/IDA enterprise loan channeled through the NBKR).

4/ External debt contracted or guaranteed other than with a grant element equivalent to 35 percent or more, calculated using a discount rate based on the OECD commercial

interest reference rates. Excludes borrowing from the IMF. 
Table 11. Kyrgyz Republic: Indicators of Capacity to Repay the Fund, 2007-15 1/ Access to a new ESF arrangement at 75 percent of quota

\begin{tabular}{|c|c|c|c|c|c|c|c|c|c|}
\hline & 2007 & 2008 & 2009 & 2010 & 2011 & 2012 & 2013 & 2014 & 2015 \\
\hline & Actual & & & & Projec & & & & \\
\hline \multicolumn{10}{|l|}{$\begin{array}{l}\text { Fund obligations based on existing credit } \\
\text { (in millions of SDRs) }\end{array}$} \\
\hline Principal & 16.1 & 14.8 & 14.7 & 16.2 & 15.3 & 13.5 & 11.4 & 8.3 & 4.4 \\
\hline Charges and interest & 0.5 & 0.5 & 0.4 & 0.3 & 0.3 & 0.2 & 0.1 & 0.1 & 0.0 \\
\hline \multicolumn{10}{|l|}{$\begin{array}{l}\text { Fund obligations based on existing and prospective credit } \\
\text { (in millions of SDRs) }\end{array}$} \\
\hline Principal & 16.1 & 14.8 & 14.7 & 16.2 & 15.3 & 13.5 & 11.4 & 13.3 & 16.0 \\
\hline Charges and interest & 0.5 & 0.5 & 0.6 & 0.7 & 0.6 & 0.5 & 0.5 & 0.4 & 0.3 \\
\hline \multicolumn{10}{|l|}{ Total obligations based on existing and prospective credit } \\
\hline In millions of SDRs & 16.6 & 15.3 & 15.3 & 16.9 & 15.9 & 14.0 & 11.8 & 13.7 & 16.4 \\
\hline In millions of US\$ & 25.5 & 24.7 & 24.9 & 27.5 & 26.0 & 22.9 & 19.4 & 22.5 & 26.9 \\
\hline In percent of Gross International Reserves & 2.1 & 2.1 & 2.0 & 1.9 & 1.6 & 1.2 & 0.9 & 1.0 & 1.1 \\
\hline In percent of exports of goods and services & 1.1 & 0.8 & 0.8 & 0.8 & 0.7 & 0.5 & 0.4 & 0.4 & 0.5 \\
\hline In percent of debt service $2 /$ & 39.0 & 34.4 & 28.6 & 29.5 & 27.0 & 23.7 & 20.0 & 22.5 & 25.9 \\
\hline In percent of GDP & 0.7 & 0.5 & 0.5 & 0.5 & 0.4 & 0.3 & 0.3 & 0.3 & 0.3 \\
\hline In percent of quota & 18.7 & 17.2 & 17.2 & 19.0 & 17.9 & 15.8 & 13.3 & 15.5 & 18.4 \\
\hline \multicolumn{10}{|l|}{ Outstanding Fund credit $2 /$} \\
\hline In millions of SDRs & 94.8 & 106.8 & 125.4 & 125.8 & 110.5 & 97.0 & 85.7 & 72.3 & 56.3 \\
\hline In billions of US\$ & 0.1 & 0.2 & 0.2 & 0.2 & 0.2 & 0.2 & 0.1 & 0.1 & 0.1 \\
\hline In percent of Gross International Reserves & 12.2 & 14.9 & 16.2 & 14.1 & 10.9 & 8.5 & 6.7 & 5.4 & 3.9 \\
\hline In percent of exports of goods and services & 6.5 & 5.5 & 6.7 & 5.9 & 4.7 & 3.6 & 2.9 & 2.3 & 1.8 \\
\hline In percent of debt service $2 /$ & 222.2 & 240.8 & 234.0 & 219.4 & 187.9 & 164.3 & 145.4 & 118.5 & 89.2 \\
\hline In percent of GDP & 3.9 & 3.6 & 4.0 & 3.5 & 2.8 & 2.3 & 1.9 & 1.5 & 1.1 \\
\hline In percent of quota & 106.8 & 120.3 & 141.2 & 141.7 & 124.4 & 109.3 & 96.5 & 81.5 & 63.4 \\
\hline Net use of Fund credit (millions of SDRs) & $(13.6)$ & 12.0 & 18.6 & 0.4 & $(15.3)$ & $(13.5)$ & $(11.4)$ & (13.3) & (16.0) \\
\hline Disbursements & 2.5 & 26.8 & 33.3 & 16.7 & - & - & - & - & - \\
\hline Repayments and Repurchases & 16.1 & 14.8 & 14.7 & 16.2 & 15.3 & 13.5 & 11.4 & 13.3 & 16.0 \\
\hline \multicolumn{10}{|l|}{ Memorandum items: } \\
\hline Nominal GDP (in millions of US\$) & 3,748 & 4,805 & 5,124 & 5,838 & 6,370 & 6,992 & 7,550 & 8,066 & 8,412 \\
\hline Exports of goods and services (in millions of US $\$$ ) & 2,244 & 3,138 & 3,037 & 3,476 & 3,856 & 4,362 & 4,805 & 5,178 & 5,278 \\
\hline Gross International Reserves (in millions of US\$) & 1,194 & 1,162 & 1,260 & 1,456 & 1,651 & 1,858 & 2,094 & 2,214 & 2,364 \\
\hline Debt service (in millions of US $\$$ ) $2 /$ & 65.3 & 71.8 & 87.0 & 93.4 & 96.0 & 96.7 & 96.8 & 100.2 & 103.7 \\
\hline Quota (millions of SDRs) & 88.8 & 88.8 & 88.8 & 88.8 & 88.8 & 88.8 & 88.8 & 88.8 & 88.8 \\
\hline
\end{tabular}

Sources: IMF staff estimates and projections.

1/ Assumes a new ESF arrangement of 75 percent of quota (SDR 66.6 million).

2/ Total debt service includes IMF repurchases and repayments. 
Table 12. Kyrgyz Republic: Reviews and Disbursements Under the 18-month ESF Arrangement

\begin{tabular}{|c|c|c|}
\hline Date & Action & $\begin{array}{r}\text { Associated } \\
\text { Disbursement }\end{array}$ \\
\hline On or after December 10, 2008 & Approved 18-month ESF arrangement. & SDR 16.65 million \\
\hline On or after March 10, 2009 & Completed first review based on end-December 2008 performance criteria. & SDR 16.65 million \\
\hline On or after September 10, 2009 & Completed second review based on end-June 2009 performance criteria. & SDR 16.65 million \\
\hline $\begin{array}{l}\text { On or after March } 10,2010 \text {, but no } \\
\text { later than June } 10,2010\end{array}$ & Completed third review based on end-December 2009 performance criteria. & SDR 16.65 million \\
\hline Total & & SDR 66.60 million \\
\hline
\end{tabular}

Source: International Monetary Fund. 


\section{ATTACHMENT I. LETTER OF INTENT}

November 25, 2008

Mr. Dominique Strauss-Kahn

Managing Director

International Monetary Fund

$70019^{\text {th }}$ Street, N.W.

Washington, D.C. 20431

\section{Dear Mr. Strauss-Kahn:}

In the past few years, the Kyrgyz Republic's economic performance strengthened considerably, thanks to improved macroeconomic management and a favorable external environment that lasted until mid-2007. These improvements also reflected the assistance we received from the international community, including from the International Monetary Fund. Growth accelerated, poverty declined, inflation remained low, and our fiscal and external sustainability improved dramatically. Our last arrangement with the IMF under the Poverty Reduction and Growth Facility (PRGF) was successfully completed in May of this year.

Already at the time of the last PRGF review it was evident that the external environment was deteriorating rapidly, with the sharp rise in international food and fuel prices and the onset of a slowdown in the global and regional economy. Because of this, we requested additional resources from the IMF with the final review. Since then, these shocks have intensified, while other shocks have emerged. International food and fuel prices continued to rise in the first half of this year, causing inflation in the Kyrgyz Republic to surge. With the recent easing in international commodity prices, inflation has started to come down, but our import bill for food and energy products will still be significantly higher this year and next and many of our poorest households cannot cover their basic needs. In addition, water levels at our Toktogul hydropower station have dropped to well below what is needed for an uninterrupted energy supply this winter, forcing us to import large volumes of fossil fuels to avoid major power and heating shortages. The pace of economic growth in our neighbors continues to fall as the international financial crisis has continued to spread, adding to our balance of payments difficulties by affecting exports and remittances sent by our workers abroad. The effects of the international financial turmoil have spilled over on our banking system as well, and banks have significantly curtailed the extension of new loans.

Together, these shocks threaten to undermine our hard-won macroeconomic stability and reverse much of the gains that had been made in poverty reduction. To address the adverse consequences of these exogenous shocks, we have developed and adopted the attached Economic Program of the Kyrgyz Republic for 2008-2009, which describes our policies for the remainder of 2008 and 2009. Our program aims to manage the effects on the Kyrgyz 
economy of the slowdown in regional economic growth and potential spillovers from the international financial crisis. Our program focuses on reducing inflation, maintaining and accelerating growth, and ensuring targeted protection of the poor. Central to our efforts to reduce inflation has been a sharp tightening of monetary policy_-starting in the second half of last year and with a sharp increase in interest rates this summer - while fiscal policy is aiming to balance the need to help reducing inflation with the need to support growth and the poor. While our main focus is on macroeconomic policies, we have also accelerated the pace of structural reforms, to improve macroeconomic management and ensure strong growth over the longer term. Many of the structural measures that had been pending at the time of the last review have since been implemented. To support us in our efforts and based on the attached economic program, plus the specific targets set out in Tables 1 and 2 attached to this letter, we request an 18-month arrangement under the IMF's Exogenous Shocks Facility (ESF) in the amount of SDR 66.6million (approximately \$100 million), equivalent to 75 percent of our quota to help cover our balance of payments need.

We understand that the requested ESF arrangement will be subject to semi-annual reviews, semi-annual performance criteria, and structural benchmarks, as set out in the attached Tables 1 and 2, and described in more detail in the attached Technical Memorandum of Understanding (TMU). In this regard, we understand that the completion of the first review under the ESF arrangement — which is to be completed no later than May 2009-will require observance of the quantitative performance criteria for end-December 2008 in Table 1 and that completion of the second review - which is to be completed no later than November 2009—will require observance of quantitative performance criteria for end-June 2009, which, in light of the current large uncertainty, will be set at the time of the first review.

We believe that the policies set forth in our economic program are adequate to achieve our objectives, but we are prepared to take all additional measures necessary to achieve these objectives. We will consult with the IMF on the adoption of these measures and in advance of any revision to the policies contained in our economic program, in accordance with the IMF's policies on such consultation. The Kyrgyz government and the National Bank of the Kyrgyz Republic will continue to provide the Fund with the necessary information for assessing progress in implementing our program, as specified in the attached TMU, and will maintain a close policy dialogue with the IMF staff. We authorize the IMF to publish the Letter of Intent and its attachments, as well as the related staff report on the IMF's website following consideration of our request by the IMF's Executive Board.

Sincerely yours,

/s/

Igor Chudinov

Prime Minister of the Kyrgyz Republic
$/ \mathrm{s} /$

Marat Alapaev

Chairman

National Bank of the Kyrgyz Republic 


\section{ATTACHMENT II}

\section{GOVERNMENT OF THE KYRGYZ REPUBLIC \\ AND \\ NATIONAL BANK OF THE KYRgYZ REPUBLIC}

\section{ECONOMiC Program Of THE KYRgYZ REPUbLIC FOR 2008-2009}

\section{INTRODUCTION}

28. The Kyrgyz Republic achieved significant progress in accelerating economic growth and raising living standards during 2005-2007, despite social and political instability in 2005-2006. This was mainly due to the consistent implementation of sound fiscal and financial policies and further progress in structural reforms. As a result, economic growth rebounded to an adequately strong level: in 2007, real GDP growth exceeded 8 percent and excluding gold production growth reached almost 9 percent. The fiscal deficit was reduced to 0.3 percent of GDP, largely reflecting strong revenue performance. The poverty rate declined from 46 percent in 2004 to 35 percent in 2007, with an even stronger reduction in extreme poverty. GDP per capita increased from US\$ 435 in 2004 to US\$ 713 in 2007. Inflation remained at a low level of around 5 percent, until it surged toward the end of 2007 due to rising world food and fuel prices. Also with strong economic growth and firm fiscal discipline, and borrowing only on concessional terms and grant basis, coupled with successful bilateral negotiations for external debt restructuring, the debt burden has eased significantly. Public and publiclyguaranteed external debt declined from 93 percent of GDP in 2003 to 55 percent of GDP in 2007.

29. The international economic environment, however, has deteriorated quickly and substantially since the fall of 2007 and a number of exogenous shocks have hit the Kyrgyz Republic that threaten to undermine our hard-won gains and pose considerable challenges for macroeconomic management. After five years of strong economic growth, the world economy and especially the regional economy are slowing down in the wake of the financial turbulence that started in the summer of 2007 and intensified in recent months. The situation was aggravated by soaring world prices for food and oil products, which increased by over 50 percent between mid-2007 and mid-2008. Since imports account for 100 percent of oil products and natural gas used domestically, for over 55 percent of wheat consumption, and for over 67 percent of vegetable oil consumption, these price shocks had a severe adverse impact on our economy. In addition, water inflows in the Kyrgyz Republic's Toktogul reservoir have been lower than normal. As a result, water levels in the reservoir, which had fallen sharply due to a far greater demand for electricity than usual during the last and exceptionally severe winter, are well below the levels needed to allow for sufficient power generation during the upcoming winter season. Furthermore, an earthquake hit the southern parts of the Kyrgyz Republic in early 
October. The earthquake caused a substantial loss of life and major damage to housing and infrastructure in the remote area surrounding Nura, at the border with Tajikistan and China.

30. This document outlines the economic policies of the Government and the National Bank of the Kyrgyz Republic (NBKR) to address the adverse consequences of these exogenous shocks and to manage the increased risks in the current very difficult and uncertain external environment. These policies also reflect the new economic policy announced by the President of the Kyrgyz Republic in his address on January 10, 2008 and reaffirmed in his address of October 17, 2008.

\section{ADVERSE CONSEQUENCES OF EXOGENOUS SHOCKS}

31. The exogenous shocks threaten to undermine macroeconomic stability and to increase poverty levels through the erosion of real incomes and a reduction in employment opportunities:

- In 2008, economic growth (excluding gold production) is expected to slow to 5 percent. The main causes of this slowdown in growth are reduced domestic demand due to higher prices of food products and services, and lower demand for exports, primarily from Kazakhstan. In addition, credit growth has slowed sharply due to spillover effects from banking sector difficulties in Kazakhstan. Rolling power outages had to be introduced to preserve water in the Toktogul reservoir for use during the coming winter months, further hurting economic activity. The overall rate of economic growth is expected to reach over 7 percent, due to a strong recovery in gold production.

- $\quad$ Higher world prices for food and oil products have led to a surge in inflation, with twelve-month inflation exceeding 30 percent in June 2008. Inflation eased to 26 percent in September, following the recent decline in international commodity prices. But as the higher food and fuel prices have started to feed through into the prices of other goods and services, world prices for food and key energy imports (mainly natural gas) are expected to remain substantially above their 2007 levels, and taking into account the increases in the prices of municipal public transport, electricity, natural gas, and public utilities, inflation will most likely remain high at close to 20 percent at end-2008, above the original 12-15 percent target inflation range defined by the Joint Statement of the Government and the National Bank of the Kyrgyz Republic regarding economic policies for 2008 .

- $\quad$ The current account balance is worsening sharply in 2008 as a consequence of the exogenous shocks. In 2008, due to higher international prices, the (net) cost of importing food and oil products will be almost US\$ 300 million higher than in 2007. In addition, higher import volumes of fuel are needed to avoid major disruptions in the supply of electricity and heating this winter. This is only partially offset by higher gold export receipts. There is a threat that the foreign exchange reserves of the NBKR will fall below 3 months of imports by end- 2008 . 
- The increase in food and energy prices has an adverse affect particularly on lowincome households as a considerable part of their income is spent on basic needs, the costs of which have risen sharply. Notwithstanding the efforts of the Government to provide targeted social assistance to low-income households, we expect a rise in poverty levels, and especially in extreme poverty.

32. Combined, these shocks put food and energy security in the Kyrgyz Republic at serious risk and the outlook is particularly worrisome for the coming winter. Given the shortages of water in the Toktogul reservoir, there is a large risk of major power shortages, which would not only affect economic activity, but also, and more importantly, living conditions. Similarly, while adequate food supplies may be available, basic food items (and energy products) will not be affordable for the low-income households. To avoid a major social and economic crisis, the Government will need to find the means to import sufficient volumes of fuel needed for the additional generation of electricity and heating by the Bishkek heating and power plant, as well as to provide higher levels of financial support to low-income households to ensure that they can meet their basic needs.

\section{ECONOMIC OBJECTIVES AND POLICIES FOR 2008-2009}

\section{A. Objectives}

33. The main objective of the economic policies of the Government and the NBKR is to improve the living standards through strong and sustainable economic growth. A significant and lasting reduction in poverty will require strong economic growth over the medium term, which, in turn, will require the creation of a business-friendly environment and the attraction of sufficient volumes of investment. This is becoming more difficult to achieve because of the recent challenges and threats. In the period ahead, the efforts of the Government and the NBKR will focus on managing the effects on the Kyrgyz economy of the slowdown in regional economic growth and potential spillover effects from the global financial crisis. Policies will focus on achieving the following main goals:

- $\quad$ gradually return inflation to single digits;

- $\quad$ sustain and accelerate economic growth; and

- $\quad$ protect the poorest groups of the population.

34. While working to achieve these objectives, the Government and the NBKR will develop a long-term vision for the Kyrgyz Republic's development and its role and place in the world and in the region. In this context, we are updating the Country Development Strategy (CDS). The CDS for 2007-2010, adopted in 2007, aimed at consolidating the earlier successes and securing further sustained economic growth. It focused on four 
strategic priorities: economic development, governance and transparency in public administration, human capital development, and environmental sustainability. The exogenous shocks that have hit the Kyrgyz Republic, however, have created severe challenges for both the implementation of the CDS and the achievement of its objectives. The update of the CDS will take into account the current challenges and threats, and reflect the new economic policy directions announced by the President in January and October of this year. In addition, we will design a strategy for social and economic development of the Kyrgyz Republic until 2020.

\section{B. Strategy}

35. Sound macroeconomic policies will be critical to achieve our objectives. The sharp increase in inflation poses a particular challenge for monetary policy. Monetary policy will aim at bringing down inflation to single digits, while being responsive to underlying economic conditions. Our exchange rate policy will continue to rely on a managed float regime, with interventions by the central bank aimed at smoothing exchange rate fluctuations.

36. Fiscal policy will need to be supportive of monetary policy, to help reduce inflation, while balancing this with the need to support economic growth and the poor. In order to mitigate inflationary pressures and achieve the inflation targets, we will take measures to contain the overall government budget deficit. Key objectives of our fiscal policy also include ensuring food and energy security, including the full and timely funding of social commitments, and the creation of favorable conditions for economic growth. The budget for 2009 will be based on the Medium Term Fiscal Framework for 2009-2011, which targets to limit the overall budget deficit to an annual average level of about $1 \frac{1}{2}$ percent of GDP, by improving revenue collection and containing spending growth.

37. Fiscal consolidation will also contribute to further reducing the still high levels of external debt. The Government will continue to focus on external debt sustainability, including by considering all opportunities for further debt restructuring and by restricting the contracting of government and government-guaranteed foreign debt to loans that have a grant element of at least 35 percent and that will have a maximum impact on the Kyrgyz Republic's economic development. For the purposes of improving the efficient use of foreign aid, the Government will continue its efforts to improve coordination and interaction with donor countries and international financial organizations.

38. The creation of an environment that favors private sector development and is conducive to attracting higher levels of private, including foreign, investments will also be a priority for reaching our objectives. The Government will therefore continue to work to improve the business and investment environment and to advance structural reforms, including in the energy sector. Developing production and social infrastructure is of 
significant importance for the future of the country, as it will provide the foundation for accelerated economic growth and increased competitiveness through higher labor productivity. Improved infrastructure will have an impact on all sectors of economy and significantly increase the share of value added. Higher labor productivity, diversification and acceleration of exports will be achieved through encouragement of small and medium-size businesses and the development of industries in which the Kyrgyz Republic has proven or potential competitive advantages, particularly in the energy sector, mining, agriculture, processing, and tourism. In this regard, the Kyrgyz Republic will maintain a liberal trade and exchange regime and we will phase out the export duties that were introduced in late 2007 and early 2008. Reforms aimed at financial sector development will help to increase savings and increased volumes of domestic financing for the economy.

39. With the successful implementation of these macroeconomic policies, along with our efforts to further improve the business environment, but against the background of an expected significant slowdown in economic growth in the region, we hope to be able to limit the slowdown in the pace of growth in the Kyrgyz Republic. We project economic growth (excluding gold production) to fall to 3 percent in 2009, before gradually accelerating to 6-7 percent over the medium term, as growth in the region recovers. With gold production expected to increase further in 2009 , overall growth is projected to reach almost 4 percent in 2009. The current account deficit is projected to remain large in 2009, as external demand weakens and remittance inflows are expected to slow, the effects of which are expected to be partially offset by lower oil prices and higher gold exports. As growth in the region recovers, the current account balance is projected to gradually improve in the following years.

40. To ensure that we reach our objectives, we attach a high importance to the effective interaction of ministries and agencies for the purpose of improving coordination of measures in fiscal, monetary, investment, antitrust, and social sector policies. In this connection, we have recently established the Interagency Coordination Council, which is responsible for creating an environment for making coordinated and mutually acceptable policy decisions. The Ministry of Economic Development and Trade will play a central

role in monitoring economic developments and in economic analysis and forecasting and, with the assistance of international organizations, will strengthen its capacity in these areas. Formulating effective policies will also require further improvements in our economic data and we hope to be able to rely on continued technical assistance provided by international donors and organizations, particularly in the areas of national accounts, trade, and government finance statistics.

\section{Monetary and Exchange Rate Policy}

41. Monetary policy will focus on its primary objective, which is to achieve price stability, while also giving consideration to its secondary objective, supporting growth. In 
this regard, the main task of monetary policy in the coming period is therefore to prevent high inflation rates from becoming embedded in the economy, as persistent double-digit inflation will undermine economic growth. The key goal of our monetary and exchange rate policy for 2008-2010 will be to regain control over inflation and to bring it down to about 20 percent by the end of 2008 and further to about 12 percent by the end of 2009 , with a view to returning to single digits thereafter, recognizing there are significant downside risks. The NBKR, in the conduct of its monetary and exchange rate policies, will not be constrained by profitability considerations. The Ministry of Finance will support the NBKR, in accordance with the provisions of the Law on the NBKR, to ensure that the NBKR's capital remains positive.

42. In order to reduce inflation, the NBKR has tightened its monetary policyincluding a sharp increase in interest rates - to mitigate the second-round effects of the increase in food and energy prices and to prevent an upward adjustment in inflation expectations. In the period ahead, the NBKR will carefully manage the need to further reduce inflation with the need to support growth, now that growth is expected to slow. In doing so, the NBKR will continue to manage the money supply through its open-market operations and will set interest rates on the instruments it uses to withdraw excess liquidity as needed. The NBKR will continue to manage liquidity through the issuance of NBKR notes and could also use its deposit facility, while a gradual conversion of nonmarketable government debt in its portfolio into marketable instruments by the Ministry of Finance will allow the NBKR to make greater use of repurchase operations. Coordination between the NBKR and the Ministry of Finance in the areas of liquidity management and the issuance of securities will be further improved.

43. Given the large import content of the consumer basket, the exchange rate can also be a powerful instrument to influence inflation. Therefore, in case of foreign exchange inflows, the NBKR will allow greater exchange rate flexibility to help bring down inflation faster. To the extent that the NBKR purchases foreign exchange to replenish its foreign exchange reserves, it will sterilize the effects on reserve money. In case of downward pressures on the exchange rate, the NBKR intends to support the Som, through unsterilized foreign exchange sales, to limit further inflationary pressures. The NBKR will avoid large reserve losses if the pressures were to persist or intensify, but instead would further tighten its monetary policy. The NBKR and the Government will closely monitor how exchange rate movements impact inflation and affect the competitiveness of domestic producers.

\section{Fiscal Policy}

44. Fiscal policy will aim to strike a balance between curbing inflation pressures and supporting economic growth and the poor. The surge in inflation and the need to ensure food security required revisions to the 2008 State budget, including increased expenditures related to the procurement of grain, fuel, agricultural equipment, length-of- 
service allowances for teachers, salary increases and meal allowances, and funds to clear arrears of local governments to utility companies. The Parliament approved the revised 2008 budget submitted by the Government at end-June. Further revisions have become needed to allow for the import of fuel for electricity and heating generation and additional social support. These additional expenditures will be partially offset by higher revenues, in both the State and Social Fund budgets, as well as by savings on non-priority spending. Thus, the overall budget deficit is expected to remain limited to 1.3 percent of GDP in 2008.

45. The Government will continue mitigating the impact of rising prices on the poor through targeted social assistance. Even though the main social benefits - the Unified Monthly Benefit (UMB) and Monthly Social Benefit (MSB) - have weaknesses in term of targeting, increasing benefit levels and expanding coverage is the most direct and efficient way to help cushion the impact of higher food and energy prices on the poor in the short term. As a first step, the Guaranteed Minimum Consumption Level (GMCL)which is the basis for the UMB and MSB — was raised by Som 25 on January 1, 2008. Further, as of October 2008, the UMB is being topped up by Som 35 per UMB recipient. This top-up of the UMB will initially be funded by a World Bank grant, while other donors may also provide funding. The Government, however, will at least sustain the augmented UMB at the level not less than the combined UMB and the top-up, after the donor resources have been exhausted. Meanwhile, we will also refine the eligibility criteria for the UMB to extend its coverage to deserving-but-not-covered poor households and reduce leakage to non-poor households, to be implemented in 2010. In addition, the value added tax (VAT) rates on grain and flour-which are the most vital items for the poor-have been temporarily reduced to 10 percent and could be reduced further. This will be reassessed, with a view to eventually being reversed, as social benefit programs are strengthened. Budgetary outlays for specific support programs, such as for the provision of food to children, have also been increased in the 2008 budget.

46. A new Tax Code was recently adopted by Parliament and will come into effect on January 1, 2009. To help accelerate growth, the new Tax Code aims to further reduce the tax burden on enterprises and individuals and to streamline the number of different taxes and the tax collection processes, also with a view to reducing the informal economy. The reduction in the VAT rate from 20 percent to 12 percent will bring the tax regime in line with neighboring countries. To offset revenue losses, especially from the reduction in the VAT rate, the new Tax Code introduces a property tax and a new turnover tax that replaces the existing sales, road, and emergency taxes. As it will take time to register property owners and assess property values, it may be necessary, at least in the short term, to take temporary measures to boost revenues and to find additional cuts in nonpriority current spending in order to be able to limit the 2009 overall budget deficit to 1.7 percent of GDP. In this regard, increases in the government sector wage bill will be limited to the real GDP growth rate. 
47. The Government, and notably the Ministry of Finance, will further strengthen the management of public finances:

- $\quad$ One area of focus is aimed at improving Treasury operations and strengthening fiscal accounting and reporting. In the context of the Treasury Modernization program, we will present revisions to the Treasury Law to Parliament to ensure alignment of existing legal framework with new system requirements. Also, a new chart of accounts will be adopted in ministries and budget agencies consistent with the 2001 Government Finance Statistics Manual and, starting in January 2009, budget implementation and reporting will be done on the basis of this new chart of accounts.

- We will furthermore design a development strategy to deepen the market for government securities and increase its liquidity. In this context, the Ministry of Finance will restructure the government securities issuance calendar by introducing the practice of re-opening existing Treasury bill series for sale in subsequent auctions, to reduce the number of outstanding different series of government securities.

- $\quad$ To enable a better assessment of the overall fiscal policy stance, the State budget submitted to Parliament includes a consolidated presentation of the State and Social Fund budgets. Transfers of public resources to the Development Fund and the Kazakh-Kyrgyz Fund will be reflected transparently in the State budget. Thus, Parliament will retain ultimate authority over decisions affecting the use of all public resources. The Development Fund will refrain from borrowing or issuing guarantees to ensure consistency with the Law on Public and Non-Public Debt of the Kyrgyz Republic. Reporting and auditing of the Development Fund will be in line with the provisions in the Law on the Development Fund.

- $\quad$ Revenue administration will be strengthened as well, to streamline tax payment processes and reduce corruption. The new Tax Code will introduce risk-based auditing procedures and no longer contain the provision that each taxpayer should be audited at least once a year. In line with the principle of self-assessment, processing of returns will be purely mechanical, without a numerical review of returns by staff of the State Tax Inspectorate (STI) during filing, to use resources efficiently and reduce interactions between taxpayers and STI staff. Facilities to file electronically will be improved. Any review of tax returns will only be done at the audit stage.

- A new Internal Audit law has already been approved by the Parliament's Economy, Budget and Finance Committee and is expected to be passed by end-2008. This law will govern internal audits of government agencies and institutions and establish the principles and basis for conducting audits in line with international best practice. This will allow for improvements in financial and administrative governance and the use of resources.

- To further help control the wage bill, a civil service reform program will be developed, focusing on payroll policies (covering all employees paid from the budget) and establishing a centralized payroll database. 
- Meanwhile, the budget will continue to refrain from accumulating new arrears on wages, contributions to the Social Fund, transfers to the Social Fund, benefits and allowances to low-income households, equalizing and categorized grants, and electricity bills.

48. Reforming the pension system is a priority of fiscal and social policies. The ultimate goal is a complete transition to a fully funded pension system. To this end, a proper regulatory framework will be created and measures will be taken to promote the establishment of a sufficiently liquid securities and government debt market. One of the measures to raise the level of pension security is the introduction of a fully-funded pillar into the existing pension system and the promotion of voluntary individual pension savings. The reduction in the pension age approved in 2007 has been recently reversed and the Government and the Social Fund will continue their efforts to prevent reductions in the pension age and to introduce a system of flexible retirement age. Improving the administration of contributions and enhancing the legislative framework with a view to securing the financial sustainability of the Social Fund will allow further increases in pension levels. Meanwhile, contributions from the employer will be reallocated to the employee and the redistributed part will be channeled to the funded pension system when this becomes effective by mid-2009 at the earliest.

\section{E. Financial Sector Policies}

49. In the financial sector, the Government and the NBKR will focus on achieving further financial deepening and strengthening financial sector supervision. The level of financial intermediation is still very low in the Kyrgyz Republic, which hampers economic development. Banks offer only a limited menu of financial services and interest rate spreads remain high. Credit to the private sector is still less than 20 percent of GDP, while only about 6 percent of the population has a bank account. Medium and small-size enterprises and many households, particularly in remote areas, have virtually no access to financial services. A greater level of financial intermediation would be beneficial to growth and enhance the effectiveness of monetary policy. The planned privatization of Ayul Bank, which was recently approved by Parliament, will increase access to financial services in remote areas through its wide branch network. We intend to launch a tender for the sale of Ayul Bank towards the end of 2009, depending on conditions in the global financial markets.

50. The Kyrgyz banking sector has so far weathered the spillover effects from the financial sector difficulties in neighboring Kazakhstan well, due to its high capitalization and liquidity. But vigilance remains needed and the NBKR and the Government are prepared to take measures, including the provision of financial support, to ensure the stability of the Kyrgyz banking system. The NBKR is well equipped to provide liquidity support, including through its rediscount facility introduced in February 2008. The existing bank resolution framework allows the NBKR and the Government to take 
appropriate action to address potential solvency problems. The NBKR will continue to strengthen its banking supervision, including through enhanced analysis and inspections, to be able to detect any potential liquidity or solvency problems early on. In this regard, the NBKR will continue efforts to improve its methodology and practices of assessing bank risks, by introducing elements of risk-oriented supervision, including the improvement of early-warning systems, a system of consolidated supervision, and a credit registry at the NBKR for its own supervisory purposes. The NBKR will also further increase the quality of its staff through increased training and by providing an appropriate incentive structure. The minimum capital requirement for existing commercial banks will be doubled to Som 200 million, effective end-2010, to ensure stable and sustained functioning of commercial banks and to maintain the capital base necessary to cover the risks. In the light of government policy to promote competing financing systems and to expand the range of banking services, the NBKR will continue to create conditions for promoting financing in accordance with Islamic finance principles.

51. The Kyrgyz Parliament also recently approved the introduction of a deposit insurance scheme, which will help to further improve confidence in the banking system. The scheme will come into effect in 2010, or as soon as the Deposit Insurance Fund reaches its target level (with the government making its contribution no later than August 2009). Only banks that meet all prudential requirements will be allowed to participate in the deposit insurance scheme. Effective cooperation between the National Bank and the Deposit Protection Agency will help protect the interests of depositors in the event of bank insolvency.

52. On the lending side, creditor rights, creditor information, collateral arrangements, property registration, and the use of international accounting standards will be improved. Already, the pledge law and civil code have been amended to allow for out-of-court procedures to seize collateral. Additional changes have been made to the Civil Code to allow banks to share borrower information with third parties, including credit bureaus. In order to reduce the financial costs for potential borrowers and the time it takes to obtain loans, the Government will take further action to improve pledge registration procedures by the end of 2009 in connection with the mandatory registration of real estate property titles. Pledge registration procedures for lending purposes should be convenient and affordable for households, the bulk of which reside in rural areas and are low-income households. The NBKR will also take measures aimed at protecting the consumers of banking services and ensuring compliance of banks with market discipline when circulating information regarding fees and commissions for banking services.

53. The long-term strategic objectives of payments system reform in the Kyrgyz Republic are aimed at supporting the secure and uninterrupted operation of payments systems in all sectors of the economy in order to ensure a stable functioning of financial markets and preserve confidence in the national currency. The NBKR will continue to 
promote effective, reliable, and inexpensive methods of providing payment and financial services to consumers and to bring the payments system in line with international standards. In this regard, the NBKR intends to secure the strategic objectives of reforming the nation's payments system by promoting: (i) a wider range of retail payments services offered by financial and lending institutions by developing a system for batch clearing of retail and regular payments and assisting the commercial banks in developing the infrastructure for settlements with bank-issued payments cards; (ii) improved access to banking services in the regions by assisting in the development of the banking and payments system infrastructure; (iii) an environment for improved effectiveness of payments processing for financial market transactions by integrating the gross settlements system and operators of exchange floors; and (iv) improvement of the regulatory framework along with the introduction of new payments instruments and systems. Furthermore, for the purpose of developing the financial sector, the NBKR will continue the project for the acquisition of automated trading systems required to enhance the domestic financial markets infrastructure, including of the foreign exchange, government securities, and money markets.

\section{F. Business and investment environment}

54. Strong economic growth requires a business-friendly environment and adequate infrastructure. The Government sees the creation of an environment conducive to private sector growth and attracting investment as a top priority. We will focus special attention on attracting foreign direct investment into sectors which have been identified as showing considerable potential: power generation, mining, tourism, telecommunications, agriculture and processing, and banking. More generally, the Government and the NBKR will continue to identify and implement reforms to make it easier to do business in the Kyrgyz Republic and improve the country's ranking in the Doing Business surveys. A one-stop window has been established to ease business registration and investor protection has been strengthened. A one-stop shop was also introduced for construction permits. Key areas for additional reform will be to further simplify administrative procedures (including tax, customs, and trade procedures) and to reduce corruption. Particular emphasis will be paid to ensure the transparency of supervisory activities and minimizing the number of audits in order to eliminate corruption among government officials. In this regard, we will develop an automated system for processing audit-related data and a rapid response policy vis-à-vis complaints from private sector businesses. This will allow us to eliminate groundless audits, avoid duplication of functions for supervisory agencies and protect the rights of entrepreneurs. All draft regulations which have a bearing on private businesses will be subject to a mandatory review process to assess their regulatory impact. A new Program for Informal Sector Legalization for 20082010 will be developed to draw informal activity into the formal sector.

55. To ensure that businesses and individuals can rely on an uninterrupted energy supply, the Government has adopted a National Energy Program. As part of this program 
some segments of the energy sector will be made available for privatization, while private entry will also be allowed in power generation. The program furthermore aims to improve the energy sector's financial soundness by gradually raising utility tariffs. A feasibility study will be conducted for the Kambar-Ata 1 hydropower project and the Government will seek private participation and support from international donors for the development of this regionally critical project. 
Table 1. Kyrgyz Republic: Quantitative Program Targets

(In millions of soms, unless otherwise indicated; eop)

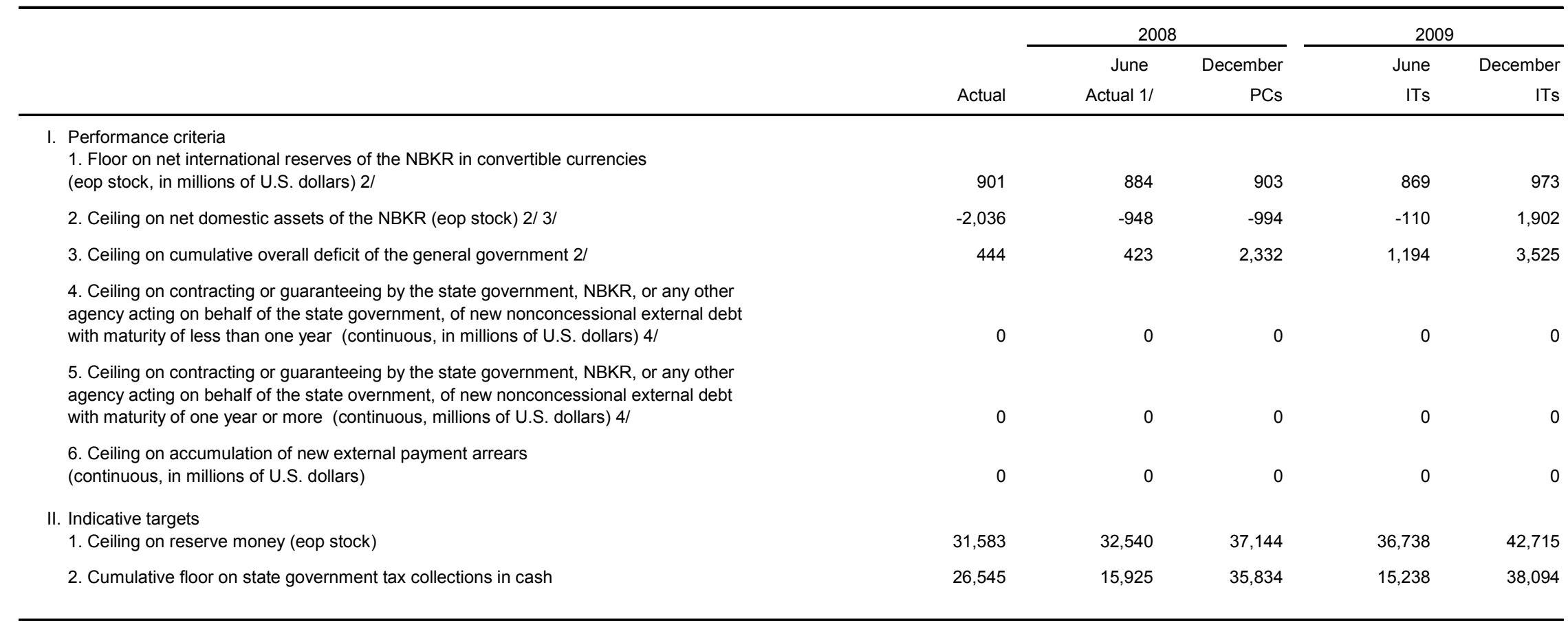

Sources: Kyrgyz authorities; and Fund staff estimates and projections.

1/ Preliminary data for fiscal outcome.

2/ These targets are subject to adjustment in case of changes in flows of external budget financing by bilateral and multilateral donors as defined in the TMU.

3/ The target excludes medium- and long-term central bank liabilities (i.e., the loan by the Eximbank of Turkey and the EBRD/IDA enterprise loan channeled through the NBKR)

4/ External debt contracted or guaranteed other than with a grant element equivalent to 35 percent or more, calculated using a discount rate based on the OECD commercial

interest reference rates. Excludes borrowing from the IMF. 


\section{Table 2. Kyrgyz Republic: Structural Measures}

I. Structural benchmarks for continuous implementation

- $\quad$ The Ministry of Finance and the National Bank of the Kyrgyz Republic to exchange and discuss projected financial operations, including state budget spending and revenue forecasts and securities and notes issuance plans on at least a bi-weekly basis.

- $\quad$ Reflect the transfers of public resources to the Development Fund and the KazakhKyrgyz Fund transparently in the State budget, while the Development Fund refrains from unsecured borrowing and issuing guarantees to guarantee consistency with the law on Public and Non-Public Debt of the Kyrgyz Republic.

II. Structural benchmarks for 2008

- $\quad$ Adopt the Resolution on Decision Making Procedure for Measures for Rehabilitation of Banks of Systemic Importance, identifying the roles of the NBKR and the Ministry of Finance in the bank resolution process (end-December, 2008).

- $\quad$ Submit to Parliament amendments to the Law on the NBKR to: (i) increase its statutory capital to at least Som 1 billion; and (ii) ensure that net unrealized price and exchange rate valuation gains are not reflected in the NBKR's income statement, while net unrealized price and exchange rate valuation losses are reflected in the NBKR's income statement at the end of the financial year, in line with international central bank best practice. This provision shall be reflected in the NBKR's financial reporting (endDecember 2008).

III. Structural benchmarks for 2009

- $\quad$ Conduct a review, with the assistance of the IMF, of the pilot project at the stock market selected to become the platform for the government securities market to assess whether arrangements meet public interests, including confidentiality and transparency concerns, and international best practices. This review will examine: (i) information sharing and decision making for public debt auctions; (ii) governance structures; and (iii) settlement and custody arrangements (end-March, 2009).

- $\quad$ Convert the off-balance government security held by the NBKR into an interest-bearing security that can be placed on-balance and ensure that the NBKR's capital is at least equal to its statutory minimum (end-December, 2009). 


\section{ATtachment III. TeChnical Memorandum OF Understanding}

\section{INTRODUCTION}

1. The Kyrgyz Republic's performance during the period October 1, 2008December 31, 2009 under the 18-month arrangement under the Exogenous Shocks Facility will be assessed by the IMF on the basis of the observance of quantitative performance criteria and structural benchmarks. This memorandum and its attached tables define the quantitative performance criteria and indicative targets under the ESF-supported program. It reflects the Economic Program of the Kyrgyz authorities as attached to their Letter of Intent dated November 25, 2008.

2. The program exchange rate of the Kyrgyz som to the U.S. dollar is set at som $38.2101=\$ 1$. The corresponding cross exchange rates and program gold price for the duration of the program are provided in Table 7.

3. Central government and republican government are synonymous in this memorandum. The State budget comprises central and local government budgets. The general government budget comprises the State and Social Fund budgets.

\section{Quantitative Performance Criteria}

4. The quantitative targets (i.e., quantitative performance criteria for end-December 2008, and quantitative benchmarks for end-June and end-December 2009) presented in Table 1 of the economic program attached to the letter, dated November 25, 2008, are defined below.

\section{A. Floor on net international reserves of the National Bank of the Kyrgyz Republic (NBKR) in convertible currencies}

5. The program contains a floor on the stock of net international reserves of the NBKR in convertible currencies. This floor will be calculated as the difference between total international reserve assets and total international reserve liabilities of the NBKR in convertible currencies.

6. Total international reserve assets of the NBKR are defined as the NBKR holdings of monetary gold, holdings of SDRs, any reserve position in the IMF, and any holdings of convertible foreign currencies in cash or with foreign banks, and debt instruments (including accrued interest) issued by nonresidents that are liquid. Amounts pledged as collateral or in swaps or otherwise encumbered, capital subscriptions in foreign financial institutions, and illiquid assets of the NBKR are excluded. Also excluded are net forward positions, defined as the difference between the face value of foreign currency denominated NBKR off-balance sheet claims on nonresidents and foreign currency obligations to both residents and nonresidents. For program monitoring purposes, international reserve assets shall be valued at program exchange rates and gold prices. Total international reserve liabilities of the NBKR in convertible currencies are defined as outstanding liabilities to the IMF and other convertible currency liabilities to nonresidents with an original maturity of up to and 
including one year. For program monitoring purposes, total international reserve liabilities will be valued at the program exchange rates as described in paragraph 2 above.

7. Thus calculated, the stock of net international reserves in convertible currencies amounted to \$901 million at December 31, 2007 and \$884 million at June 30, 2008. The program floors on the NIR of the NBKR in convertible currencies are reported in Table 1 below.

Table 1. Floors on NIR of the NBKR in Convertible Currencies $1 /$

(In millions of U.S. dollars)

\begin{tabular}{ll}
\hline December 31, 2008 (performance criterion) & 903 \\
June 30, 2009 (indicative target) & 869 \\
December 31, 2009 (indicative target) & 973 \\
\hline
\end{tabular}

1/ End-of-period stocks.

8. The NBKR's net foreign assets consist of net international reserves plus other net foreign assets, including the medium- and long-term foreign obligations of the NBKR, other net claims on CIS countries, amounts pledged as collateral or in swaps or otherwise encumbered, net forward positions, capital subscriptions in foreign financial institutions, and illiquid assets. For program monitoring purposes, the other net foreign assets will also be valued at the program exchange rates.

\section{B. Ceiling on the net domestic assets of the NBKR}

9. Net domestic assets of the NBKR are defined as reserve money of the NBKR (defined below), minus the NBKR's net foreign assets as defined above, minus the counterpart of the loan by the Eximbank of Turkey and the counterpart of the EBRD and IDA enterprise loans. Items in foreign currencies will be valued at the program exchange rates.

\section{NDA=RM-NFA-Turkish Loan-EBRD-IDA Enterprise Loan}

10. Thus defined, the NBKR's net domestic assets consist of: (a) gross credit to the general government from the NBKR, minus deposits of the general government with the NBKR, minus the counterpart of the loan by the Eximbank of Turkey; (b) gross credit to domestic banks by the NBKR, minus the counterpart of the EBRD and IDA enterprise loans; and (c) all other net assets of the NBKR (other items net). Thus defined, the stock of the NBKR's net domestic assets amounted to minus Som 2,036 million on December 31, 2007 and minus Som 948 on June 30, 2008. 
11. The program ceilings on the NDA of the NBKR are reported in Table 2 below.

Table 2. Ceilings on the NDA of the NBKR $1 /$

(In millions of soms)

\begin{tabular}{ll}
\hline December 31, 2008 (performance criterion) & -994 \\
June 30, 2009 (indicative target) & -110 \\
December 31, 2009 (indicative target) & 1902 \\
\hline
\end{tabular}

1/ End-of-period stocks.

\section{Ceiling on the cumulative overall deficit of the general government}

12. The overall deficit of the general government is defined as the sum of: (a) the change in the stock of net claims of the domestic banking system and nonfinancial institutionsincluding state-owned enterprises and public companies - and households on the general government; (b) the change in the stock of net claims of the foreign banking system and nonfinancial institutions and households on the general government; (c) net privatization receipts including from new Centerra share sales; (d) net foreign loans disbursed to the state government for budgetary support; and (e) net foreign loans disbursed to the general government for project financing. The fiscal balance will be measured at the program exchange rates, unless foreign currency-denominated assets or liabilities are converted into domestic currency upon receipt or accrual.

13. The change in the stock of net claims of the domestic banking system on the general government is defined as the change in the stock of the banking system claims on the general government, less the change in the stock of all deposits of the general government with the banking system. The claims of the banking system on the general government include: (a) bank loans to the general government; (b) securities or bills issued by the general government held by banks, with the exception of those issued in relation with bank rescue operations; and (c) overdrafts on the current accounts of the general government with banks.

14. The program ceilings on the cumulative overall deficit of the general government are reported in Table 3 below. The ceiling on the overall budget deficit of the general government will be adjusted upward to the full extent that concessional foreign loans with a grant element of at least 35 percent disbursed to the budget are higher than the amounts given in Table 4. The ceiling on the overall budget deficit of the general government will also be adjusted upward to the full extent that program grants received are less than the amounts given in table 4 . The ceiling on the overall budget deficit of the general government will be adjusted downward to the full extent that disbursements of PIP loans are less than the amounts given in Table 4. 
Table 3. Ceilings on the Overall Budget Deficit of the General Government 1/

$$
\text { (In millions of soms) }
$$

\begin{tabular}{lc}
\hline & Overall Deficit \\
\hline December 31, 2008 (performance criteria) & 2,332 \\
June 30, 2009 (indicative target) & 1,194 \\
December 31, 2009 (indicative target) & 3,525 \\
\hline
\end{tabular}

Table 4. Projected Budget Support (millions of US dollars)

\begin{tabular}{|c|c|c|c|}
\hline & 2008 & Jun-09 & Dec-09 \\
\hline Program grants & 45.7 & 20.7 & 41.5 \\
\hline WB & 2.5 & 3.0 & 3.0 \\
\hline EU & 13.0 & 7.9 & 18.9 \\
\hline SWAP (including WB) & 17.6 & 9.8 & 19.6 \\
\hline Other donors & 12.5 & 0.0 & 0.0 \\
\hline PIP grants & 76.4 & 41.6 & 97.2 \\
\hline Asian Development Bank & 16.3 & 13.3 & 31.0 \\
\hline World Bank & 34.6 & 19.9 & 46.5 \\
\hline $\mathrm{KfW}$ & 9.7 & 5.2 & 12.2 \\
\hline Switzerland & 6.3 & 1.6 & 3.8 \\
\hline DFID & 9.5 & 1.6 & 3.8 \\
\hline Program loans (BOP support) & 22.2 & 3.0 & 3.0 \\
\hline WB & 14.4 & 3.0 & 3.0 \\
\hline $\mathrm{ADB}$ & 7.8 & 0.0 & 0.0 \\
\hline Other donors & 0.0 & 0.0 & 0.0 \\
\hline PIP loans & 54.6 & 39.1 & 89.4 \\
\hline ADB & 28.6 & 12.0 & 27.5 \\
\hline WB & 10.2 & 5.1 & 11.7 \\
\hline Islamic Development Bank & 10.0 & 5.6 & 12.8 \\
\hline KFW & 5.8 & 7.6 & 17.4 \\
\hline China & 0.0 & 8.8 & 20.0 \\
\hline
\end{tabular}




\section{Ceilings on contracting or guaranteeing of new external debt by the state government of the Kyrgyz Republic or the NBKR or any other agency acting on behalf of the state government}

15. In connection with the contracting or guaranteeing of external debt by the state government of the Kyrgyz Republic, the NBKR, or any other agency acting on behalf of the state government of the Kyrgyz Republic, "debt" is understood to have the meaning set out in point 9 of the Guidelines on Performance Criteria with respect to External Debt in Fund arrangements (Decision No. 12274-00/85, dated August 24, 2000). ${ }^{2}$

16. External debt ceilings apply to (a) the contracting or guaranteeing of new nonconcessional short term external debt (i.e. external debt with an original maturity of less than one year and grant element less that 35 percent, except normal import-related credits and NBKR reserve liabilities); and (b) contracting or guaranteeing of nonconcessional mediumand long-term external debt (i.e., external debt with an original maturity of one year or more and with grant element of less that 35 percent). Disbursements by the Fund are excluded from the ceilings on external debt. Also excluded from these external debt ceilings is the contracting or guaranteeing of new external debt that constitutes a rescheduling or refinancing of existing external debt at terms more favorable to the debtor. The limit on the contracting or guaranteeing of short-term external debt is zero on a continuous basis throughout the period of the arrangement. The limit on the contracting or guaranteeing of medium- and long-term nonconcessional external debt is also zero and on a continuous basis throughout the period of the arrangement, as specified in Table 1 of the economic program of the authorities.

\footnotetext{
${ }^{2}$ Debt is understood to mean a current, i.e., not contingent, liability, created under a contractual arrangement through the provision of value in the form of assets (including currency) or services, and which requires the obligor to make one or more payments in the form of assets (including currency) or services, at some future point(s) in time; these payments will discharge the principal and/or interest liabilities incurred under the contract. Debt can take a number of forms, the primary ones being as follows: (a) loans, i.e., advances of money to the obligor by the lender made on the basis of an undertaking that the obligor will repay the funds in the future (including deposits, bonds, debentures, commercial loans and buyers' credits) and temporary exchanges of assets that are equivalent to fully collateralized loans under which the obligor is required to repay the funds, and usually pay interest, by repurchasing the collateral from the buyer in the future (such as repurchase agreements and official swap arrangements); (b) suppliers' credits, i.e., contracts where the supplier permits the obligor to defer payments until some time after the date on which the goods are delivered or services are provided; and (c) leases, i.e., arrangements under which property is provided which the lessee has the right to use for one or more specified period(s) of time that are usually shorter than the total expected service life of the property, while the lessor retains the title to the property. For the purpose of the guideline, the debt is the present value (at the inception of the lease) of all lease payments expected to be made during the period of the agreement excluding those payments that cover the operation, repair or maintenance of the property. Under the above definition of debt, arrears, penalties, and judicially awarded damages arising from the failure to make payment under a contractual obligation that constitutes debt are debt. Failure to make payment on an obligation that is not considered debt under this definition (e.g., payment on delivery) will not give rise to debt.
} 
17. For the purposes of the ceiling on contracting and guaranteeing new external debt, any other agency acting on behalf of the state government will in particular include the Development Fund and the Kazakh-Kyrgyz Fund as well as all nonfinancial public enterprises with share capitals of not less than Kyrgyz som 100 million in which the state government holds at least 51 percent of the share capital.

\section{E. Ceiling on accumulation of new external payments arrears}

18. For the purposes of the program, external payment arrears will consist of all debt-service obligations (i.e., payments of principal or interest) arising in respect of any debt contracted or guaranteed or assumed by the state government of the Kyrgyz Republic, or the NBKR, or any agency acting on behalf of the state government of the Kyrgyz Republic since the Kyrgyz Republic's independence, including, without limitations, unpaid penalties, interest charges or judicially awarded damages associated with these arrears owed by the state government of the Kyrgyz Republic, or the NBKR, or any agency acting on behalf of the state government of the Kyrgyz Republic. The ceiling on new external payment arrears shall apply on a continuous basis throughout the period of the ESF arrangement.

\section{INDICATIVE TARGETS}

\section{A. Ceiling on reserve money}

19. For the purposes of the program, reserve money consists of currency issued by the NBKR and balances on commercial banks' correspondent accounts with the NBKR. The indicative program limits are reported in Table 5 below.

Table 5. Ceilings on Reserve Money $1 /$

(In millions of soms)

\begin{tabular}{ll}
\hline December 31, 2008 (indicative target) & 37,144 \\
June 30, 2009 (indicative target) & 36,738 \\
December 31, 2009 (indicative target) & 42,715 \\
\hline
\end{tabular}

1/ End-of-period stocks.

\section{B. Cumulative floor on state government tax collections in cash}

20. Tax collections in cash correspond to the line "Tax Receipts" in the Treasury Report and comprise the following categories: tax on income and profits; taxes on goods and services; specific taxes on services; turnover taxes; taxes on property; taxes on international trade; and other taxes. Cumulative tax collections in cash include collections of tax arrears but exclude tax offsets.

21. The program floors for the cumulative state government cash tax collection are reported in Table 6 below. 
Table 6. Floors on State Government Cash Tax Collections 1/

(In millions of soms )

\begin{tabular}{ll}
\hline December 31, 2008 (performance criteria) & 35,834 \\
June 30, 2009 (indicative target) & 15,238 \\
December 31, 2009 (indicative target) & 38,094 \\
\hline
\end{tabular}

$1 /$ Cumulative from the beginning of the calendar year.

\section{Reporting Requirements Under the Program}

22. The government and the NBKR will provide the Fund with the necessary economic and financial statistical data to monitor economic developments and the quantitative targets. In particular, the government and the NBKR will provide the following specific information: ${ }^{3}$

\section{A. The balance sheet of the NBKR}

23. The NBKR will provide to the Fund its balance sheet on a daily basis. The information provided will clearly identify the following items in the definitions specified above: the gross foreign assets and liabilities of the NBKR, decomposed by currency and instrument for the assets and by currency and creditor for the liabilities (decomposition provided on a monthly basis); the net foreign assets of the NBKR; the net international reserves of the NBKR; medium- and long-term liabilities; the net domestic assets of the NBKR; net credit from the NBKR to the general government; net credit from the NBKR to commercial banks; the balance of unused PIP funds held in the NBKR; other items net; and reserve money. The balance sheet will be provided valued at the actual exchange rate as well as according to the valuation applied under the program, as specified in Section I. The above information will be provided to the IMF Resident Representative and/or transmitted by email to the Fund.

\section{B. Monetary survey}

24. Monthly banking system data, in the form of a monetary survey, will be reported to the Fund by the NBKR within 14 days of the end of the month. The information provided will clearly identify the following items: net foreign assets and net domestic assets of the banking system, medium- and long-term liabilities, net credit from the banking system to the general government, financing provided to the rest of the economy, other items net, and

\footnotetext{
${ }^{3}$ Any correction or revisions to data previously reported should be clearly indicated and documented along with the reasons for revision.
} 
broad money. The monetary survey will be provided valued at the actual exchange rate as well as according to the valuation applied under the program, as specified in Section I.

25. The NBKR will provide monthly data to the Fund within seven days after the end of the month on the amount of holdings of treasury bills, GKOs, state obligations, state bonds, and other securities issued by the state government, differentiated by the following categories of holders: the NBKR, resident banks, resident nonbanks, and nonresidents. The information will be provided in both the book (nominal) value and the actual value, where applicable.

\section{International reserves and key financial indicators}

26. The NBKR will provide detailed monthly data within 14 days from the end of the month on the composition of both its gross and net international reserves in convertible currencies and holdings of monetary gold. These data will be provided at two alternative sets of the exchange rates and the gold price: first, at those used to derive the NFA position in the NBKR accounts; second, at those specified in the program (Section I). In addition, weekly reports should be sent to the Fund on (a) exchange rates (including the official and interbank exchange rates), foreign exchange interbank market turnover, and the volume of NBKR foreign exchange sales and purchases in the interbank market and with other parties, on a daily basis; and (b) treasury bill yields and the amount of treasury bill sales and redemptions on a weekly basis every Monday. On the 25th day of the month following the reference month, the NBKR will provide indicators of financial soundness of the banking system, including the ratios of regulatory capital to risk-weighted assets, nonperforming loans to total loans, and return on equity, as well as data on bank deposit and lending rates by maturity.

\section{External debt}

27. The Ministry of Finance, together with the NBKR, will provide monthly information on the disbursements, principal and interest payment - both actual and falling due; on contracting and guaranteeing of medium- and long-term external loans by the state government and the NBKR; and any stock of outstanding arrears on external debt service payments within 21 days of the end of each month. In addition, the Ministry of Finance will report the total amount of outstanding government guarantees and external arrears on a monthly basis. While the NBKR will provide the debt service payment data on private debt, the ministry of finance will provide data on debt service on public and publicly guaranteed loans.

\section{E. Budgetary and extra budgetary data}

28. In addition to the monthly treasury report, the Social Fund will report monthly on its operations. This information will be provided to the Fund staff within 26 days from the end of each reference month. The Ministry of Finance will also provide monthly reports on the disbursements and use under the public investment program and budgetary grants with a one-month time lag. The Development Fund will also submit a monthly report including its balance sheet and its deposits in the NBKR and the rest of the financial system. 


\section{F. Balance of payments data}

29. The NBKR will provide current account and capital account data, including data on foreign trade, services, official and private transfers, foreign investment, and disbursements of public and private loans, on a quarterly basis, with at most a two-month lag. The NBKR will also provide monthly foreign trade data with a two-month lag.

\section{G. Other general economic information}

30. The National Statistics Committee will notify the Fund of the monthly Consumer Price Index by category by the 15 th business day of the following month, and convey quarterly GDP estimates within two months of the end of each quarter. 
Table 7. Program Cross Exchange Rates

\begin{tabular}{llrr}
\hline & Currency Names & Currency/US\$ & US\$/ Currency \\
\hline & & & \\
AUD & Australian dollar & 0.6329 & 1.5800 \\
AZN & Azerbaidjani manat & 0.8111 & 1.2329 \\
BYR & Belarusian ruble & $2,115.0282$ & 0.0005 \\
CAD & Canadian dollar & 0.7823 & 1.2783 \\
CHF & Swiss franc & 0.8539 & 1.1711 \\
CNY & Chinese yuan & 6.8488 & 0.1460 \\
CZK & Czech crown & 20.1903 & 0.0495 \\
DKK & Danish krone & 5.9253 & 0.1688 \\
EEK & Estonian kroon & 12.4228 & 0.0805 \\
EUR & Euro & 0.7940 & 1.2595 \\
GBP & UK pound sterling & 0.6392 & 1.5645 \\
HUF & Hungarian forint & 223.3072 & 0.0045 \\
INR & Indian rupee & 49.9478 & 0.0200 \\
JPY & Japanese yen & 93.9908 & 0.0106 \\
KRW & South Korean won & $1,420.4498$ & 0.0007 \\
KZT & Kazakh tenge & 119.7809 & 0.0083 \\
LTL & Lithuanian litas & 2.6952 & 0.3710 \\
LVL & Latvian lats & 0.5500 & 1.8182 \\
MDL & Moldavian lei & 10.3176 & 0.0969 \\
NOK & Norwegian krone & 7.0535 & 0.1418 \\
NZD & New Zealand dollar & 0.5730 & 1.7452 \\
PKR & Pakistani rupee & 81.3154 & 0.0123 \\
RUR & Russian ruble & 27.0591 & 0.0370 \\
SDR & SDR & 0.6700 & 1.4925 \\
SEK & Swedish krona & 7.9594 & 0.1256 \\
TJS & Tajik somoni & 3.4026 & 0.2939 \\
TRY & Turkish lira & 0.5875 & 1.7020 \\
UAH & Ukrainian hryvnia & 0.1942 \\
UZS & Uzbek sum & 5.1500 & 0.0007 \\
XAU & Gold (\$/troy ounce) & \\
& & 692.5000 & \\
\hline & & & \\
\end{tabular}


INTERNATIONAL MONETARY FUND

KYRGYZ REPUBLIC

Informational Annex

November 26, 2008

Contents

\section{Annexes}

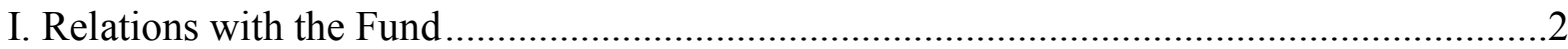

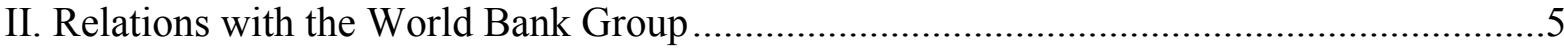

III. Relations with the Asian Development Bank (ADB) ...............................................

IV. Relations with the European Bank for Reconstruction and Development (EBRD).........10

V. Technical Assistance Provided by the Fund ............................................................... 12

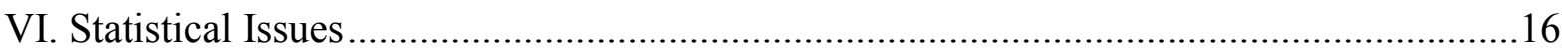




\section{ANNEX I. KYRGYZ REPUBLIC-RELATIONS WITH THE FUND}

(October 31, 2008)

I. Membership Status: Joined: 05/08/1992; Article VIII

II. General Resources Account

Quota

Fund Holdings of Currency

Reserve Position

III. SDR Department

Holdings

IV. Outstanding Purchases and Loans

PRGF Arrangements
SDR Million

88.80

88.80

0.00

SDR Million

20.49

SDR Million

91.34
Percent of

Quota

100.00

100.00

0.01

Percent of

Allocation

N/A

Percent of

Quota

102.86

V. Latest Financial Arrangements

\begin{tabular}{|c|c|c|c|c|}
\hline Type & Approval & Expiration & $\begin{array}{c}\text { Amount } \\
\text { Approved } \\
\text { (SDR Million) }\end{array}$ & $\begin{array}{c}\text { Amount } \\
\text { Drawn } \\
\text { (SDR Million) }\end{array}$ \\
\hline PRGF & $03 / 15 / 2005$ & $05 / 31 / 2008$ & 17.76 & 17.76 \\
\hline PRGF & $12 / 06 / 2001$ & $03 / 14 / 2005$ & 73.40 & 73.40 \\
\hline PRGF & $06 / 26 / 1998$ & $07 / 25 / 2001$ & 73.38 & 44.69 \\
\hline
\end{tabular}

VI. Projected Payments to Fund

(SDR million; based on existing use of resources and present holdings of SDRs)

\begin{tabular}{lrrrrr}
\hline & \multicolumn{5}{c}{ Forthcoming } \\
\cline { 2 - 6 } & 2008 & 2009 & 2010 & 2011 & 2012 \\
\hline Principal & 1.17 & 14.73 & 16.24 & 15.31 & 13.48 \\
Charges/Interest & 0.23 & 0.41 & 0.33 & 0.25 & 0.18 \\
Total & 1.41 & 15.13 & 16.56 & 15.56 & 13.65 \\
\hline
\end{tabular}

\section{Status of HIPC and MDRI Assistance}

The Executive Board considered the preliminary HIPC document on October 13, 2006. However, in 2007, the authorities have decided not to avail themselves of HIPC and MDRI 
assistance. The updated LIC DSA shows that the Kyrgyz Republic does not qualify for HIPC debt relief.

\section{Safeguards Assessments}

Under the Fund's safeguards assessments policy, the National Bank of the Kyrgyz Republic (NBKR) is subject to an update assessment in conjunction with an upcoming arrangement under the high-access component of the Exogenous Shocks Facility. The update assessment is underway. Previous assessments were completed in October 2005 and in January 2002. The 2005 assessment found that the NBKR's safeguards framework had been strengthened, but recommended further steps to solidify progress achieved. In particular, the assessment proposed improving oversight of the audit processes and internal control systems by establishing an audit committee, and further strengthening of the internal audit function.

\section{Exchange Rate Arrangements}

The currency of the Kyrgyz Republic has been the som (100 tyiyn =1 som) since May 15, 1993. The National Bank of the Kyrgyz Republic (NBKR) participates and intervenes in the interbank foreign exchange market to limit exchange rate volatility as necessary. Effective March 1, 2007, the exchange rate arrangement has been reclassified to a conventional pegged arrangement from managed floating with no predetermined path for the exchange rate. During September-November, 2007 the som appreciated by about 10 percent against the U.S. dollar. Thus, effective October 1, 2007, the exchange rate arrangement has been reclassified to managed floating with no predetermined path for the exchange rate. The NBKR publishes daily the exchange rate of the som in terms of the U.S. dollar, which is determined in the interbank foreign exchange market. The official exchange rate of the som against the dollar is defined as the weighted average of the exchange rates of the interbank foreign exchange market transactions for the week.

The Kyrgyz Republic maintains an exchange system that is free of restrictions on the making of payments and transfers for current international transactions, except for exchange restrictions maintained for security reasons relating to the restriction of financial transactions and the freeze of accounts of certain individuals or organizations associated with terrorism pursuant to (i) relevant UN Security Council resolutions and (ii) the list of current terrorist organizations designated by the U.S. Secretary of State. The authorities have notified these measures to the Fund pursuant to Executive Board decision No. 144-(52/51) in May 2007.

\section{Article IV Consultations}

The Kyrgyz Republic is on the 24-month consultation cycle. The last Article IV consultation discussions were held in August 2006 and were completed by the Executive Board in November 2006 (see Country Report No. 07/135). 


\section{FSAP Participation and ROSC Assessment}

An FSAP update mission in October 2006 reviewed progress since the 2002 assessment, and the Board considered the Financial System Stability Assessment (FSSA) along with the $4^{\text {th }}$ PRGF review in May 2007. A fiscal ROSC mission was held in March 2001 and the ROSC Fiscal Transparency Module was published on March 13, 2002. A data ROSC mission was held in November 2002 and the ROSC Data Module was published in November 2003. A fiscal ROSC reassessment was held in September 2007.

\section{Resident Representative}

The seventh resident representative of the Fund in the Kyrgyz Republic, Mr. McHugh, took up his post in Bishkek in late September 2006. 


\section{ANNEX II. KYRGYZ REPUBLIC-RELATIONS WITH THE WORLD BANK GROUP}

(As of November 1, 2008)

The new Joint Country Support Strategy (JCSS) covering FY07-10 was approved by the Board on June 19, 2007. The WB Country team jointly with four development partners (ADB, DFID, SWISS, and UN Agencies) developed JCSS to support implementation of the Country Development Strategy. The JCSS is closely aligned with the Government's development goals. The strategic choices agreed among the development partners are: (i) focus on areas where support for reforms is already embedded; (ii) mitigate risks associated with high indebtedness and growth volatility; (iii) focus on basic reforms, as opposed to complex operations; and (iv) focus on economic and sector work and capacity building.

The World Bank Group Program within JCSS. The WBG program contributes to the strategic goals outlined in the JCSS, with a particular focus on supporting the Government's efforts to improve the environment for business and economic growth and improve the quality of and access to basic services (health, education, water and sanitation). The WBG strategy emphasizes greater selectivity given the limited IDA resources and limitations placed on the Government's own public investment program. Building on lessons learned, and in line with the principles of the JCSS, the Bank Program will build on successful results achieved in first generation projects, target activities where the Bank can show visible results to the population, and leverage our lending and analytical work to attract financing from other development partners. The proposed annual allocation for the Kyrgyz Republic under JCSS 2007-2010 is about \$30 million U.S. dollars.

The JCSS progress report is due in May 2009, and the Country Office has initiated the discussion with other development partners.

IDA allocation: The World Bank has upgraded the status of the Kyrgyz Republic under IDA-5 program. from 'red light' to 'yellow light,' which means that the country is now under moderate risk of debt distress, assessed based on the latest WB/IMF debt sustainability analysis (DSA). The Kyrgyz Republic is assessed to be at moderate risk of debt distress because, starting in 2007, all external debt indicators in the baseline are projected to stay below the indicative threshold over the DSA horizon. Therefore, future IDA allocations for the Kyrgyz Republic will be on a $45 \%$ grant $/ 55 \%$ credit basis.

The total IDA-15 envelope for the Kyrgyz Republic is 79.7 million USD; final FY09 allocation is 25.6 million USD, and indicative FY10 and FY11 allocations are 26.7 and 27.4 million USD correspondingly

IDA Portfolio As of September 2008 active World Bank portfolio in the Kyrgyz Republic comprise 19 operations with total commitments of US\$ 214 million of which US\$108.4 million remains to be disbursed. In addition to 19 projects financed by IDA, the 
World Bank manages EFT-FTA funds and donors TFs for Health \& Social Protection SWAP.

Four new projects - Bishkek-Osh Infrastructure, Agricultural Investment and Services, Real Estate and Land Registration-II and Economic Management TA were approved by the Board FY08. There are currently three projects under preparation - Energy Emergency project, Osh- Isfana Road and Rural Water Sanitation-II. These projects are undergoing various stages of preparation process.

In addition to the four projects, the Board approved amendments in amount of 10 million USD to two ongoing projects Health \& Social Protection SWAP and AISP as a response to food price crisis. Both the Health SWAp portion ( $\$ 6$ million) and the agriculture support part (\$4.0 million) have been declared effective and actual disbursement for the Unified Monthly Benefit top-ups should commence with effect from October 1.

The Government has been highly appreciative of the World Bank's speedy response to the food price crisis and its targeted nature

In addition to the country portfolio, the Kyrgyz Republic also benefits from the regional HIV/AIDS project.

In overall, the World Bank own portfolio is in good shape with a disbursement ratio of $27.7 \%$ in FY08, and percent of projects at risk being a low 5.6

IFC Program and Portfolio: Since becoming a member of IFC in 1993, the

Kyrgyz Republic has received commitments totaling more than $\$ 70$ million from IFC's own funds to finance more then 20 projects in the financial, oil and gas, food and beverage, pulp and paper sectors. The committed IFC portfolio as of September 1, 2008 stands at \$20 million, with $\$ 13.4$ million disbursed (68\% in financial markets, $26 \%$ in general manufacturing and 6\% in agribusiness). In 2005-2008, IFC investments amounted to US\$30 million. In FY 2008, IFC approved and committed investments for Altyn-Ajydar for the production of construction materials, with a loan size of US\$1.6 million, and financing for Kyrgyz Investment Credit Bank (US\$4 million) to expand their activities. Currently, IFC is reviewing financing for a number of other projects in banking and finance sector. In addition, IFC intends to support the agribusiness sector by providing credit lines through local commercial banks for agribusiness companies. IFC is also considering possibilities of providing financing to two gold mining projects. In FY 2009 IFC plans to invest more than \$20 million dollars into the Kyrgyz economy.

IFC has completed and is implementing 17 technical assistance projects in the areas of (i) institutional and capacity building in the financial sectors including leasing, microfinance, (ii) creating favorable business environment for SMEs, (iii) improvement of investment climate, and (iv) developing capacity building for tourism. IFC PEP Central Asian Primary 
Market Development Project has been launched in 2005, and the second phase of IFC PEP Central Asian Regional Leasing Project was started. By the end of 2007, IFC plans to start regional TA project to improve corporate governance in enterprises of Central Asia including Kyrgyzstan.

MIGA: has supported private sector development in the Kyrgyz Republic by extending guarantees to foreign direct investments in four projects in the manufacturing, services, and mining sectors. MIGA's current portfolio in the Kyrgyz Republic consists of two projects, financed by Austrian and Italian investors, in support of the country's services sector. The combined gross exposure from these projects is US\$14.8 million.

Two claims have been filed relating to projects guaranteed by MIGA. One of them is the Kyrgyz Airlines project in which the Government of the Kyrgyz Republic cancelled the airline's license to operate, alleging that the investor breached material obligations under the license agreement. The case is in arbitration in London. The second dispute relates to the Manas Management Company project which handles the catering and cargo operations of the Manas International Airport in Bishkek. MIGA is seeking to settle the dispute through mediation, and is in close contact with both the Kyrgyz authorities and the investors for this purpose. The total amount of foreign direct investment facilitated by MIGA guarantees is over $\$ 360$ million. At present, there are no projects involving the Kyrgyz Republic in MIGA's FY08 pipeline.

World Bank contacts:

Roland Clarke (rclarke@worldbank.org), Sr. Country Economist. Phone No. (7-7272) 980580

Dinara Djoldosheva (ddjoldosheva@worldbank.org), Sr. Country Officer. Phone No. (996312) 610650 


\section{ANNEX III. KYRGYZ REPUBLIC-RELATIONS WITH THE ASIAN DEVELOPMENT BANK (ADB)}

(As of September 15, 2008)

ADB has been the second largest development partner in the Kyrgyz Republic since its joining in 1994. The current Joint Country Support Strategy (JCSS) for the period 2007-10 was prepared by ADB together with the United Kingdom's Department for International Development, Swiss Cooperation, United Nations agencies, and the World Bank Group ${ }^{4}$. The priority areas for JCSS are identified as its main pillars: (i) promoting private sector and economic growth, (ii) good governance and reducing corruption, and (iii) improving health, education and other social services. In line with the Government's own 2007-10 Country Development Strategy, ADB's future program will focus on (i) promoting economic management consistent with strong and sustained pro-poor growth; (ii) improving governance; promoting effective public administration and reducing corruption; (iii) building sustainable human and social capital; and (iv) ensuring environmental sustainability and natural resources management. It will be premised on ensuring environmental sustainability, private sector development, and regional cooperation.

The new country operations business plan (COBP) 2008-2011 is currently under preparation. While consistent with JCSS, the COBP entails some adjustments to ADB assistance program in the next few years. Two recent developments have necessitated making these adjustments. In January 2008, President Bakiev announced the New Economic Policy (NEP), calling upon the nation to refocus on economic growth and development after years of political uncertainty and languishing economic growth. In May 2008 ADB adopted Strategy 2020, which requires ADB to be more effective through more focused operations in five core areas. The adjustments made in the forward program are compliant with this approach.

As of 15 September 2008, the country has received 26 loans worth $\$ 603.5$ million and 5 ADF grants worth $\$ 66.1$ million. Seven out of 26 loans are program loans totaling \$199.5 million provided to support policy reforms to facilitate the transition to a market economy. The remaining 19 project loans and grants (worth $\$ 470.1$ million) were provided to support various investment activities. At present, 7 loans and 5 grants with approved amount of \$219.4 million are ongoing. These loans and grants have an un-disbursed balance of $\$ 124.7$ million as of 15 September, 2008. All assistance provided to the Kyrgyz Republic is from concessional ADB's special fund resources - Asian Development Fund (ADF). The Kyrgyz Republic has also received 7 grants from Japan Fund for Poverty Reduction (JFPR grants) amounting to $\$ 7$ million. The transport and communications sectors account for the largest share of ADB assistance, followed by the multisector, and law, economics and public policy.

\footnotetext{
${ }^{4}$ Two other development partners, namely the European Commission and the German Government joined the JCSS in December 2007.
} 
In addition to loans and grants, ADB had provided 70 technical assistance (TA) projects amounting to $\$ 40.351$ million as of today. Of these, 21 are project preparatory TAs amounting to $\$ 13.35$ million and the remaining 49 TAs for $\$ 27$ million are advisory TAs for capacity building, policy advice, institutional strengthening and training.

The performance of ADB's portfolio is generally satisfactory with no loan rated at risk. The scarcity of budgetary resources, and ceilings on the externally funded PIP constituted the biggest risks to the country portfolio. ADB and the World Bank have thus sought the removal of quarterly disbursement ceilings, which delay project implementation. In August 2005, IMF agreed to be more flexible in determining annual targets for the PIP, which is expected to improve portfolio performance.

ADB's annual lending began with $\$ 40$ million in 1994 and reached the peak level of $\$ 89$ million in 1997. Thereafter, lending declined as the Government's debt reduction strategy limited the size of its externally funded PIP to about 3 percent of GDP.

Since 2005, up to 50 percent of ADF assistance to the country was provided in the form of grants in view of the heavy external indebtedness of the country. In September 2007, the new grants framework was approved by ADB's Board of Directors, which enables the Kyrgyz Republic to receive 100 percent of its annual ADF allocation in grants. Access to grants will depend on the county's debt repayment capacity, which will need to be assessed periodically. As debt sustainability of the country strengthens, the Kyrgyz Republic will again be eligible for 50 percent grant and 50 percent loan starting from 2009. The allocation for the block of two years $2009-10$ is determined at $\$ 120.54$ million.

The Kyrgyz Republic was selected as one of the pilot countries during the February 2003 Rome Conference on Harmonization. Since then key development partners have learned to better coordinate and harmonize procurement procedures, oversee financial management and monitoring, share project implementation units, and conduct joint country portfolio reviews. The JCSS for the Kyrgyz Republic has been prepared by five funding agencies. Kyrgyz Resident Mission participated actively in the harmonization working group and contributed to the development of the National Action Plan for Harmonization which was approved by the Government in February 2005. The areas identified for harmonization in the immediate future are: (a) procedures for procurement of goods and services; (b) financial management and monitoring of projects; and (c) project implementation units. World Bank and ADB procurement documentation has been harmonized in these areas.

ADB cooperates extensively with civil society organizations in the Kyrgyz Republic to strengthen the effectiveness, quality, and sustainability of the services it provides. 


\section{ANNEX IV. KYRGYZ REPUBLIC-RELATIONS WITH THE EUROPEAN BANK FOR RECONSTRUCTION AND DEVELOPMENT (EBRD) (As of October 1, 2008)}

The EBRD facilitates the transition to a market-based economy through its direct support for private sector investment and key infrastructure, and targeted technical assistance. Under the Early Transition Countries' Initiative (ETCI) introduced in 2005, the Bank is able to more innovatively respond to the Kyrgyz economic requirements. Therefore, the Bank is considering smaller, "more challenging" projects. Under the ETCI, the Bank also provides technical cooperation (TC) in support of investment development.

In accordance with the Strategy for the Kyrgyz Republic approved in June 2007, the Bank's priorities are: (a) fostering the private sector; (b) strengthening the financial sector; (c) support for critical infrastructure; and (d) policy dialogue to improve the investment climate and support reform efforts.

As of October 2008, the Bank had approved 64 investment projects (including restructurings) with total financing of $€ 776$ million. The Bank has provided more than $€ 209.1$ million of this amount.

During the past three years, the Bank expanded its activities in the financial sector including the following:

- With an objective to develop dynamic micro, small and medium-sized enterprises, the Bank has been trying to strengthen the financial sector by investing and lending to stronger local financial institutions and encouraging coinvestment.

- The Bank works with seven partner banks and also with four nonbanking institutions-Bai-Tushum, FINCA, Kompanion and Frontiers.

- In 2007, the Bank has signed \$4 million with three nonbank’ institutions Frontiers, Bai-Tushum, and Kompanion. And

- In 2007, the Bank has approved \$40 million in support of the Bank’s Financial Sector Strategy for the Kyrgyz Republic which aims to strengthen the sector by addressing identified gaps and weaknesses and promoting best practice. The project will support development of the private enterprises and banking sector in the Kyrgyz Republic by increasing medium- and long-term financing to the productive sector of the economy through local banks. In 2007, under this project KICB received $\$ 3$ million credit line for lending to small- and medium-sized enterprises and \$2 million loan for mortgage financing. The SME credit line is used to finance local enterprises in the tourism sector and related services. The mortgage loan is used for financing residential mortgages.

- Equity investments in DKIB and KICB. 
- Expansion of Trade Facilitation Program (TFP). Four banks are participants in the TFP.

Other major investments by the Bank during its operations in KR include:

- $\$ 20$ million loan and \$10 million sub debt, since converted into \$17 million equity participation in Centerra Gold (the Bank's senior loan have been fully repaid).

- $\quad$ Loan to Hyatt-Regency Hotel, \$6.3 million in 1997.

- Loan to Interglass plant, \$6 million in 2004.

- Loan to Limatex (cotton-processing plant in Djalal-Abad), \$1 million in 2005.

- Loan to Karven Four Seasons (resort on Issyk-Kul Lake), \$3.8 million in 2006.

- Loan to Orion Hotel (hotel and business centre in Bishkek), \$4 million in 2007.

- Loan to Dairy Spring $€ 1.3$ in 2008.

The Bank provided the Kyrgyz Republic with sovereign guaranteed loans in the past and continues to monitor the development of key infrastructure projects in the public sector. The Bank is currently working with the Government for the possible EBRD financing of selected transport and municipal environmental infrastructure projects to be blended with concessional financing from other IFIs and bilateral donors. The Bank is also considering the possible non sovereign financing for airports, telecoms, gas pipeline projects to be financed on a commercial basis.

The Bank also implements grant-funded TC in support of investment projects, including the following ones in the FI (including MSFF consultants among others), natural resources/environment, agribusiness and infrastructure sectors:

- In telecoms, to advise on key reforms, including intercapacity access arrangements;

- Training for judges in commercial law;

- Business Advisory Service and Turn Around Management programs, providing consulting services to viable businesses.

The Bank actively promotes policy dialogue and implements selected TC activities to improve the business climate. The Bank supported the creation of an Investment Council to ensure high-level policy dialogue between the government and business community. ETC Fund resources ensures professional management of the Council, and the Bank participates in the process together with domestic and foreign private investors, as well as other donors. 


\section{Annex V. Kyrgyz Republic-Technical Assistance Provided by the Fund February 2003-November 2008}

\section{Dept. Subject/Identified Need}

FAD Improving the Effectiveness of the Large Taxpayer Unit

Treasury Management Information System

VAT on agriculture

Priorities for Tax Administration Reform

Supporting Tax Administration Reform and installing new Expert Advisor

Fiscal ROSC Reassessment (Fiscal Transparency Module)

MFD/ Review of the Capital Adequacy and MCM Dividend Arrangements for the National Bank of the Kyrgyz Republic

Review of Debt Restructuring Operation and 2003 Financial Reporting

Monetary Operations, Banking System Development, and Central Bank Autonomy

Review of NBKR Debt Restructuring Arrangements, Options for Deepening Financial Markets and Amendments to the NBKR Law

\section{Timing}

February 24-

March 7, 2003

July 21-29, 2003

November 3-11, 2003

July 22-August 5, 2004

January 16-28, 2006

September 11-25, 2007

August 18-28, 2003

October 28-November 10, 2003

September 13-23, 2004

December 7-18, 2004

\section{Counterpart}

Ministry of Finance

Ministry of Finance

Ministry of Finance

Ministry of Finance

Prime Minister's office

Ministry of Finance

National Bank of the Kyrgyz

Republic

National Bank of the Kyrgyz

Republic

National Bank of the Kyrgyz

Republic

National Bank of the

Kyrgyz Republic 


\section{Annex V. Kyrgyz Republic-Technical Assistance Provided by the Fund (Continued) February 2003-November 2008}

\begin{tabular}{|c|c|c|c|}
\hline Dept. & Subject/Identified Need & Timing & Counterpart \\
\hline & Payments System & $\begin{array}{l}\text { January } 25-\text { February } 7,2005, \\
\text { April 12-25, 2005, } \\
\text { October 18-27, 2005, } \\
\text { February 20-March 5, 2006, } \\
\text { October 16-27, 2006, March 3-17, } \\
\text { 2007, December 9-15, } 2007 \\
\text { May } 19 \text {-June 3, 2008 }\end{array}$ & $\begin{array}{l}\text { National Bank of } \\
\text { the Kyrgyz } \\
\text { Republic }\end{array}$ \\
\hline & Bank Supervision and Regulation & $\begin{array}{l}\text { February } 23-\text { March } 8,2005 \text {, May } \\
18-28,2005 \text {, July } 17-28,2005, \\
\text { October } 02-13,2005, \text { January } 15- \\
26,2006 \text {, February } 12-23,2006 \text {, } \\
\text { March } 20-30,2006\end{array}$ & $\begin{array}{l}\text { National Bank of } \\
\text { the Kyrgyz } \\
\text { Republic }\end{array}$ \\
\hline & FSAP update & $\begin{array}{l}\text { September } 5-15,2005 \\
\text { October } 10-23,2006\end{array}$ & $\begin{array}{l}\text { National Bank of } \\
\text { the Kyrgyz } \\
\text { Republic }\end{array}$ \\
\hline & $\mathrm{AML} / \mathrm{CFT}$ & April 19-25, 2006 & $\begin{array}{l}\text { National Bank of } \\
\text { the Kyrgyz } \\
\text { Republic }\end{array}$ \\
\hline & Islamic Banking Framework & February 4-12, 2008 & $\begin{array}{l}\text { National Bank of } \\
\text { the Kyrgyz } \\
\text { Republic }\end{array}$ \\
\hline \multirow[t]{4}{*}{ LEG } & $\begin{array}{l}\text { Update of the AML/CFT Legislation } \\
\text { (jointly with MFD) }\end{array}$ & February 5-11, 2004 & $\begin{array}{l}\text { National Bank of } \\
\text { the Kyrgyz } \\
\text { Republic }\end{array}$ \\
\hline & Review of Bank Legislation & $\begin{array}{l}\text { March 1-4, } 2004 \\
\text { April 26-May 6, } 2004\end{array}$ & $\begin{array}{l}\text { National Bank of } \\
\text { the Kyrgyz } \\
\text { Republic }\end{array}$ \\
\hline & Review of Tax Legislation & July 27-August 5, 2004 & Ministry of Finance \\
\hline & Assisting in drafting Tax Code & December 4-10, 2005 & $\begin{array}{l}\text { Prime Minister's } \\
\text { office }\end{array}$ \\
\hline
\end{tabular}


Annex V. Kyrgyz Republic-Technical Assistance Provided by the Fund (Concluded) February 2003-November 2008

\begin{tabular}{|c|c|c|c|}
\hline Dept. & Subject/Identified Need & Timing & Counterpart \\
\hline & AML/CFT Follow-up & July 2-6, 2007 & $\begin{array}{l}\text { National Bank of the } \\
\text { Kyrgyz Republic }\end{array}$ \\
\hline & AML/CFT Follow-up & September 3-6, 2007 & $\begin{array}{l}\text { National Bank of the } \\
\text { Kyrgyz Republic and } \\
\text { Financial Intelligence } \\
\text { Unit }\end{array}$ \\
\hline & AML/CFT Framework & $\begin{array}{l}\text { February } 11-15,2008 \\
\text { March 3-7, } 2008\end{array}$ & $\begin{array}{l}\text { National Bank of the } \\
\text { Kyrgyz Republic and } \\
\text { Financial Intelligence } \\
\text { Unit }\end{array}$ \\
\hline & Review of the draft Tax Code & April 22-30, 2008 & Ministry of Finance \\
\hline \multirow[t]{5}{*}{ STA } & SDDS Subscription & January 28-February 5, 2004 & $\begin{array}{l}\text { National Statistical } \\
\text { Committee }\end{array}$ \\
\hline & Balance of Payments Statistics & March 15-29, 2004 & $\begin{array}{l}\text { National Bank of the } \\
\text { Kyrgyz Republic }\end{array}$ \\
\hline & Monetary and Financial Statistics & April 27-May 11, 2004 & $\begin{array}{l}\text { National Bank of the } \\
\text { Kyrgyz Republic }\end{array}$ \\
\hline & National Accounts Statistics & November 17-28, 2008 & $\begin{array}{l}\text { National Statistics } \\
\text { Committee }\end{array}$ \\
\hline & Government Finance Statistics & November 11-14, 2008 & Ministry of Finance \\
\hline INS & Course in Financial Programming & November 12-20, 2008 & $\begin{array}{l}\text { Ministry of Economic } \\
\text { Development and } \\
\text { Trade \& others }\end{array}$ \\
\hline
\end{tabular}




\section{List of Resident Advisors}

\begin{tabular}{clll}
\hline MFD & $\begin{array}{l}\text { Banking Supervision/Restructuring } \\
\text { Advisor }\end{array}$ & Mr. Svartsman & $\begin{array}{l}\text { January 2004- } \\
\text { January 2005 }\end{array}$ \\
MFD & $\begin{array}{l}\text { Public Debt Policy and Management } \\
\text { MCM }\end{array}$ & $\begin{array}{l}\text { Mr. Azarbayejani } \\
\text { Debt Management and Development } \\
\text { Management }\end{array}$ & $\begin{array}{l}\text { December 2002- } \\
\text { December 2004 }\end{array}$ \\
& Mr. Riecke & August 2006- \\
August 2009
\end{tabular}




\section{ANNEX VI. KYRGYZ REPUBLIC-STATISTICAL ISSUES}

1. Data provision is adequate for surveillance. The four institutions responsible for collecting, compiling and disseminating macroeconomic statistics - the National Statistics Committee (NSC), the Ministry of Economic Development and Trade (MOEDT), the Ministry of Finance (MOF), and the National Bank of the Kyrgyz Republic (NBKR) - have legal and institutional environments that support statistical quality, and their respective staff are wellversed in current methodologies. Unlike staff resources, however, computer and financial resources are generally not commensurate with current needs and therefore constrain statistical development, especially for the NSC.

2. The NSC maintains a comprehensive and regularly updated website with data that largely incorporate international methodological recommendations with adequate coverage and timeliness (http://www.stat.kg). In February 2004, following improvements in compilation and dissemination of the reserves template and external debt data, the Kyrgyz Republic subscribed to the SDDS.

3. A data ROSC mission in November 2002 concluded that the quality of the macroeconomic statistics had improved significantly in the last few years. The authorities had established a good track record of implementing recommendations of past technical assistance and had demonstrated commitment to pursue plans and programs to further improve their statistics. The mission recommended that a program of regular intersectoral consistency checks be introduced to reduce the sometimes significant unexplained discrepancies between the government finance, monetary, and balance of payments datasets. The authorities' response to the data ROSC (posted on the IMF website (www.imf.org/external $/ \mathrm{np} / \mathrm{rosc}$ ) includes an update on the status of implementation of the ROSC recommendations.

\section{National accounts}

4. In general, dissemination of national accounts statistics is timely. Technical assistance has been received from the IMF, EUROSTAT, OECD, the World Bank, and bilateral donors. While significant progress has been made in improving the national accounts estimation process, problems persist regarding the quality of the source data, due mainly to excessively tight collection deadlines associated with the release schedule. Efforts are needed to improve the quality of the source data for quarterly GDP estimates. Moreover, while the quarterly GDP estimates are disseminated on a discrete basis for SDDS purposes, these estimates are still derived from cumulative data. Difficulties also remain in properly estimating the degree of underreporting, especially in the private sector. To improve the coverage and reliability of primary data, work has been undertaken to introduce sampling procedures. Improved sampling procedures have been adopted for household surveys and new report forms have been introduced for the enterprise survey. The NSC has established a division of sample surveys, which would assist in improving the sampling techniques. 


\section{Price and labor market statistics}

5. The concepts and definitions used in the CPI, which has been published since January 1995, are broadly consistent with international standards. The price index covers all urban resident households of all sizes and income levels, but needs to cover rural households, which comprise the majority of the population.

6. The PPI, which has been published since October 1996, is compiled broadly in accordance with international standards, although its coverage needs to be improved. The coverage of the PPI was broadened in May 1997 and is expected to be further expanded in the coming years.

7. Progress has been made in computing unit value indices for imports and exports. Work continues with regard to computation of these indices using a standard index presentation and the development of an export price index. However, problems in customs administration have led to incomplete coverage of trade and the lack of an appropriate valuation system. Moreover, the data processed by customs have suffered due to the use of an outdated computer software system.

8. Problems exist in the compilation of the average wage, especially with respect to the valuation of payments in kind and the coverage of the private sector. Monthly and annual data are not comparable because of different coverage and classifications. These problems extend to employment data as well. The coverage of unemployment includes an estimate of unregistered unemployed.

\section{Government finance statistics}

9. The scope of central government statistics falls short of international standards because it excludes data for the Social Fund and the externally financed Public Investment Program (these data are published separately). Other limitations involve the exclusion of financial transactions with domestic banks and the discrepancies between the deficit and financing data. While revenue and expenditure data generally accord with the GFSM 1986, there are misclassifications in both categories (for example, some nontax revenues are classified as taxes, and certain expenditure items are misclassified in the budget and treasury accounts). Monthly GFS data are reported to STA for publication in the IFS; the latest data reported for publication in the GFS Yearbook were for 2006, and covered general government and its subsectors; the data were compiled using the GFSM 2001 analytical framework.

10. The provision of data on public external debt service has improved. Data on actual debt service, guaranteed debt service, outstanding debt and revised debt projections, are provided on a monthly basis. The quality (including timeliness) of external debt data is adequate. The External Debt Division of the MOF is now solely responsible for monitoring external debt, and has benefited from on-site training provided by a Swiss-financed long-term consultant and the computerization of its database. 


\section{Monetary and financial statistics (MFS)}

11. The 2002 data ROSC mission found that: (a) the residency criterion was not uniformly applied, as the currency denomination was used to classify some transactions with foreign and domestic units; (b) deposits with banks in liquidation were included in broad money; and (c) source data did not provide sufficient information for a more detailed sectoral breakdown (e.g., subsectorization of nonbank institutions as recommended in the MFSM.

12. The April/May 2004 STA mission on MFS found that the NBKR had made substantial progress in implementing ROSC recommendations pertaining to monetary statistics. To address the outstanding issues, the mission further recommended that the NBKR (a) improve the basic source data to allow for proper classification of the transactions with foreign and domestic units; (b) fully implement the MFSM's methodology concerning accrual accounting; (c) exclude deposits with banks in liquidation from monetary aggregates and classify them as restricted deposits; and (d) set up a working group to follow up on consistency between monetary and balance of payments statistics. The mission also recommended expanding the current broad money survey to include the accounts of credit unions and microfinance companies.

13. Monetary data have been reported electronically to STA using Standardized Report Forms (SRFs). STA identified classification issues in the reported SRF data, which were communicated to the authorities. The data will be published in IFS and IFS Monetary and Financial Statistics Supplement as soon as these issues are resolved.

\section{External sector statistics}

14. Data on the balance of payments and international investment position are compiled and disseminated on a quarterly basis. The 2002 data ROSC mission noted that the compilation of balance of payments statistics broadly follows the methodology recommended in the BPM5. The NBKR has good arrangements with other agencies to ensure timely data flow. However, because of legal issues related to secrecy provisions, high value transactions cannot be verified with respondents, limiting the ability to cross-check the accuracy of data. Although the data collection program has been expanded in the recent past, coverage deficiencies remain with respect to trade, services, and foreign direct investment. The NBKR enterprise surveys lack an up-to-date register and have inadequate coverage of enterprises, particularly those in free economic zones. There is also a need to improve compilation procedures for achieving temporal consistency of data, and investigating and reconciling discrepancies.

15. The NSC conducts a quarterly sample survey for the estimation of shuttle trade, and uses customs records on the number of people crossing the border with CIS countries to derive the sample. The State Customs Inspectorate has introduced the customs receipt order for shuttle traders that simplifies and improves recording of imports of goods by shuttle traders. However, the high value limits applied for free import of goods by individuals have fostered a large shuttle trade, which has complicated estimation of this activity. The March 2004 STA mission on balance of payments statistics noted that while progress had been made in several areas, further improvements were needed in the international transactions reporting system; data sampling 
methods; and data validation and coverage, particularly on trade, services, private sector external debt, and foreign direct investment. The mission developed a questionnaire for collecting data on foreign direct investments and provided guidelines on the collection of data on external debt. 


\section{Kyrgyz Republic: Table of Common Indicators Required for Surveillance (As of November 17, 2008)}

\begin{tabular}{|c|c|c|c|c|c|c|c|}
\hline & $\begin{array}{c}\text { Date of latest } \\
\text { observation }\end{array}$ & Date received & Frequency & Frequency & $\begin{array}{c}\text { Frequency } \\
\text { of }\end{array}$ & \multicolumn{2}{|c|}{ Memo Items: } \\
\hline & & & & & & $\begin{array}{l}\text { Data Quality- } \\
\text { Methodologica } \\
\text { I soundness }^{8}\end{array}$ & $\begin{array}{l}\text { Data Quality- } \\
\text { Accuracy and } \\
\text { reliability }^{9}\end{array}$ \\
\hline Exchange Rates & $11 / 17 / 08$ & $11 / 17 / 08$ & $\mathrm{D}$ & $\mathrm{D}$ & W & & \\
\hline $\begin{array}{l}\text { International Reserve Assets and Reserve } \\
\text { Liabilities of the Monetary Authorities }^{1}\end{array}$ & $10 / 31 / 08$ & $11 / 17 / 08$ & M & M & M & & \\
\hline Reserve/Base Money & $11 / 14 / 08$ & $11 / 17 / 08$ & $\mathrm{D}$ & $\mathrm{D}$ & M & LO, O, LO, LO & $\mathrm{LO}, \mathrm{O}, \mathrm{O}, \mathrm{LO}, \mathrm{LO}$ \\
\hline Broad Money & $9 / 30 / 08$ & $10 / 16 / 08$ & M & M & M & & \\
\hline Central Bank Balance Sheet & $11 / 14 / 08$ & $11 / 17 / 08$ & $\mathrm{D}$ & $\mathrm{D}$ & M & & \\
\hline $\begin{array}{l}\text { Consolidated Balance Sheet of the Banking } \\
\text { System }\end{array}$ & $9 / 30 / 08$ & $10 / 16 / 08$ & M & M & M & & \\
\hline Interest Rates $^{2}$ & $11 / 13 / 08$ & $11 / 17 / 08$ & W & W & W & & \\
\hline Consumer Price Index & $10 / 30 / 08$ & $11 / 14 / 08$ & M & M & M & $\mathrm{O}, \mathrm{LO}, \mathrm{O}, \mathrm{O}$ & LO, LO, O, O, O \\
\hline $\begin{array}{l}\text { Revenue, Expenditure, Balance and } \\
\text { Composition of Financing }^{3} \text {-General } \\
\text { Government }^{4}\end{array}$ & $9 / 30 / 08$ & $10 / 25 / 08$ & $\mathrm{M}$ & M & A & O, LNO, LO, O & LO, O, O, LO, LO \\
\hline $\begin{array}{l}\text { Revenue, Expenditure, Balance and } \\
\text { Composition of Financing }{ }^{3} \text {-Central } \\
\text { Government }\end{array}$ & $9 / 30 / 08$ & $10 / 25 / 08$ & M & M & M & & \\
\hline $\begin{array}{l}\text { Stocks of Central Government and Central } \\
\text { Government-Guaranteed Debt }{ }^{5}\end{array}$ & $12 / 31 / 07$ & $6 / 30 / 08$ & M & M & A & & \\
\hline External Current Account Balance & $6 / 30 / 08$ & $10 / 10 / 08$ & Q & Q & Q & LO, LO, LO, LO & $\begin{array}{l}\text { O, LO, LO, LO, } \\
\text { LO }\end{array}$ \\
\hline Exports and Imports of Goods and Services & $6 / 30 / 08$ & $10 / 10 / 08$ & Q & Q & Q & & \\
\hline GDP/GNP & $10 / 31 / 08$ & $11 / 12 / 08$ & M & M & M & $\mathrm{O}, \mathrm{O}, \mathrm{LO}, \mathrm{O}$ & LO, LO, LO, O, O \\
\hline Gross External Debt & $12 / 31 / 07$ & $6 / 30 / 08$ & Q & Q & A & & \\
\hline International Investment Position ${ }^{6}$ & 2007 & $6 / 11 / 08$ & & & & & \\
\hline
\end{tabular}

${ }^{1}$ Includes reserve assets pledged or otherwise encumbered, as well as net derivative positions.

${ }^{2}$ Both market-based and officially determined, including discount rates, money market rates, rates on treasury bills, notes and bonds.

${ }^{3}$ Foreign and domestic financing only.

${ }^{4}$ The general government consists of the central government (budgetary funds, extra budgetary funds, and social security funds) and state and local governments.

${ }^{5}$ Including currency and maturity composition.

${ }^{6}$ Includes external gross financial asset and liability positions vis-à-vis nonresidents.

${ }^{7}$ Daily (D), Weekly (W), Monthly (M), Quarterly (Q), Annually (A), Irregular (I); Not Available (NA).

${ }^{8}$ Reflects the assessment provided in the data ROSC (published in November 2003, and based on the findings of the mission that took place during November 2002) for the dataset corresponding to the variable in each row. The assessment indicates whether international standards concerning concepts and definitions, scope, classification/sectorization, and basis for recording are fully observed (O), largely observed (LO), largely not observed (LNO), or not observed (NO).

${ }^{9}$ Same as footnote 7, except referring to international standards concerning source data, statistical techniques, assessment and validation of source data, assessment and validation of intermediate data and statistical outputs, and revision studies 


\title{
INTERNATIONAL MONETARY FUND
}

\author{
KYRGYZ REPUBLIC
}

\section{Joint World Bank/IMF Debt Sustainability Analysis \\ Prepared by the Staffs of the International Monetary Fund and the World Bank}

Approved by David Owen and Adnan Mazarei (IMF) and

Carlos A. Primo Braga and Luca Barbone (World Bank)

December 1, 2008

Based on the joint World Bank-IMF Low-Income Country Debt Sustainability Framework, the Kyrgyz Republic is assessed to be at a moderate risk of external debt distress. ${ }^{5}$ Compared to the previous joint DSA, the debt outlook looks more favorable. However, some stress tests suggest that the country is still vulnerable, particularly to exogenous shocks or a reversal in real GDP growth. Further improvement of the debt outlook will depend on maintaining sound macroeconomic policies; including prudent borrowing, as well as assuring continued concessional financing to support the country's large development needs.

\section{BACKGROUND}

\section{The Kyrgyz Republic's nominal stock of public and publicly guaranteed (PPG) external} debt declined from about 100 percent in 2003 to 70 percent of GDP in 2006, and further to 55 percent in 2007. The decline has been mainly the result of an acceleration in the pace of economic growth and a nominal appreciation of the domestic currency, but also reflects firm fiscal discipline and Paris Club support. The present value (PV) of PPG external debt was equivalent to $\$ 1,372$ million (36.6 percent of GDP) at end-2007, of which 72 percent is owed to international financial institutions (IFIs) and the remaining 28 percent to bilateral creditors. The external private debt reached almost 6 percent of GDP at end-2007.

\section{The Kyrgyz Republic has had two debt restructuring agreements with the Paris Club.} The first debt restructuring with the Paris Club, in December 2002, provided for flow rescheduling, in three phases and on Houston terms, of maturities falling due between December 2001 and April 2005, all of which have been implemented. The second, in March 2005, under the Evian approach, stipulated that the full amount of principal payments on official development assistance (ODA) credits was to be repaid over 40 years, with a

\footnotetext{
${ }^{5}$ The DSA has been produced jointly by Bank and Fund staffs, in consultation with Asian Development Bank staff and the Kyrgyz authorities. The fiscal year for the Kyrgyz Republic is January 1-December 31.
} 
13-year grace period, at interest rates at least as favorable as the original concessional rates applied to those loans. On non-ODA credit, creditors agreed to halve the NPV, following either debt reduction or debt service reduction options. The Kyrgyz authorities indicated in early 2007 that they did not wish to avail themselves of the HIPC initiative but subsequently expressed interest for the MDRI. At end-2007, indebtedness indicators were estimated to be below the applicable HIPC Initiative thresholds, while income levels were estimated to be above the IMF MDRI thresholds.

\section{UNDERLYING DEBT SUSTAINABILITY ANALYSIS ASSUMPTIONS}

The macroeconomic assumptions reflect the framework underlying the prospective IMF program and World Bank and IMF staff projections through 2028. They have been updated to incorporate recent developments and changes to the medium-term outlook, but long-term assumptions are broadly similar to the framework used in the last LIC DSA. The data on the stock of external debt at end-2007 were provided by the Kyrgyz authorities.

The framework assumes continuation of sound macroeconomic policies-including fiscal consolidation and prudent public debt management—as a basis for sustaining growth (Box 1). The baseline projections do not rely on substantially higher growth rates than the historical average nor on significant improvement in loan terms. Growth would be underpinned by firm implementation of structural reforms to remove impediments to private investment and stimulate economic diversification. The framework features average long-run GDP growth of about 5 percent per year over 2014-28, broadly in line with growth assumed in the last DSA, backed by strong private investments (including foreign direct investment) spurred by improvements in the business climate. The external current account deficit is projected to decline from 8.5 percent of GDP in 2009 to 3.4 percent in 2013, but to average 4.4 percent in 2014-28 following the gradual decline of gold output.

The last DSA underestimated the near-term GDP growth. In particular, actual 2007 GDP in U.S. dollar terms was 71/2 percent higher than the level projected in the last DSA. In addition, exports and the current account deficit in 2007 were also substantially lower than the levels projected earlier. 


\section{Box 1. Baseline Macroeconomic Assumptions}

Real GDP growth is projected to average about 5 percent over the longer term, only marginally higher than the historical average of about $4 \frac{1}{2}$ percent, on the back of strong private investment, including FDI, spurred by improvements in the business climate. Affected by the global and regional slowdown combined with weaker domestic demand due to high inflation and slowdown in credit expansion, GDP growth is expected to slow down in the near term. In the medium term, however, growth will rebound supported by a continued recovery in the mining industry and the resumed reforms in the energy sector, while tourism-related services and a reformed energy sector would underpin measured but sustained long-run growth. Consistent with the assumption of sound fiscal and monetary policies, inflation (measured by the GDP deflator) would average 41/2 percent annually over 2011-28.

Recovering from an accident in the Kumtor gold mine in 2006, gold output is expected to grow substantially in 2008-09. Long-run projections assume that a sharp drop in gold output from the expected closure of the existing Kumtor mine in 2014-15 will be compensated by gains in tourism receipts and other exports, as well as an increase in production in new gold mines. In all, annual growth of exports of goods and services, including estimates for informal agricultural exports and reexports of consumer goods, would average 6.3 percent over the long term, maintaining the exportto-GDP ratio at about 53 percent. Consistent with growth projections and expected FDI inflows, imports of goods and services would grow at about 6.6 percent per year over the long term, with the import-to-GDP ratio declining only slightly to 85 percent. Remittances are projected to slow down somewhat in the near term, but will remain strong over medium and long term, with their ratio to GDP slowly declining.

Net FDI inflows would stay at about $3 \frac{1}{2}$ percent of GDP over the medium and long term. While in the near-term FDI would be concentrated in traditional sectors, like mining and industry, business climate improvements should yield a more diversified structure of FDI in the outer years. International reserves would be kept above three months of imports of goods and services.

Medium-term public borrowing is assumed to be on highly concessional terms, primarily from IFIs. Over the DSA horizon, concessionality of new external public borrowing would gradually decline from around 41.8 percent in 2008-13 to 15.8 percent in 2014-28, as more borrowing will be contracted at less concessional terms from bilateral and commercial creditors.

A new Tax Code that aims at harmonizing the tax rates with those of the neighboring countries will become effective in 2009. VAT rates will go down from 20 percent to 12 percent, and the existing sales, road, and emergency taxes will be eliminated. A new turnover tax with a broader base and a property tax will be introduced that aim at offsetting losses from the tax cuts. Tighter control of goods and services spending, as well as improved tax administration will prevent the primary deficit from rising significantly. As the primary deficit is projected to be stable at about one percent of GDP in 2009-28, the public sector debt declines to about 45 percent of GDP by 2028, from about 60 percent in 2007, reflecting a prudent public debt management strategy of the government. After the closure of the currently operating Kumtor mine by 2014-15, the primary deficit will increase to about $1 \frac{1}{2}$ percent of GDP in 2014-15, but will return to one percent on average thereafter. 


\title{
III. EXTERnal Debt Sustainability Analysis
}

\begin{abstract}
A. Baseline
The baseline scenario points to a cautiously favorable improvement in the external debt outlook over time. Already by end-2007, the PV of debt-to-exports and the PV of debt-torevenue ratios, at 70.6 and 128.4 percent respectively, were well below their policy-based indicative thresholds. ${ }^{6}$ Only the PV of PPG debt-to-GDP ratio, at 37 percent, is close to its relevant threshold of 40 percent in 2007, but by end-2008 it is projected to fall to 29.3 percent. Over the DSA horizon, all debt ratios move steadily on a downward path underpinned by steady growth, fiscal consolidation, and prudent debt management. With the anticipated closure of the existing Kumtor gold mine operations in 2014-15, the ratios would increase somewhat around that time, but would eventually fall back below the pre-closure levels. The DSA exercise does not incorporate potential debt financing of the Kambar-Ata hydroelectric project as it reflects the authorities' current plan to develop the project on the basis of equity participation.
\end{abstract}

Debt service is expected to remain manageable throughout the DSA horizon. This reflects the high concessionality of both the outstanding multilateral debt and the assumed new borrowing, as well as the debt relief delivered by Paris Club creditors in 2005. The PPG debt service ratio would increase slightly from 2 percent of exports ( 4.7 percent of revenues) in 2013 to 3.9 percent of exports ( 7.9 percent of revenues) in 2028, driven by lessconcessional new borrowing and the repayment of the previously restructured bilateral debt.

\section{B. Alternative Scenarios and Stress Tests}

\section{Stress tests and alternative scenarios show that the Kyrgyz Republic's external debt is vulnerable to large shocks or substantially less favorable assumptions. The PV of debt- to-GDP ratio and the PV of debt-to-revenue rise above the relevant indicative thresholds under some tests. The PV of debt-to-GDP ratio rises above the indicative threshold of 40 percent in the medium term when the net non-debt creating inflows over 2009-2010 are one standard deviation below their historical average, or under a shock over 2009-2010 combining lower GDP and export growth, currency depreciation, and lower net nondebt creating inflows. The PV of debt-to-revenue would also rise above the relevant indicative threshold of 250 percent in the short term under these conditions. However, the PV of debt- to-exports ratio is robust and does not breach its threshold under various tests. Debt service ratios also prove resilient, staying below their indicative threshold levels under various tests.}

\footnotetext{
${ }^{6}$ The Kyrgyz Republic is rated as a medium performer based on the World Bank's Country Performance and Institutional Assessment Index for low income countries. The relevant policy-dependent thresholds for countries in this category are 40 percent for the PV of the debt-to-GDP ratio, 150 percent for the PV of debt-toexports ratio, 250 percent for the PV of debt-to-revenue ratio, 20 percent of the debt service-to-exports ratio, and 30 percent of the debt service-to-revenue ratio.
} 
The historical scenario - where key macro variables evolve according to their historic averages - points to a more benign external debt outlook than the baseline scenario.

\title{
IV. Public Debt Sustainability Analysis
}

\begin{abstract}
A. Baseline
Domestic debt is projected to increase and will play a more important role in financing the budget deficit in the medium and long term. Domestic debt currently accounts for less than 10 percent of total public debt. However, by 2028, domestic debt is projected to exceed one-third of total public debt as domestic financial markets deepen.
\end{abstract}

The Kyrgyz Republic public debt outlook is projected to be manageable in the medium and long term. Under the baseline scenario, the PV of public debt to GDP ratio goes down to $26 \frac{1}{2}$ percent in 2013 from about 40 percent in 2007, but increases to $41 \frac{1}{2}$ percent of GDP by 2028. The tax revenue ratio will increase from 23 percent in 2007 to $271 / 2$ percent in 2028 . The tax revenue ratio will drop somewhat in 2009 due to tax cuts envisaged in the new tax code. Also, in 2014 and 2015, the income tax ratio will drop as the existing Kumtor mine closes. However, in the longer run, income taxes will gradually recover and, aided by discipline on the expenditure side, contribute to the sustainability of public debt indicators.

\section{B. Alternative Scenarios and Stress Tests}

Alternative scenarios and stress tests show that Kyrgyz Republic's public debt remains sensitive to shocks that reduce real GDP growth. The standard sensitivity analysis based on the historical variation of key parameters, including real GDP growth and exchange rate, shows that debt ratios will rise considerably in the long run. Under different stress tests and scenarios, the PV debt-to-GDP ratio in 2028 will vary from about 35 percent under the fixed (at 2008 level) primary deficit scenario to 98 percent under the permanent real GDP growth shock scenario.

\section{DebT Distress Classification and Conclusions}

Based on the projected external debt burden indicators, Kyrgyz Republic is assessed to be at moderate risk of debt distress. The public DSA suggests that Kyrgyz Republic's total public sector debt seems manageable in light of the dynamics of the domestic debt stock. All NPV-based external debt indicators in the baseline are projected to stay below their indicative thresholds over the DSA horizon. Moreover, under the baseline scenario, the debt service burden would remain well below the thresholds, reflecting the high concessionality of the external debt. Nevertheless, alternative scenarios and stress tests show that the external public debt indicators could approach or breach the thresholds if the Kyrgyz Republic were 
to experience large adverse exogenous shocks or relax its prudent debt management policy. This conclusion is consistent with the last DSA.

Low-concessionality loans from bilateral and commercial creditors to finance large public investment projects continue to pose a risk to the debt outlook. Staff recognizes that the Kyrgyz Republic has large developmental needs, but considers paramount to lock in the recent progress towards achieving and maintaining debt sustainability. Even if loans have a grant element of at least 35 percent, it would be important to ensure that the underlying projects are viable and that market risks, including exchange rate risk, are accounted for, so as to avoid the build up of an unsustainable debt burden. 
Figure 1. Indicators of Public and Publicly Guaranteed External Debt under Alternatives Scenarios, 2008-28 1/

a. Debt Accumulation

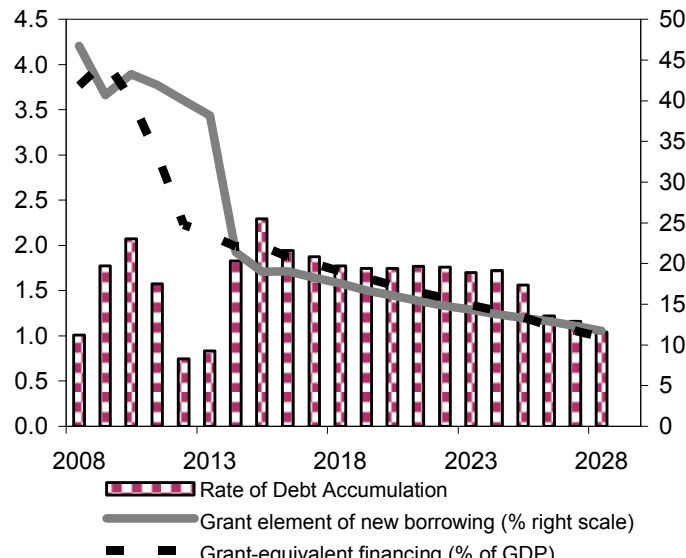

- Grant-equivalent financing (\% of GDP)
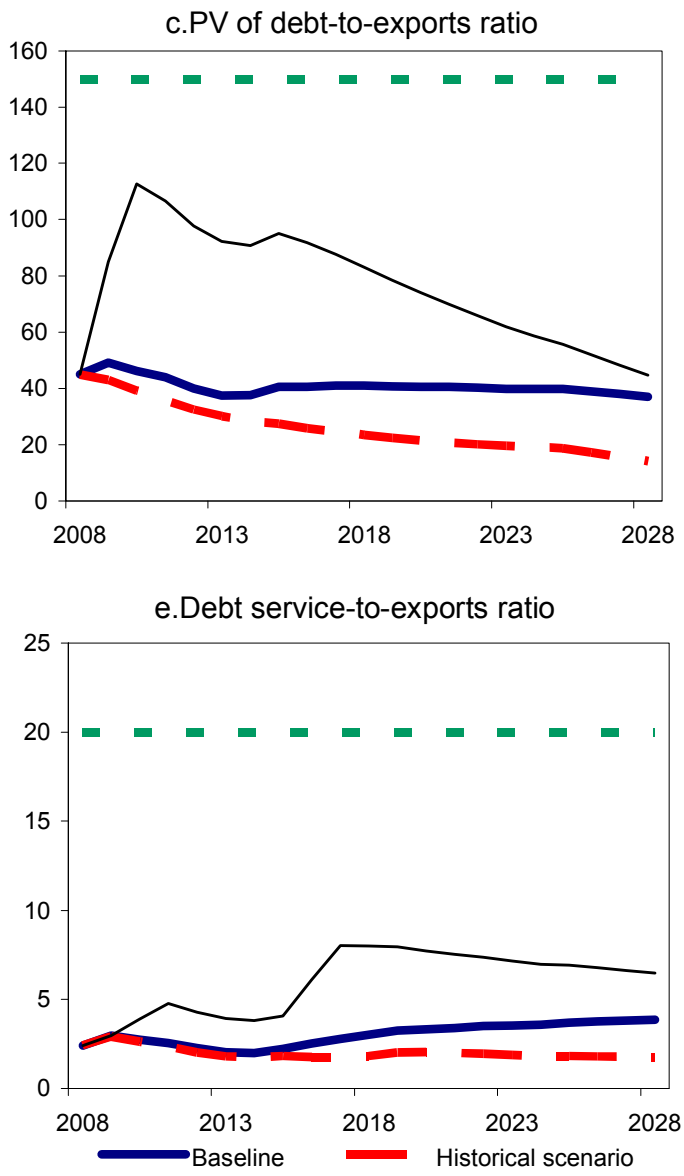

b.PV of debt-to GDP ratio
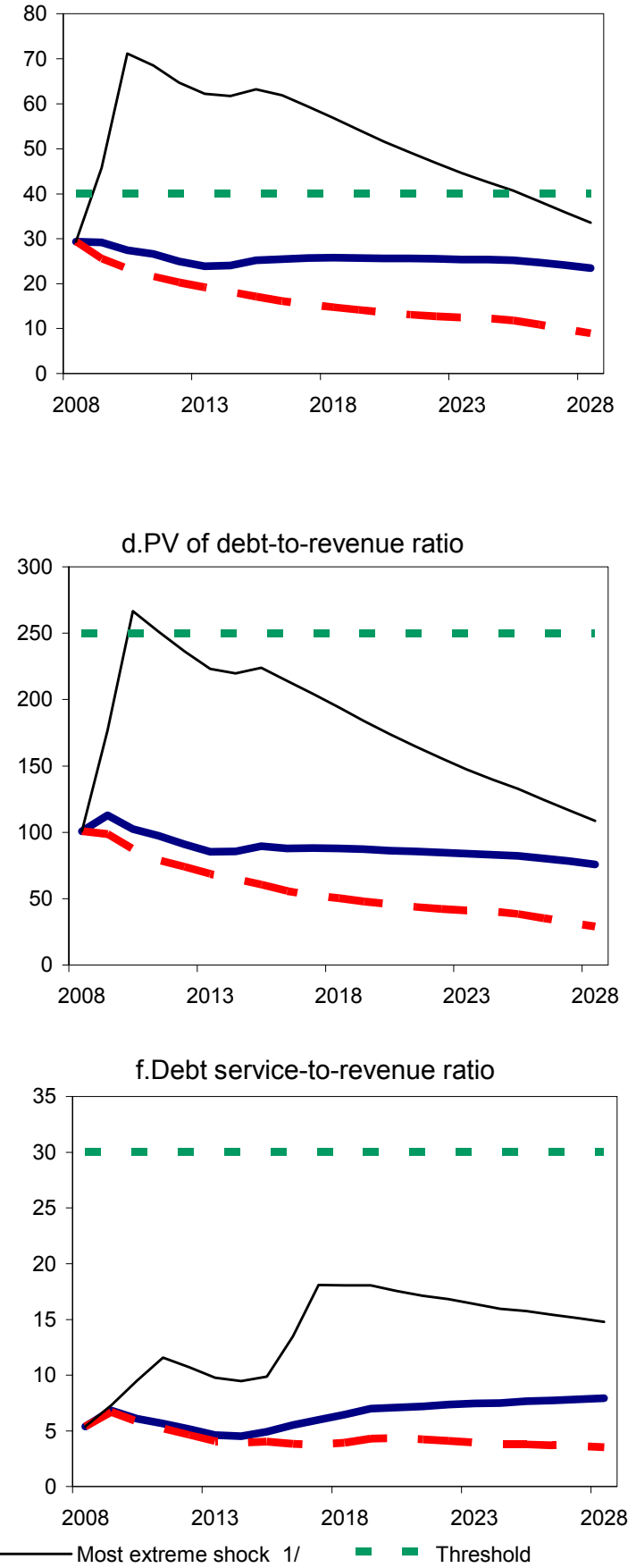

Source: Staff projections and simulations.

$1 /$ The most extreme stress test is the test that yields the highest ratio in 2018. In figure $\mathrm{b}$. it corresponds to a Combination shock; in c. to a Combination shock; in d. to a Combination shock; in e. to a Combination shock and in picture $f$. to a Combination shock 
Figure 2. Indicators of Public Debt Under Alternative Scenarios, 2008-28 1/

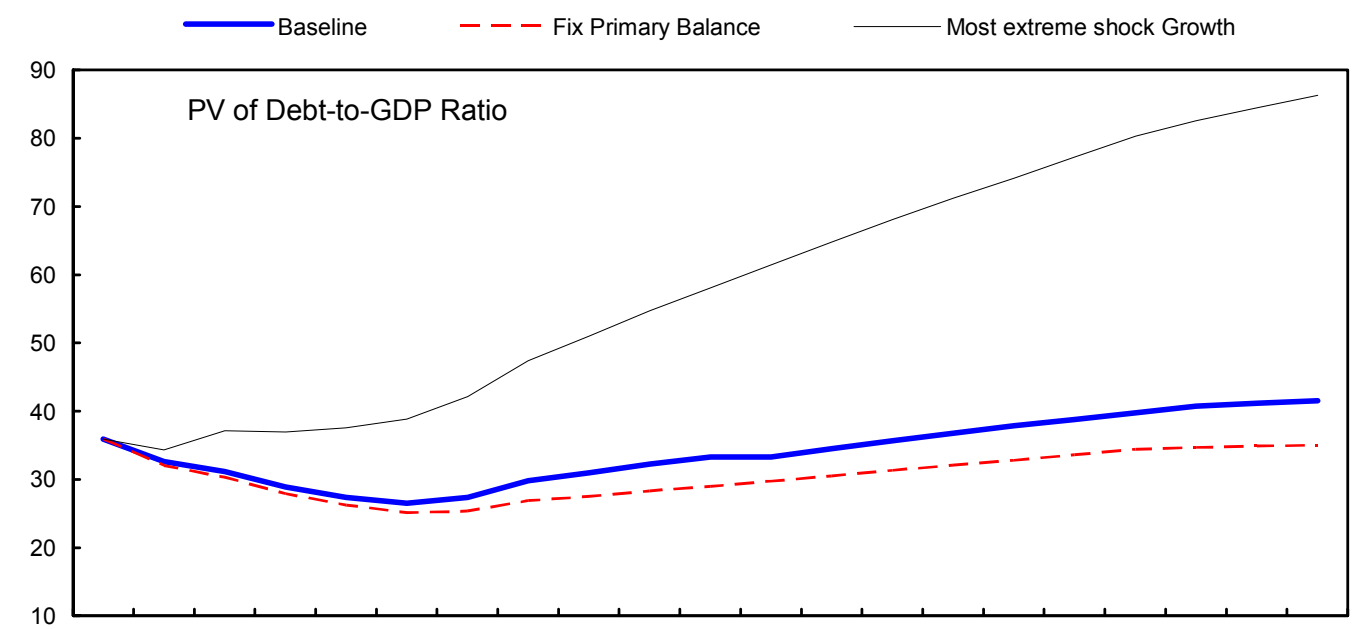

200820092010201120122013201420152016201720182019202020212022202320242025202620272028

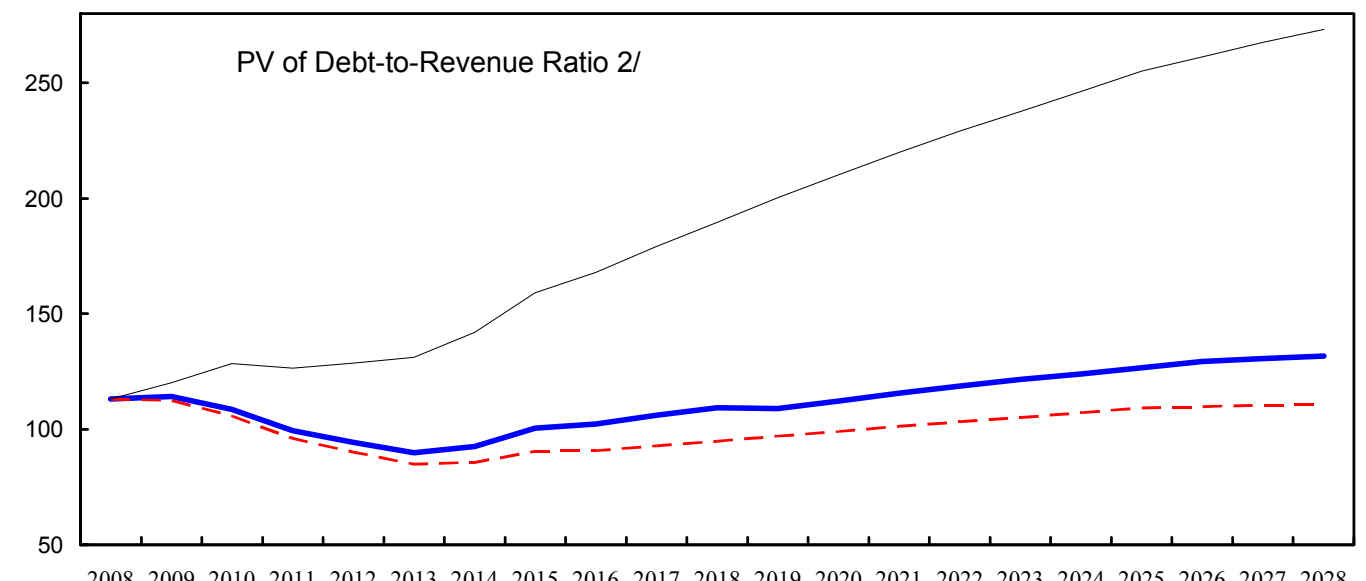

200820092010201120122013201420152016201720182019202020212022202320242025202620272028

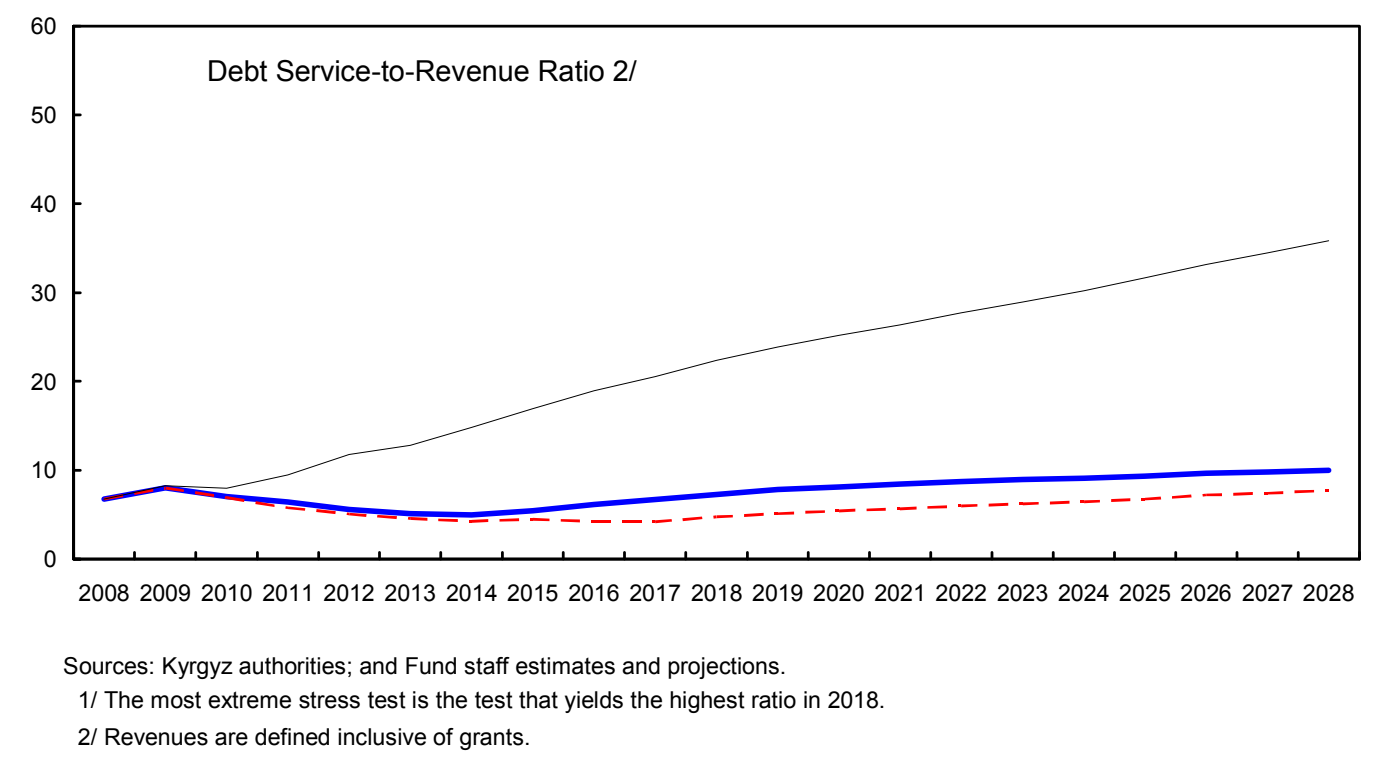


Table 1a. Public Sector Debt Sustainability Framework, Baseline Scenario, 2005-28 (In percent of GDP, unless otherwise indicated)

\begin{tabular}{|c|c|c|c|c|c|c|c|c|c|c|c|c|c|c|c|}
\hline & \multicolumn{3}{|c|}{ Actual } & \multirow[b]{2}{*}{ Average } & \multirow[b]{2}{*}{$\begin{array}{l}\text { Standard } \\
\text { Deviation }\end{array}$} & \multicolumn{4}{|l|}{ stimate } & \multicolumn{3}{|c|}{ Projections } & \multirow[b]{2}{*}{2018} & \multirow[b]{2}{*}{2028} & \multirow[b]{2}{*}{$\begin{array}{l}2014-28 \\
\text { Average }\end{array}$} \\
\hline & 2005 & 2006 & 2007 & & & 2008 & 2009 & 2010 & 2011 & 2012 & 2013 & $\begin{array}{c}2008-13 \\
\text { Average }\end{array}$ & & & \\
\hline $\begin{array}{l}\text { Public sector debt } 1 / \\
\text { o/w foreign-currency denominated }\end{array}$ & $\begin{array}{l}85.9 \\
80.4\end{array}$ & $\begin{array}{l}72.5 \\
67.9\end{array}$ & $\begin{array}{l}57.7 \\
53.7\end{array}$ & & & $\begin{array}{l}52.2 \\
49.0\end{array}$ & $\begin{array}{l}47.2 \\
44.6\end{array}$ & $\begin{array}{l}45.1 \\
42.5\end{array}$ & $\begin{array}{l}42.1 \\
40.9\end{array}$ & $\begin{array}{l}39.4 \\
37.9\end{array}$ & $\begin{array}{l}37.5 \\
35.7\end{array}$ & & $\begin{array}{l}41.1 \\
34.1\end{array}$ & $\begin{array}{l}45.2 \\
27.5\end{array}$ & \\
\hline $\begin{array}{l}\text { Change in public sector debt } \\
\text { Identified debt-creating flows }\end{array}$ & $\begin{array}{l}-7.0 \\
-2.0\end{array}$ & $\begin{array}{l}-13.4 \\
-12.8\end{array}$ & $\begin{array}{l}-14.8 \\
-16.8\end{array}$ & & & $\begin{array}{l}-5.5 \\
-4.9\end{array}$ & $\begin{array}{l}-5.0 \\
-5.0\end{array}$ & $\begin{array}{l}-2.1 \\
-3.6\end{array}$ & $\begin{array}{l}-2.9 \\
-2.5\end{array}$ & $\begin{array}{l}-2.7 \\
-2.5\end{array}$ & $\begin{array}{l}-1.9 \\
-1.6\end{array}$ & & $\begin{array}{r}0.5 \\
-0.9\end{array}$ & $\begin{array}{l}-0.2 \\
-1.2\end{array}$ & \\
\hline Primary deficit $2 /$ & 1.7 & 1.5 & 0.0 & 4.3 & 3.5 & 0.5 & 0.9 & 0.7 & 0.6 & 0.7 & 0.8 & 0.7 & 1.0 & 0.5 & 0.9 \\
\hline Revenue and grants & 24.7 & 26.4 & 30.8 & & & 31.7 & 28.5 & 28.7 & 29.0 & 29.0 & 29.5 & & 30.5 & 31.5 & \\
\hline of which: grants & 1.0 & 0.8 & 2.3 & & & 2.7 & 2.6 & 2.0 & 1.7 & 1.6 & 1.6 & & 1.2 & 0.7 & \\
\hline Primary (noninterest) expenditure & 26.4 & 27.9 & 30.8 & & & 32.3 & 29.4 & 29.4 & 29.6 & 29.8 & 30.2 & & 31.5 & 32.1 & \\
\hline Automatic debt dynamics & -3.5 & -14.3 & -16.7 & & & -5.6 & -5.8 & -4.3 & -3.0 & -3.1 & -2.3 & & -1.8 & -1.6 & \\
\hline Contribution from interest rate/growth differential & -1.2 & -4.3 & -6.7 & & & -4.6 & -1.8 & -2.6 & -2.2 & -2.9 & -2.3 & & -1.7 & -1.6 & \\
\hline of which: contribution from average real interest rate & -1.3 & -1.7 & -1.2 & & & -0.5 & 0.1 & 0.1 & -0.1 & -0.1 & -0.1 & & 0.2 & 0.6 & \\
\hline of which: contribution from real GDP growth & 0.1 & -2.6 & -5.5 & & & -4.0 & -1.9 & -2.7 & -2.1 & -2.8 & -2.2 & & -1.9 & -2.2 & \\
\hline Contribution from real exchange rate depreciation & -2.3 & -10.0 & -9.9 & & & -1.1 & -4.0 & -1.7 & -0.8 & -0.2 & 0.0 & & $\ldots$ & $\ldots$ & \\
\hline Other identified debt-creating flows & -0.2 & 0.0 & -0.1 & & & 0.1 & -0.1 & -0.1 & -0.1 & -0.1 & -0.1 & & -0.1 & 0.0 & \\
\hline Privatization receipts (negative) & -0.2 & 0.0 & -0.1 & & & 0.1 & -0.1 & -0.1 & -0.1 & -0.1 & -0.1 & & -0.1 & 0.0 & \\
\hline Recognition of implicit or contingent liabilities & 0.0 & 0.0 & 0.0 & & & 0.0 & 0.0 & 0.0 & 0.0 & 0.0 & 0.0 & & 0.0 & 0.0 & \\
\hline Debt relief (HIPC and other) & -0.1 & 0.0 & 0.0 & & & 0.0 & 0.0 & 0.0 & 0.0 & 0.0 & 0.0 & & 0.0 & 0.0 & \\
\hline Other (specify, e.g. bank recapitalization) & 0.0 & 0.0 & 0.0 & & & 0.0 & 0.0 & 0.0 & 0.0 & 0.0 & 0.0 & & 0.0 & 0.0 & \\
\hline Residual, including asset changes & -5.0 & -0.7 & 2.0 & & & -0.5 & 0.0 & 1.5 & -0.5 & -0.2 & -0.3 & & 1.3 & 1.0 & \\
\hline \multicolumn{16}{|l|}{ Other Sustainability Indicators } \\
\hline PV of public sector debt & 54.3 & 48.3 & 39.8 & & & 35.9 & 32.5 & 31.1 & 28.8 & 27.4 & 26.5 & & 33.4 & 41.5 & \\
\hline $\mathrm{o} / \mathrm{w}$ foreign-currency denominated & 48.8 & 43.6 & 35.9 & & & 32.7 & 30.0 & 28.5 & 27.6 & 25.9 & 24.8 & & 26.4 & 23.8 & \\
\hline o/w external & 46.9 & 42.0 & 34.6 & & & 31.7 & 29.1 & 27.8 & 26.9 & 25.3 & 24.2 & & 26.0 & 23.7 & \\
\hline PV of contingent liabilities (not included in public sector debt) & $\ldots$ & & $\ldots$ & & & $\ldots$ & & $\ldots$ & & $\ldots$ & & & & $\ldots$ & \\
\hline Gross financing need $3 /$ & 9.1 & 6.1 & 5.9 & & & 3.4 & 3.6 & 4.2 & 3.6 & 3.4 & 2.8 & & 4.6 & 8.9 & \\
\hline PV of public sector debt-to-revenue and grants ratio (in percent) & 219.9 & 182.7 & 129.3 & & & 113.0 & 114.1 & 108.5 & 99.3 & 94.5 & 90.0 & & 109.3 & 131.6 & \\
\hline PV of public sector debt-to-revenue ratio (in percent) & 229.4 & 188.3 & 139.8 & & & 123.3 & 125.7 & 116.5 & 105.7 & 100.1 & 95.0 & & 113.8 & 134.5 & \\
\hline o/w external $4 /$ & 198.0 & 163.9 & 121.5 & & & 108.9 & 112.6 & 104.0 & 98.7 & 92.4 & 86.8 & & 88.9 & 76.7 & \\
\hline Debt service-to-revenue and grants ratio (in percent) $5 /$ & 12.7 & 10.0 & 6.5 & & & 6.8 & 8.0 & 7.0 & 6.4 & 5.6 & 5.1 & & 7.3 & 10.0 & \\
\hline Debt service-to-revenue ratio (in percent) $5 /$ & 13.3 & 10.3 & 7.0 & & & 7.4 & 8.8 & 7.6 & 6.9 & 5.9 & 5.4 & & 7.6 & 10.2 & \\
\hline Primary deficit that stabilizes the debt-to-GDP ratio & 8.7 & 15.0 & 14.8 & & & 6.0 & 5.9 & 2.9 & 3.5 & 3.5 & 2.7 & & 0.5 & 0.7 & \\
\hline \multicolumn{16}{|l|}{ Key macroeconomic and fiscal assumptions } \\
\hline Real GDP growth (in percent) & -0.2 & 3.1 & 8.2 & 4.4 & 4.1 & 7.5 & 3.7 & 6.2 & 5.0 & 7.1 & 5.8 & 5.9 & 4.8 & 5.2 & 4.9 \\
\hline Average nominal interest rate on forex debt (in percent) & 1.6 & 0.9 & 0.9 & 1.8 & 0.7 & 0.8 & 1.1 & 1.3 & 1.3 & 1.3 & 1.3 & 1.2 & 2.0 & 2.9 & 2.2 \\
\hline Average real interest rate on domestic debt (in percent) & -2.2 & -1.3 & -10.2 & 6.8 & 18.1 & 2.5 & 18.5 & 11.4 & 11.0 & 12.1 & 10.5 & 11.0 & 5.3 & 4.3 & 5.2 \\
\hline Real exchange rate depreciation (in percent, + indicates depreciation) & -6.3 & -15.0 & -22.0 & -9.4 & 7.8 & -4.0 & & $\ldots$ & & $\ldots$ & & $\ldots$ & $\ldots$ & & $\ldots$ \\
\hline Inflation rate (GDP deflator, in percent) & 11.2 & 11.9 & 22.1 & 4.4 & 14.3 & 19.3 & 2.8 & 7.3 & 3.9 & 2.4 & 2.0 & 6.3 & 1.7 & 2.0 & 1.8 \\
\hline Growth of real primary spending (deflated by GDP deflator, in percent) & 0.0 & 0.1 & 0.2 & 0.0 & 0.1 & 0.1 & -0.1 & 0.1 & 0.1 & 0.1 & 0.1 & 0.1 & 0.0 & 0.1 & 0.1 \\
\hline Grant element of new external borrowing (in percent) & $\ldots$ & & $\ldots$ & $\ldots$ & $\ldots$ & 46.7 & 40.6 & 43.3 & 41.9 & 39.4 & 38.2 & 41.7 & $\begin{array}{r}17.6 \\
\end{array}$ & 11.7 & $\ldots$ \\
\hline
\end{tabular}

Sources: Kyrgyz authorities; and Fund staff estimates and projections.

$1 /$ General government gross debt.

$3 /$ Gross financing need is defined as the primary deficit plus debt service plus the stock of short-term debt at the end of the last period.

4/ Revenues excluding grants.

$5 /$ Debt service is defined as the sum of interest and amortization of medium and long-term debt. 
Table 2a. Sensitivity Analysis for Key Indicators of Public Debt 2008-28

\begin{tabular}{|c|c|c|c|c|c|c|c|c|}
\hline & \multicolumn{8}{|c|}{ Projections } \\
\hline & 2008 & 2009 & 2010 & 2011 & 2012 & 2013 & 2018 & 2028 \\
\hline \multicolumn{9}{|l|}{ PV of Debt-to-GDP Ratio } \\
\hline Baseline & 35.9 & 32.6 & 31.2 & 28.9 & 27.4 & 26.5 & 33.3 & 41.5 \\
\hline \multicolumn{9}{|l|}{ A. Alternative scenarios } \\
\hline A1. Real GDP growth and primary balance are at historical averages & 35.9 & 35.4 & 37.7 & 38.8 & 41.2 & 43.7 & 64.1 & 100.6 \\
\hline A2. Primary balance is unchanged from 2008 & 35.9 & 32.0 & 30.3 & 27.9 & 26.2 & 25.1 & 29.0 & 35.0 \\
\hline A3. Permanently lower GDP growth $1 /$ & 35.9 & 32.8 & 32.0 & 30.5 & 30.0 & 30.4 & 48.5 & 97.7 \\
\hline \multicolumn{9}{|l|}{ B. Bound tests } \\
\hline B1. Real GDP growth is at historical average minus one standard deviation in $2009-2010$ & 35.9 & 34.3 & 37.1 & 36.9 & 37.5 & 38.9 & 58.1 & 86.3 \\
\hline B2. Primary balance is at historical average minus one standard deviation in $2009-2010$ & 35.9 & 39.0 & 43.9 & 41.2 & 39.1 & 37.8 & 43.5 & 47.9 \\
\hline B3. Combination of B1-B2 using one half standard deviation shocks & 35.9 & 37.7 & 42.4 & 41.0 & 40.3 & 40.3 & 53.2 & 69.8 \\
\hline B4. One-time 30 percent real depreciation in 2009 & 35.9 & 44.6 & 41.6 & 38.4 & 36.4 & 35.2 & 41.8 & 50.6 \\
\hline B5. 10 percent of GDP increase in other debt-creating flows in 2009 & 35.9 & 41.9 & 40.0 & 37.2 & 35.4 & 34.2 & 40.2 & 45.3 \\
\hline \multicolumn{9}{|c|}{ PV of Debt-to-Revenue Ratio 2/ } \\
\hline Baseline & 113.0 & 114.3 & 108.7 & 99.5 & 94.3 & 89.9 & 109.2 & 131.6 \\
\hline \multicolumn{9}{|l|}{ A. Alternative scenarios } \\
\hline A1. Real GDP growth and primary balance are at historical averages & 113.0 & 124.3 & 131.4 & 133.5 & 141.6 & 147.8 & 209.4 & 318.2 \\
\hline A2. Primary balance is unchanged from 2008 & 113.0 & 112.4 & 105.6 & 96.0 & 90.1 & 85.0 & 94.8 & 110.6 \\
\hline A3. Permanently lower GDP growth $1 /$ & 113.0 & 115.1 & 111.4 & 104.8 & 103.3 & 103.1 & 158.3 & 308.7 \\
\hline \multicolumn{9}{|l|}{ B. Bound tests } \\
\hline B1. Real GDP growth is at historical average minus one standard deviation in $2009-2010$ & 113.0 & 120.1 & 128.6 & 126.4 & 128.7 & 131.2 & 189.6 & 273.0 \\
\hline B2. Primary balance is at historical average minus one standard deviation in $2009-2010$ & 113.0 & 136.7 & 153.3 & 141.8 & 134.6 & 128.2 & 142.5 & 151.9 \\
\hline B3. Combination of B1-B2 using one half standard deviation shocks & 113.0 & 132.4 & 147.6 & 141.0 & 138.5 & 136.4 & 173.8 & 220.6 \\
\hline B4. One-time 30 percent real depreciation in 2009 & 113.0 & 156.5 & 145.0 & 132.2 & 125.4 & 119.4 & 137.0 & 160.4 \\
\hline B5. 10 percent of GDP increase in other debt-creating flows in 2009 & 113.0 & 147.1 & 139.5 & 128.3 & 122.0 & 115.9 & 131.6 & 143.6 \\
\hline
\end{tabular}

Debt Service-to-Revenue Ratio 2/

Baseline

$\begin{array}{llllllll}6.8 & 8.0 & 7.0 & 6.5 & 5.6 & 5.1 & 7.3 & 10.0\end{array}$

A. Alternative scenarios

A1. Real GDP growth and primary balance are at historical averages

A2. Primary balance is unchanged from 2008

A3. Permanently lower GDP growth $1 /$

$\begin{array}{rrrrrrrr}6.8 & 8.0 & 8.2 & 14.0 & 14.7 & 18.2 & 25.8 & 43.9 \\ 6.8 & 8.0 & 6.9 & 5.8 & 5.1 & 4.6 & 4.8 & 7.7\end{array}$

B. Bound tests

B1. Real GDP growth is at historical average minus one standard deviation in 2009-2010

B2. Primary balance is at historical average minus one standard deviation in 2009-2010

B3. Combination of B1-B2 using one half standard deviation shocks

B4. One-time 30 percent real depreciation in 2009

B5. 10 percent of GDP increase in other debt-creating flows in 2009

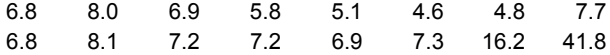

Sources: Kyrgyz authorities; and Fund staff estimates and projections.

1/ Assumes that real GDP growth is at baseline minus one standard deviation divided by the length of the projection period.

2/ Revenues are defined inclusive of grants.

$\begin{array}{rrrrrrrr}6.8 & 8.3 & 8.0 & 9.5 & 11.8 & 12.8 & 22.4 & 35.8 \\ 6.8 & 8.0 & 9.1 & 21.2 & 21.0 & 14.6 & 12.5 & 15.6 \\ 6.8 & 8.1 & 9.0 & 18.1 & 17.8 & 15.6 & 18.6 & 27.3 \\ 6.8 & 9.3 & 9.6 & 9.6 & 9.3 & 9.0 & 13.4 & 20.8 \\ 6.8 & 8.0 & 10.1 & 24.9 & 8.9 & 15.6 & 9.9 & 14.0\end{array}$


Table 3a. External Debt Sustainability Framework, Baseline Scenario, 2005-28 1/

(In percent of GDP, unless otherwise indicated)

\begin{tabular}{|c|c|c|c|c|c|c|c|c|c|c|c|c|c|c|c|}
\hline & \multicolumn{3}{|c|}{ Actual } & \multirow{2}{*}{$\begin{array}{l}\text { Historical } \\
\text { Average }\end{array}$} & \multirow{2}{*}{$\begin{array}{l}\text { Standard } \\
\text { Deviation }\end{array}$} & \multicolumn{6}{|c|}{ Projections } & \multirow[b]{2}{*}{$\begin{array}{l}2008-13 \\
\text { Average } \\
\end{array}$} & \multirow[b]{2}{*}{2018} & \multirow[b]{2}{*}{2028} & \multirow[b]{2}{*}{$\begin{array}{c}\begin{array}{c}2014-28 \\
\text { Average }\end{array} \\
\end{array}$} \\
\hline & 2005 & 2006 & 2007 & & & 2008 & 2009 & 2010 & 2011 & 2012 & 2013 & & & & \\
\hline External debt (nominal) 1/ & 85.5 & 77.7 & 61.1 & & & 49.3 & 48.6 & 45.0 & 42.6 & 39.0 & 36.4 & & 35.5 & 29.5 & \\
\hline o/w public and publicly guaranteed (PPG) & 78.0 & 69.8 & 55.5 & & & 44.4 & 43.8 & 41.1 & 39.6 & 36.7 & 34.7 & & 33.4 & 27.1 & \\
\hline Change in external debt & -9.6 & -7.8 & -16.6 & & & -11.9 & -0.7 & -3.6 & -2.4 & -3.6 & -2.6 & & -0.5 & -0.9 & \\
\hline Non-interest current account deficit & -4.3 & 2.4 & -0.5 & -0.1 & 4.6 & 6.0 & 7.9 & 5.6 & 4.4 & 3.2 & 2.8 & & 3.4 & 3.6 & 3.4 \\
\hline Deficit in balance of goods and services & 14.0 & 27.1 & 26.0 & & & 30.0 & 30.0 & 26.9 & 26.5 & 24.1 & 23.4 & & 24.7 & 21.4 & \\
\hline Exports & 42.8 & 52.3 & 59.9 & & & 65.3 & 59.3 & 59.5 & 60.5 & 62.4 & 63.6 & & 62.9 & 63.3 & \\
\hline Imports & 56.8 & 79.4 & 85.9 & & & 95.3 & 89.2 & 86.5 & 87.0 & 86.5 & 87.0 & & 87.6 & 84.7 & \\
\hline Net current transfers (negative = inflow) & -20.3 & -25.7 & -27.2 & -12.3 & 9.0 & -26.5 & -24.1 & -23.6 & -23.7 & -23.7 & -23.3 & & -22.5 & -19.0 & -21.4 \\
\hline Endogenous debt dynamics $2 /$ & -8.0 & -10.7 & -18.2 & & & -3.1 & -1.1 & -2.0 & -1.4 & -2.1 & -1.4 & & -0.7 & -0.4 & \\
\hline interest rate & 1.5 & 0.7 & 0.7 & & & 0.5 & 0.7 & 0.6 & 0.7 & 0.7 & 0.7 & & 0.9 & 1.1 & \\
\hline Contribution from real GDP grow & 0.1 & -2.3 & -4.8 & & & -3.6 & -1.7 & -2.6 & -2.1 & -2.8 & -2.1 & & -1.6 & -1.5 & \\
\hline Contribution from price and exchange rate changes & -9.6 & -9.1 & -14.1 & & & & & & & & & & & & \\
\hline Residual (3-4) $3 /$ & 4.4 & 6.9 & 7.7 & & & -9.1 & -2.1 & -2.5 & -1.0 & -0.3 & 0.1 & & 0.5 & -0.5 & \\
\hline $\mathrm{o} / \mathrm{w}$ exceptional financing & -1.8 & -0.2 & -0.1 & & & 0.0 & 0.0 & 0.0 & 0.0 & 0.0 & 0.0 & & 0.0 & 0.0 & \\
\hline PV of external debt $4 /$ & $\ldots$ & & 42.3 & & & 34.2 & 33.9 & 31.2 & 29.5 & 27.2 & 25.6 & & 27.8 & 25.9 & \\
\hline In percent of exports & $\ldots$ & $\ldots$ & 70.6 & & & 52.3 & 57.2 & 52.5 & 48.8 & 43.6 & 40.2 & & 44.3 & 40.9 & \\
\hline Total gross financing need (Billions of U.S. dollars) & -0.1 & 0.0 & -0.1 & & & 0.1 & 0.2 & 0.2 & 0.2 & 0.1 & 0.1 & & 0.3 & 0.8 & \\
\hline Non-interest current account deficit that stabilizes debt ratio & 5.4 & 10.2 & 16.1 & & & 17.8 & 8.6 & 9.2 & 6.8 & 6.8 & 5.4 & & 3.8 & 4.5 & \\
\hline \multicolumn{16}{|l|}{ Key macroeconomic assumptions } \\
\hline Real GDP growth (in percent) & -0.2 & 3.1 & 8.2 & 4.4 & 4.1 & 7.5 & 3.7 & 6.2 & 5.0 & 7.1 & 5.8 & 5.9 & 4.8 & 5.2 & 4.9 \\
\hline ar terms (change in percent) & 11.2 & 11.9 & 22.1 & 4.4 & 14.3 & 19.3 & 2.8 & 7.3 & 3.9 & 2.4 & 2.0 & 6.3 & 1.7 & 2.0 & 1.8 \\
\hline Effective interest rate (percent) $5 /$ & 1.7 & 0.9 & 1.2 & 2.3 & 1.4 & 1.0 & 1.4 & 1.5 & 1.6 & 1.7 & 1.8 & 1.5 & 2.6 & 3.7 & 3.0 \\
\hline Growth of exports of G\&S (US dollar terms, in percent) & 2.9 & 40.9 & 51.1 & 14.5 & 21.5 & 39.9 & -3.2 & 14.5 & 10.9 & 13.1 & 10.2 & 14.2 & 6.9 & 7.3 & 6.8 \\
\hline Growth of imports of G\&S (US dollar terms, in $p$ & 23.9 & 61.3 & 42.8 & 17.3 & 25.6 & 42.3 & -0.2 & 10.4 & 9.9 & 9.1 & 8.6 & 13.4 & 6.4 & 6.7 & 6.6 \\
\hline Grant element of new public sector borrowing & & & & & & 46.7 & 40.6 & 43.3 & 41.9 & 39.4 & 38.2 & 41.7 & 17.6 & 11.7 & 15.8 \\
\hline percent of GDP) & 23.7 & 25.6 & 28.5 & & & 29.1 & 25.9 & 26.7 & 27.3 & 27.4 & 27.9 & & 29.3 & 30.9 & 29.7 \\
\hline ions of US dollars) $6 /$ & 0.1 & 0.1 & 0.2 & & & 0.2 & 0.4 & 0.6 & 0.8 & 0.9 & 1. & & 1.9 & 4.8 & \\
\hline & 0.0 & 0.0 & 0.1 & & & 0.1 & 0.1 & 0.1 & 0.1 & 0.1 & 0.1 & & 0.1 & 0.1 & \\
\hline Concessional loans & 0.1 & 0.1 & 0.1 & & & 0.1 & 0.3 & 0. & 0.7 & 0.8 & 0.9 & & 1.8 & 4.7 & \\
\hline Grant-equivalent financing (in percent of GDP) $7 /$ & $\ldots$ & & $\ldots$ & & & 3.8 & 4.0 & 3.6 & 3.0 & 2.3 & 2.1 & & 1.7 & 1.0 & 1.5 \\
\hline Grant-equivalent financing (in percent of external financing) $7 /$ & $\ldots$ & $\cdots$ & $\ldots$ & & & 74.7 & 66.6 & 62.4 & 63.3 & 70.3 & 69.8 & & 42.0 & 30.1 & 37.8 \\
\hline \multicolumn{16}{|l|}{ Memorandu } \\
\hline Nominal GDP (Billions of US dollars) & 2.5 & 2.8 & 3.7 & & & 4.8 & 5.1 & 5.8 & 6.4 & 7.0 & 7.6 & & 10.2 & 20.2 & \\
\hline
\end{tabular}

Source: Staff simulations.

a de

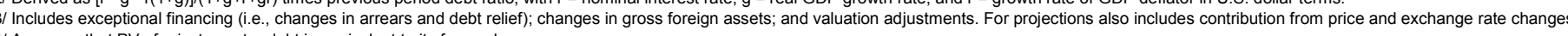
5/ Current-year interest payments divided by previous period debt value.

$6 /$ Defined as grants, concessional loans, and debt relief.

$7 /$ Grant-equivalent financing includes grants provided directly to the government and through new borrowing (difference between the face value and the PV of new debt). 
Table 3b. Sensitivity Analysis for Key Indicators of Public and Publicly Guaranteed External Debt, 2008-28 (In percent)

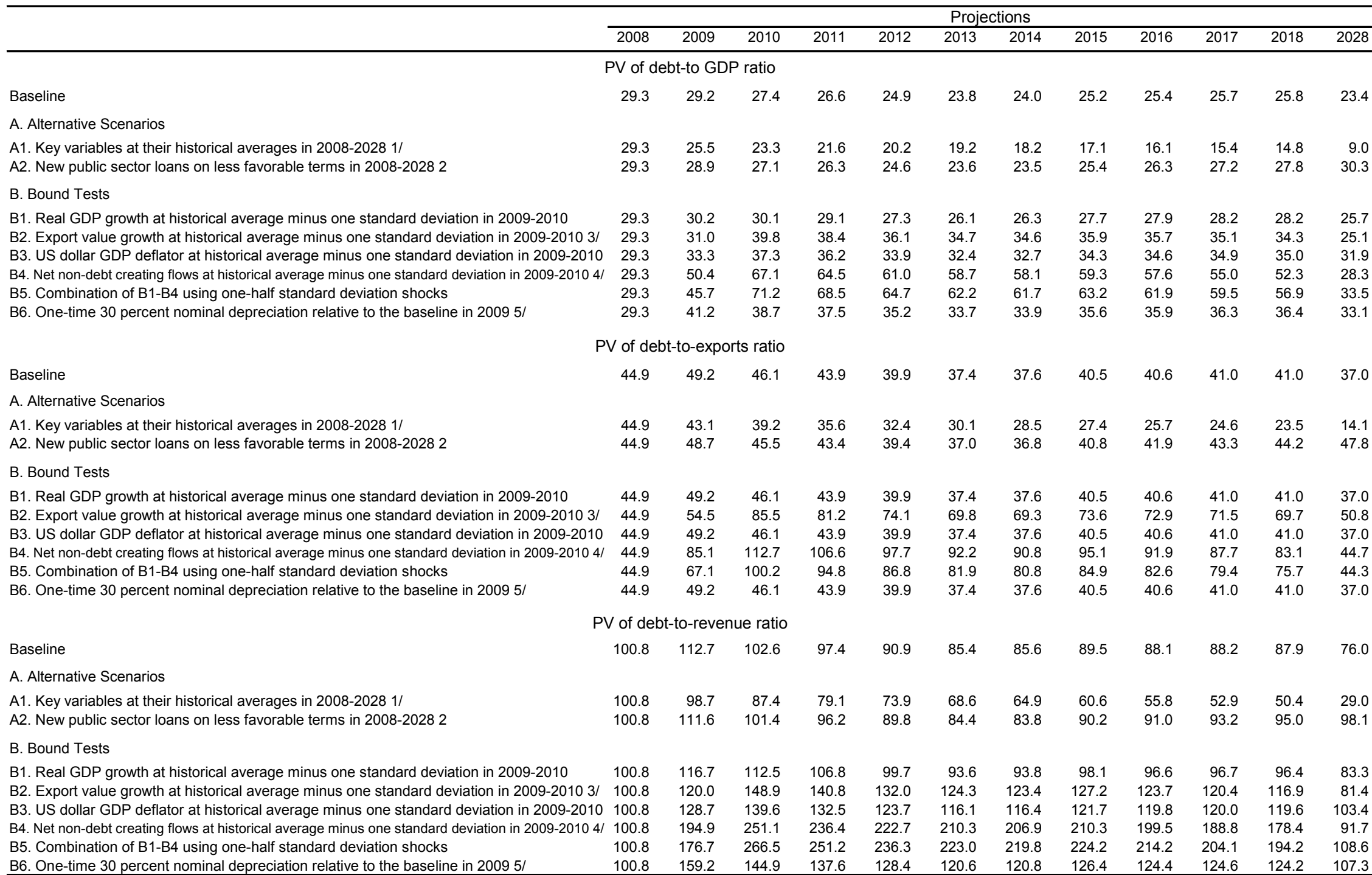

B6. One-time 30 percent nominal depreciation relative to the baseline in $20095 /$

\begin{tabular}{rr}
223.0 \\
120.6 & 120.8 \\
\hline
\end{tabular}

126.4

124.6 
Table 3b. Sensitivity Analysis for Key Indicators of Public and Publicly Guaranteed External Debt, 2008-28 (continued) (In percent)

\begin{tabular}{|c|c|c|c|c|c|c|c|c|c|c|c|c|}
\hline & \multicolumn{12}{|c|}{ Projections } \\
\hline & 2008 & 2009 & 2010 & 2011 & 2012 & 2013 & 2014 & 2015 & 2016 & 2017 & 2018 & 2028 \\
\hline \multicolumn{13}{|c|}{ Debt service-to-exports ratio } \\
\hline Baseline & 2.4 & 3.0 & 2.7 & 2.5 & 2.3 & 2.0 & 2.0 & 2.2 & 2.5 & 2.8 & 3.0 & 3.9 \\
\hline \multicolumn{13}{|l|}{ A. Alternative Scenarios } \\
\hline A1. Key variables at their historical averages in 2008-2028 1/ & 2.4 & 2.9 & 2.6 & 2.3 & 2.0 & 1.8 & 1.7 & 1.8 & 1.8 & 1.8 & 1.8 & 1.7 \\
\hline A2. New public sector loans on less favorable terms in 2008-2028 2 & 2.4 & 3.0 & 2.8 & 2.7 & 2.5 & 2.3 & 2.2 & 2.2 & 2.3 & 2.5 & 2.6 & 2.9 \\
\hline \multicolumn{13}{|l|}{ B. Bound Tests } \\
\hline B1. Real GDP growth at historical average minus one standard deviation in 2009-2010 & 2.4 & 3.0 & 2.7 & 2.5 & 2.3 & 2.0 & 2.0 & 2.2 & 2.5 & 2.8 & 3.0 & 3.9 \\
\hline B2. Export value growth at historical average minus one standard deviation in 2009-2010 3/ & 2.4 & 3.1 & 3.6 & 4.1 & 3.7 & 3.4 & 3.3 & 3.6 & 4.2 & 5.7 & 5.9 & 6.0 \\
\hline B3. US dollar GDP deflator at historical average minus one standard deviation in 2009-2010 & 2.4 & 3.0 & 2.7 & 2.5 & 2.3 & 2.0 & 2.0 & 2.2 & 2.5 & 2.8 & 3.0 & 3.9 \\
\hline B4. Net non-debt creating flows at historical average minus one standard deviation in 2009-2010 4 / & 2.4 & 3.0 & 3.9 & 4.8 & 4.3 & 3.9 & 3.8 & 4.1 & 6.1 & 8.0 & 8.0 & 6.5 \\
\hline B5. Combination of B1-B4 using one-half standard deviation shocks & 2.4 & 2.8 & 3.6 & 4.4 & 3.9 & 3.6 & 3.5 & 3.7 & 5.2 & 7.0 & 7.0 & 6.0 \\
\hline B6. One-time 30 percent nominal depreciation relative to the baseline in $20095 /$ & 2.4 & 3.0 & 2.7 & 2.5 & 2.3 & 2.0 & 2.0 & 2.2 & 2.5 & 2.8 & 3.0 & 3.9 \\
\hline \multicolumn{13}{|c|}{ Debt service-to-revenue ratio } \\
\hline Baseline & 5.4 & 6.8 & 6.1 & 5.7 & 5.1 & 4.6 & 4.5 & 4.9 & 5.5 & 6.0 & 6.5 & 7.9 \\
\hline \multicolumn{13}{|l|}{ A. Alternative Scenarios } \\
\hline A1. Key variables at their historical averages in 2008-2028 1/ & 5.4 & 6.7 & 5.8 & 5.2 & 4.6 & 4.1 & 3.9 & 4.0 & 3.8 & 3.8 & 3.9 & 3.5 \\
\hline A2. New public sector loans on less favorable terms in 2008-2028 2 & 5.4 & 6.8 & 6.2 & 6.0 & 5.7 & 5.2 & 4.9 & 4.9 & 5.1 & 5.3 & 5.6 & 6.0 \\
\hline \multicolumn{13}{|l|}{ B. Bound Tests } \\
\hline B1. Real GDP growth at historical average minus one standard deviation in 2009-2010 & 5.4 & 7.1 & 6.7 & 6.2 & 5.6 & 5.1 & 5.0 & 5.4 & 6.0 & 6.5 & 7.1 & 8.7 \\
\hline B2. Export value growth at historical average minus one standard deviation in 2009-2010 3/ & 5.4 & 6.8 & 6.3 & 7.2 & 6.6 & 6.0 & 5.8 & 6.2 & 7.1 & 9.5 & 9.8 & 9.6 \\
\hline B3. US dollar GDP deflator at historical average minus one standard deviation in 2009-2010 & 5.4 & 7.8 & 8.3 & 7.7 & 7.0 & 6.3 & 6.2 & 6.7 & 7.5 & 8.1 & 8.8 & 10.8 \\
\hline $\begin{array}{l}\text { B4. Net non-debt creating flows at historical average minus one standard deviation in 2009-2010 4/ } \\
\text { / }\end{array}$ & 5.4 & 6.8 & 8.7 & 10.6 & 9.8 & 8.9 & 8.7 & 9.0 & 13.3 & 17.3 & 17.1 & 13.3 \\
\hline B5. Combination of B1-B4 using one-half standard deviation shocks & 5.4 & 7.3 & 9.5 & 11.6 & 10.7 & 9.8 & 9.5 & 9.9 & 13.5 & 18.1 & 18.1 & 14.8 \\
\hline B6. One-time 30 percent nominal depreciation relative to the baseline in $20095 /$ & 5.4 & 9.6 & 8.6 & 8.0 & 7.3 & 6.5 & 6.4 & 6.9 & 7.8 & 8.4 & 9.1 & 11.2 \\
\hline Memorandum item: & & & & & & & & & & & & \\
\hline Grant element assumed on residual financing (i.e., financing required above baseline) 6/ & 18.0 & 18.0 & 18.0 & 18.0 & 18.0 & 18.0 & 18.0 & 18.0 & 18.0 & 18.0 & 18.0 & 18.0 \\
\hline
\end{tabular}

\section{Source: Staff projections and simulations.}

1/ Variables include real GDP growth, growth of GDP deflator (in U.S. dollar terms), non-interest current account in percent of GDP, and non-debt creating flows.

2/ Assumes that the interest rate on new borrowing is by 2 percentage points higher than in the baseline., while grace and maturity periods are the same as in the baseline.

3 / Exports values are assumed to remain permanently at the lower level, but the current account as a share of GDP is assumed to return to its baseline level after the shock (implicitly assuming an offsetting adjustment in import levels).

$4 /$ Includes official and private transfers and FDI.

$5 /$ Depreciation is defined as percentage decline in dollar/local currency rate, such that it never exceeds 100 percent.

6/ Applies to all stress scenarios except for A2 (less favorable financing) in which the terms on all new financing are as specified in footnote 2. 
Press Release No. 08/316

FOR IMMEDIATE RELEASE

December 10, 2008
International Monetary Fund

Washington, D.C. 20431 USA

\section{IMF Executive Board Approves US\$100 Million Exogenous Shocks Facility Arrangement for the Kyrgyz Republic}

The Executive Board of the International Monetary Fund (IMF) has approved an 18-month, SDR 66.6 million (about US\$100 million) arrangement under the Exogenous Shocks Facility (ESF) for the Kyrgyz Republic, to support the authorities in addressing several exogenous shocks, including the rise in commodity prices until mid-2008, a shortfall in hydropower, banking sector difficulties in neighboring Kazakhstan, and an earthquake in the Nura region.

The Executive Board's decision will enable the Kyrgyz Republic to draw an amount equivalent to SDR 16.65 million (about US\$25 million) from the IMF immediately.

In September 2008, the Executive Board approved modifications to the ESF which provided for faster and higher access, made the facility easier to use, and enhanced its flexibility. These modifications took effect in late November following the receipt of all the necessary legal consents.

Following the Executive Board's discussion, Mr. Murilo Portugal, Deputy Managing Director and Acting Chair, stated:

"The Kyrgyz economy has been adversely affected since late 2007 by several exogenous shocks that threaten to undermine macroeconomic stability, erode the gains made in poverty reduction, and create balance of payments difficulties. International food and fuel prices rose sharply until mid-2008, while low water levels are causing hydro-electric power shortages that necessitate an increase in imports of fuel and electricity. As a result, inflation has surged and the current account deficit has widened. Weakening global and regional growth in the wake of the international financial crisis, and spillovers from banking sector difficulties in neighboring countries that have reduced credit expansion in the Kyrgyz Republic, have led to a slowdown in the pace of economic growth. 
"The Kyrgyz authorities have adopted an economic program to address the consequences of these shocks. The program, which will be supported by an arrangement with the IMF under its Exogenous Shocks Facility, aims to reduce inflation, maintain an adequate level of foreign exchange reserves, sustain economic growth, and provide targeted protection to the poor.

"The program emphasizes the maintenance of cautious monetary and fiscal policies and greater exchange rate flexibility. Fiscal discipline will be maintained through cuts in nonpriority spending, tight control over the public sector wage bill, and a strengthening of public financial management. The banking system has been resilient so far to the global and regional financial shocks. The central bank will continue to monitor financial developments closely in order to assess vulnerabilities and mitigate risks, while strengthening banking supervision and regulation," Mr. Portugal said. 


\section{Recent Economic Developments}

Sound economic policies in the Kyrgyz Republic have contributed to strong economic performance. Economic growth accelerated to over 8 percent in 2007. Excluding gold production, it reached almost 9 percent. Inflation remained low until late 2007. Fiscal performance was also impressive, with revenues rising sharply. With firm fiscal policies, and aided by the economic recovery, a gradual appreciation of the som, and Paris Club support, the country was able to achieve a major improvement in debt indicators. Living standards improved as well and poverty rates fell from 54 percent in 2002 to 35 percent in 2007, while extreme poverty dropped from 23 percent to 7 percent.

However, since late 2007, the Kyrgyz economy has been hit by a number of exogenous and largely temporary shocks. International commodity prices continued to rise through much of 2008 , resulting in a surge in inflation and a widening current account deficit. A major shortfall in domestic hydro-power capacity due to low water levels is causing power shortages and necessitates the import of larger volumes of fuel and electricity, increasing the current account deficit further. Global and regional economic growth continues to fall in the wake of the international financial crisis and the Kyrgyz Republic's exports and inflows of workers remittances are expected to weaken, leading to a further widening of the current account deficit in 2009, despite the recent decline in commodity prices. Spillovers from banking sector difficulties in Kazakhstan have resulted in a sharp slowdown in credit expansion in the Kyrgyz Republic. As a result of the combined shocks, the pace of economic growth in the Kyrgyz Republic is slowing down as well this year and is expected to slow further in 2009.

\section{Program Summary}

The authorities' economic program for 2008-09 addresses the consequences of the exogenous shocks and aims to manage the effects on the Kyrgyz economy of the slowdown in regional growth and spillovers from the global financial crisis. The program's objectives are to reduce inflation, sustain economic growth, and protect the poor.

The program relies mainly on macroeconomic policies to achieve its goals, with policies continuing to walk a fine line between further reducing inflation and supporting growth. Monetary policy has been tightened substantially, reflected in a sharp increase in interest rates this summer, and aims to bring down inflation to close to 10 percent by end-2009. But as inflation falls, monetary policy could be eased gradually to aid the slowing economy. Fiscal policy will similarly aim to balance the need to help reduce inflation with the need to support growth and provide targeted protection to the poor. The program also includes a number of structural measures to support these policies, focusing on strengthening economic management. The authorities' economic program will support implementation of their Country Development Strategy (CDS). 
Kyrgyz Republic: Selected Economic Indicators, 2005-09

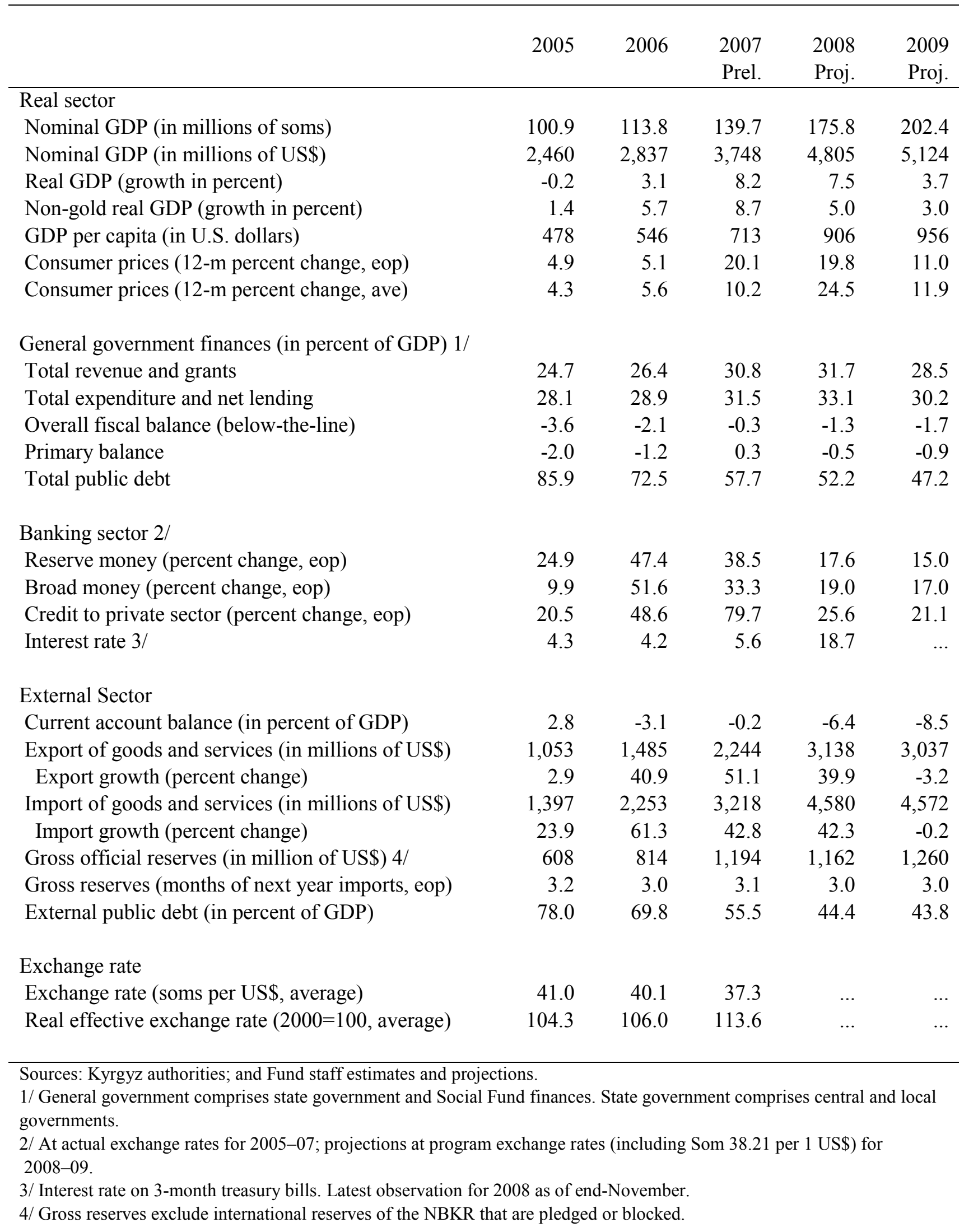




\section{Statement by Thomas Moser, Executive Director for the Kyrgyz Republic December 10, 2008}

1. My Kyrgyz authorities would like to thank the staff for the very constructive policy discussions and the continuous dialogue since the successful completion of the final PRGF review in May. Until early this year, macroeconomic performance in the Kyrgyz Republic was strong. Since then, however, a number of exogenous shocks threaten to undermine the country's hard-won macroeconomic stability. The authorities have decided to request access to Fund resources under the Exogenous Shocks Facility (ESF) rather than under a new 3-year PRGF arrangement, because the shocks are largely temporary in nature, creating a temporary balance of payments need. Moreover, given the size of these shocks, the authorities would like to focus their policy efforts on the immediate challenges ahead.

2. As a small and open economy, the Kyrgyz Republic is particularly vulnerable to external shocks. Apart from the commodity price shock, the slowdown in regional economic growth, and the spillovers from the global financial crisis, the low water level in the Toktogul reservoir impairs the country's hydropower capacity, making additional energy imports necessary. Overall, the current account deficit is projected to widen by more than 6 percent of GDP this year and another 2 percent of GDP next year before declining again. The requested Fund support would cover about half of the projected balance of payments needs, helping to smooth adjustment and maintain reserve coverage at 3 months of imports. The Kyrgyz authorities appreciate that the donor community has been responsive and increased their support, and they hope to mobilize further budget support.

3. The authorities' adjustment policies entail macroeconomic and structural measures. As to macroeconomic policies, the National Bank of the Kyrgyz Republic (NBKR) has tightened monetary policy significantly and will continue to allow the exchange rate to adjust. On the fiscal side, the Medium Term Fiscal Framework for 2009-2011 targets to limit the overall budget deficit to an annual average level of about 1.5 percent of GDP, by improving revenue collection and containing spending growth. Assistance to the poor is being increased through targeted benefits, and the value added tax rates on grain and flour have been temporarily reduced. The UN has recently issued an emergency appeal to allow its agencies to support the Government in addressing acute humanitarian needs over the coming winter months.

4. The NBKR and the Government are taking measures to ensure the stability of the Kyrgyz banking system, and the authorities are prepared to deal with further potential spillovers from the global financial crisis. The roles of the NBKR and the Ministry of Finance in the bank resolution process are being clarified (end-December structural benchmark), and banking supervision is further strengthened. The Kyrgyz Parliament recently approved the introduction of a deposit insurance scheme, and the authorities are considering to advance its implementation. The minimum capital requirement for existing 
commercial banks will be doubled by end- 2010 .

5. The Kyrgyz authorities continue to advance their structural reform agenda, and many of the structural measures that had been pending at the time of the last PRGF review have since been implemented. Particularly, the reduction in the retirement age approved in 2007 has been reversed, and the Parliament has approved the privatization of Ayul Bank. In the energy sector, tariff increases were implemented in July this year and further increases are scheduled for 2009. Substantial progress is also being made in improving the business environment; the 2008 World Bank Doing Business Survey ranks the Kyrgyz Republic as one of the top three reformers of the year.

6. The proposed economic program, together with financial support from the Fund and donors should help the Kyrgyz authorities to safeguard macroeconomic stability and continue to advance their reform agenda. The authorities stand ready to take additional measures if necessary, in close consultation with the Fund. 